\title{
ON THE SET OF PERIODS FOR $\sigma$ MAPS
}

\author{
M. CARME LESEDUARTE AND JAUME LLIBRE
}

\begin{abstract}
Let $\sigma$ be the topological graph shaped like the letter $\sigma$. We denote by 0 the unique branching point of $\sigma$, and by $\mathbf{O}$ and $I$ the closures of the components of $\sigma \backslash\{0\}$ homeomorphics to the circle and the interval, respectively. A continuous map from $\sigma$ into itself satisfying that $f$ has a fixed point in $\mathbf{O}$, or $f$ has a fixed point and $f(0) \in \mathbf{I}$ is called a $\sigma$ map. These are the continuous self-maps of $\sigma$ whose sets of periods can be studied without the notion of rotation interval. We characterize the sets of periods of all $\sigma$ maps.
\end{abstract}

\section{INTRODUCTION AND MAIN RESULTS}

Let $E$ be a topological space. Our goal in this work will be to describe the structure of the set of periods for some class of self-maps on $E$.

The set of natural numbers, real numbers and complex numbers will be denoted by $\mathbb{N}, \mathbb{R}$ and $\mathbb{C}$ respectively.

For a map $f: E \longrightarrow E$ we use the symbol $f^{n}$ to denote $f \circ f \circ \ldots \circ f(n \in \mathbb{N}$ times), $f^{0}$ denotes the identity map on $E$. Then, for a point $x \in E$ we define the orbit of $x$, denoted by $\operatorname{Orb}_{f}(x)$, as the set $\left\{f^{n}(x): n=0,1,2, \cdots\right\}$. We say that $x$ is a fixed point of $f$ if $f(x)=x$. We say that $x$ is a periodic point of $f$ of period $k \in \mathbb{N}$ (or $k$-periodic) if $f^{k}(x)=x$ and $f^{i}(x) \neq x$ for $1 \leq i<k$. In this case we say that the orbit of $x$ is a periodic orbit of period $k$. Note that if $x$ is a periodic point of period $k$, then $\operatorname{Orb}_{f}(x)$ has exactly $k$ elements, each of which is a periodic point of period $k$. We denote by $\operatorname{Per}(f)$ the set of periods of all periodic points of $f$.

From now on, the topological space $E$ will denote one of the following spaces:

$$
\begin{aligned}
\mathbf{I} & =\left\{(x, y) \in \mathbb{R}^{2}: 0 \leq x \leq 1 \text { and } y=0\right\}, \\
\mathbf{Y} & =\left\{z \in \mathbb{C}: z^{3} \in[0,1]\right\}, \\
\mathbf{O} & =\left\{(x, y) \in \mathbb{R}^{2}: x^{2}+(y+1)^{2}=1\right\}, \\
\boldsymbol{\sigma} & =\mathbf{I} \cup \mathbf{O} .
\end{aligned}
$$

The point $0 \in \mathbf{Y}$ or the $0=(0,0) \in \sigma$ are called branching points.

An interval map is a continuous self-map on the interval I. Similarly we define $\mathbf{Y}$ maps, circle maps and sigma maps.

Received by the editors May 20, 1994 and, in revised form, November 17, 1994.

1991 Mathematics Subject Classification. Primary 54H20.

Key words and phrases. Periodic orbit, set of periods, Sarkovskii theorem.

The authors have been partially supported by a DGICYT grant no. PB 93-0860. 
We remark that any interval map or any $\mathbf{Y}$ map always has a fixed point, but there are circle maps and sigma maps without fixed points.

Our objective in this section is to describe the structure of the set $\operatorname{Per}(f)$ for a class of sigma maps. To this end, we need to introduce some orderings in the set of natural numbers adding or removing some few elements.

The Sarkovskii ordering $>_{s}$ on the set $\mathbb{N}_{s}=\mathbb{N} \cup\left\{2^{\infty}\right\}$ is given by

$3>_{s} 5>_{s} 7>_{s} \cdots>_{s} 2 \cdot 3>_{s} 2 \cdot 5>_{s} 2 \cdot 7>_{s} \cdots>_{s} 2^{2} \cdot 3>_{s} 2^{2} \cdot 5>_{s} 2^{2} \cdot 7>_{s}$ $\cdots>_{s} 2^{n} \cdot 3>_{s} 2^{n} \cdot 5>_{s} 2^{n} \cdot 7>_{s} \cdots>_{s} 2^{\infty}>_{s} \cdots>_{s} 2^{n}>_{s} \cdots>_{s} 2^{4}>_{s} 2^{3}>_{s}$ $2^{2}>_{s} 2>_{s} 1$.

More precisely, if $k=k^{\prime} \cdot 2^{p}$ where $p \in \mathbb{N}$ and $k^{\prime}$ is odd, we have:

(i) $k>_{s} 2^{\infty}$ if $k^{\prime}>1$,

(ii) $2^{\infty}>_{s} k$ if $k^{\prime}=1$,

and if $n=n^{\prime} \cdot 2^{q}$ where $q \in \mathbb{N}$ and $n^{\prime}$ is odd, then we have $n>_{s} k$ if and only if one of the following cases occurs:

(iii) $k^{\prime}>1, n^{\prime}>1$ and $p>q$,

(iv) $k^{\prime}>n^{\prime}>1$ and $p=q$,

(v) $k^{\prime}=1$ and $n^{\prime}>1$

(vi) $k^{\prime}=1, n^{\prime}=1$ and $p<q$.

We shall use the symbols $\geq_{s},<_{s}$ and $\leq_{s}$ in the natural way. We have to include the symbol $2^{\infty}$ to ensure the existence of supremum of every subset with respect to the ordering $>_{s}$. For $n \in \mathbb{N}_{s}$ we denote $S(n)=\left\{k \in \mathbb{N}: n \geq_{s} k\right\}$. So $S\left(2^{\infty}\right)=\left\{2^{i}: i=0,1,2, \cdots\right\}$.

Now we state the Sarkovskii Theorem, which is proved in [Sa] (see also [St], [BGMY] and [ALM2]).

Theorem 1.1 (Interval Theorem).

(a) If $f$ is an interval map, then $\operatorname{Per}(f)=S(n)$ for some $n \in \mathbb{N}_{s}$.

(b) If $n \in \mathbb{N}_{s}$, then there exists an interval map $f$ such that $\operatorname{Per}(f)=S(n)$.

If we want to get a similar result for the $Y$ space, we need two new orderings. The green ordering $>_{g}$ on $\mathbb{N} \backslash\{2\}$ is given by

$5>_{g} 8>_{g} 4>_{g} 11>_{g} 14>_{g} 7>_{g} 17>_{g} 20>_{g} 10>_{g} \cdots>_{g} 3 \cdot 3>_{g} 3 \cdot 5>_{g}$ $3 \cdot 7>_{g} \cdots>_{g} 3 \cdot 2 \cdot 3>_{g} 3 \cdot 2 \cdot 5>_{g} 3 \cdot 2 \cdot 7>_{g} \cdots>_{g} 3 \cdot 2^{2} \cdot 3>_{g} 3 \cdot 2^{2} \cdot 5>_{g}$ $3 \cdot 2^{2} \cdot 7>_{g} \cdots>_{g} 3 \cdot 2^{3}>_{g} 3 \cdot 2^{2}>_{g} 3 \cdot 2>_{g} 3 \cdot 1>_{g} 1$.

The first part of this ordering can be rewittren as

$6-1>_{g} 6+2>_{g} 3+1>_{g} 2 \cdot 6-1>_{g} 2 \cdot 6+2>_{g} 2 \cdot 3+1>_{g} 3 \cdot 6-1>_{g}$ $3 \cdot 6+2>_{g} 3 \cdot 3+1>_{g} \cdots$.

To be more precise, denote by the symbol $\equiv$ congruences modulus 3 . We have $k>_{g} n$ for $k, n \in \mathbb{N} \backslash\{2\}$ if and only if $n>1$ and one of the following cases occurs:

(i) $k \not \equiv 0, n \not \equiv 0, k \equiv n, k>n$,

(ii) $k \equiv 1, n \equiv 2,2 k \geq n$,

(iii) $k \equiv 2, n \equiv 1, k>2 n$,

(iv) $k \equiv 0, n \neq 0$,

(v) $k \equiv 0, n \equiv 0, k / 3>_{s} n / 3$,

(vi) $k=1$.

The red ordering $>_{r}$ on $\mathbb{N} \backslash\{2,4\}$ is given by 
$7>_{r} 10>_{r} 5>_{r} 13>_{r} 16>_{r} 8>_{r} 19>_{r} 22>_{r} 11>_{r} \cdots>_{r} 3 \cdot 3>_{r} 3 \cdot 5>_{r}$ $3 \cdot 7>_{r} \cdots>_{r} 3 \cdot 2 \cdot 3>_{r} 3 \cdot 2 \cdot 5>_{r} 3 \cdot 2 \cdot 7>_{r} \cdots>_{r} 3 \cdot 2^{2} \cdot 3>_{r} 3 \cdot 2^{2} \cdot 5>_{r}$ $3 \cdot 2^{2} \cdot 7>_{r} \cdots>_{r} 3 \cdot 2^{3}>_{r} 3 \cdot 2^{2}>_{r} 3 \cdot 2>_{r} 3 \cdot 1>_{r} 1$.

The first part of this ordering can be rewritten as

$6+1>_{r} 6+4>_{r} 3+2>_{r} 2 \cdot 6+1>_{r} 2 \cdot 6+4>_{r} 2 \cdot 3+2>_{r} 3 \cdot 6+1>_{r}$ $3 \cdot 6+4>_{r} 3 \cdot 3+2>_{r} \cdots$

We have $k>_{r} n$ for $k, n \in \mathbb{N} \backslash\{2,4\}$ if and only if $n>1$ and one of the following cases occurs:

(i) $k \not \equiv 0, n \not \equiv 0, k \equiv n, k>n$,

(ii) $k \equiv 2, n \equiv 1,2 k \geq n$,

(iii) $k \equiv 1, n \equiv 2, k>2 n$,

(iv) $k \equiv 0, n \neq 0$,

(v) $k \equiv 0, n \equiv 0, k / 3>_{s} n / 3$,

(vi) $k=1$.

For $n \in \mathbb{N} \backslash\{2\}$ denote $G(n)=\left\{k \in \mathbb{N}: n \geq_{g} k\right\}$, for $n \in \mathbb{N} \backslash\{2,4\}$ denote $R(n)=\left\{k \in \mathbb{N}: n \geq_{r} k\right\}$ and additionally $G\left(3 \cdot 2^{\infty}\right)=R\left(3 \cdot 2^{\infty}\right)=\{1\} \cup\{3 n: n \in$ $\left.S\left(2^{\infty}\right)\right\}$. We also denote $\mathbb{N}_{g}=(\mathbb{N} \backslash\{2\}) \cup\left\{3 \cdot 2^{\infty}\right\}$ and $\mathbb{N}_{r}=(\mathbb{N} \backslash\{2,4\}) \cup\left\{3 \cdot 2^{\infty}\right\}$.

The next theorem is due to Alsedà, Llibre and Misiurewicz [ALM1] for $Y$ maps with the branching point fixed and to Baldwin for arbitrary $\mathbf{Y}$ maps [Ba].

Theorem 1.2 (Y Theorem).

(a) If $f$ is an $\mathbf{Y}$ map, then $\operatorname{Per}(f)=S\left(n_{s}\right) \cup G\left(n_{g}\right) \cup R\left(n_{r}\right)$ for some $n_{s} \in \mathbb{N}_{s}, n_{g} \in \mathbb{N}_{g}$ and $n_{r} \in \mathbb{N}_{r}$.

(b) If $n_{s} \in \mathbb{N}_{s}, n_{g} \in \mathbb{N}_{g}$ and $n_{r} \in \mathbb{N}_{r}$, then there exists an $\mathbf{Y}$ map $f$ having the branching point fixed such that $\operatorname{Per}(f)=S\left(n_{s}\right) \cup G\left(n_{g}\right) \cup R\left(n_{r}\right)$.

We define the Block ordering $>_{b}$ on $\mathbb{N}_{b}=\mathbb{N} \backslash\{1\}$ as the converse of the natural ordering on $\mathbb{N}$; i.e. $2>_{b} 3>_{b} 4>_{b} \cdots$. For $n \in \mathbb{N}_{b}$, we denote $B(n)=\left\{k \in \mathbb{N}: n \geq_{b} k\right\} \cup\{1\}$. Sharkovskii's Theorem has been generalized by Block to the circle maps having a fixed point in [BI].

Theorem 1.3 (Circle Theorem).

(a) If $f$ is a circle map having a fuxed point, then $\operatorname{Per}(f)=S\left(n_{s}\right) \cup B\left(n_{b}\right)$ for some $n_{s} \in \mathbb{N}_{s}$ and $n_{b} \in \mathbb{N}_{b}$.

(b) If $n_{s} \in \mathbb{N}_{s}$ and $n_{b} \in \mathbb{N}_{b}$, then there exists a circle map $f$ having a fixed point such that $\operatorname{Per}(f)=S\left(n_{s}\right) \cup B\left(n_{b}\right)$.

The study of the set of periods for circle maps without fixed points is more difficult, and needs the notion of rotation interval. Here we do not consider maps without fixed points, for more details see [ALM2].

In this paper we extend the previous three theorems to a class of sigma maps. As for circle maps if we want to avoid in a first study of the sigma maps the problems related with the computation of the set of periods from the rotation interval, we must add some additional assumptions to the continuity of the map. While for circle maps it is sufficient to add the assumption that they have a fixed point, this is not the case for sigma maps. More concretely, in order to avoid the rotation interval associated to a sigma map in the computation of its set of periods, we must restrict our attention to the subclass of sigma maps $f$ such that $f$ has a fixed point and $f(0) \in \mathbf{I}$, or $f$ has a fixed point in $\mathbf{0}$. In what follows such sigma maps will be denoted $\sigma$ maps. We remark that our 


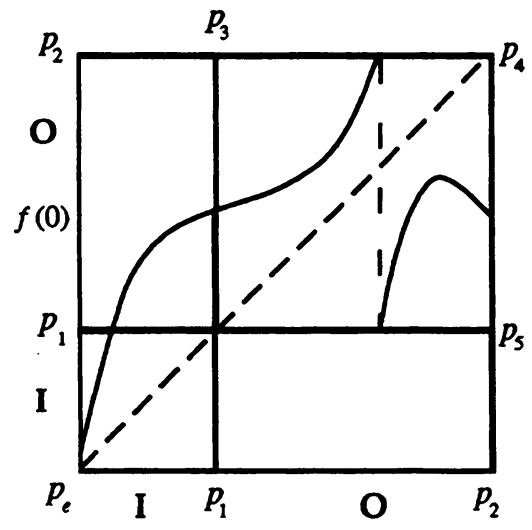

FIGURE 1.1. This sigma map $f$ satisfies that $\operatorname{Per}(f)=$ $\operatorname{Per}\left(\left.f\right|_{0}\right) \cup\{1\}$, and $\left.f\right|_{0}$ is a circle map of degree 1 without fixed points (see [ALM2].

$\sigma$ maps are the sigma maps such that $f(0) \in \mathbf{I}$ or $f$ has a fixed point in $\mathbf{O}$, because in the first case $f$ always has a fixed point in $\mathbf{I}$. In short the unique sigma maps having a fixed point that are no $\sigma$ maps satisfy that the fixed point do not belong to $\mathbf{O}$ and $f(0) \notin \mathbf{I}$; such maps need the rotation interval for describing the set of periods, see Figure 1.1 and [ALM2].

The graph of a sigma map $f$ is the subset $\{(x, f(x)): x \in \sigma\}$ of the cartesian product $\sigma \times \sigma$, and it can be represented as in Figure 1.1. More precisely, if in the closed square $\left[p_{e}, p_{2}\right] \times\left[p_{e}, p_{2}\right]$ of Figure 1.1 we identify the vertical straight lines $p_{1} p_{3}$ and $p_{2} p_{4}$, and the horizontal straight lines $p_{1} p_{5}$ and $p_{2} p_{4}$, we get the space $\sigma \times \sigma$. The segment $p_{e} p_{1}$ represents $I$, and the segment $p_{1} p_{2}$ with the points $p_{1}$ and $p_{2}$ identified to the branching point 0 represents $\mathbf{O}$. Roughly speaking we think in the graph of a sigma map like the graph of an interval map from $\left[p_{e}, p_{2}\right]$ into itself with the above identifications.

Our main result is the following one.

Theorem 1.4 ( $\sigma$ Theorem).

(a) If $f$ is a $\sigma$ map, then $\operatorname{Per}(f)=S\left(n_{s}\right) \cup G\left(n_{g}\right) \cup R\left(n_{r}\right) \cup B\left(n_{b}\right)$ for some $n_{s} \in \mathbb{N}_{s}, n_{g} \in \mathbb{N}_{g}, n_{r} \in \mathbb{N}_{r}$ and $n_{b} \in \mathbb{N}_{b}$.

(b) If $n_{s} \in \mathbb{N}_{s}, n_{g} \in \mathbb{N}_{g}, n_{r} \in \mathbb{N}_{r}$ and $n_{b} \in \mathbb{N}_{b}$, then there exists a $\sigma$ map $f$ having the branching point fixed such that $\operatorname{Per}(f)=S\left(n_{s}\right) \cup G\left(n_{g}\right) \cup$ $R\left(n_{r}\right) \cup B\left(n_{b}\right)$.

Theorem 1.4 when the branching point is fixed was proved simultaneously and in a different way by Llibre, Paraños and Rodriguez see [LPR]. As far as we know Theorem 1.4 is the first result on the characterization of the set of periods of continuous self-maps on topological graphs which are not trees without having fixed all the branching points.

\section{INTERVALS AND BASIC INTERVALS}

A closed (respectively open, half-open or half-closed) interval $J$ of $E$ is a subset of $E$ homeomorphic to the closed interval $[0,1]$ (resp. $(0,1)$, $[0,1))$. Notice that an interval cannot be a single point. 
Let $J$ be a closed interval of $E$, and let $h:[0,1] \longrightarrow J$ be a homeomorphism. Then $h(0)=a$ and $h(1)=b$ are called the endpoints of $J$. If either $E \in\{\mathbf{I}, \mathbf{Y}\}$ or $E=\boldsymbol{\sigma}$ and $a, b \in \mathbf{I}$, then $J$ will be denoted by $[a, b]$ or $[b, a]$. If $E=\mathbf{O}$ and $a, b \in \mathbf{O}$, then we write $[a, b]$ to denote the closed interval from $a$ counterclockwise to $b$. If $E=\boldsymbol{\sigma}, a \in \mathbf{O}$ and $b \in \mathbf{I}$, then $[a, b]$ will denote $[a, 0] \cup[0, b]$ or $[a, 0] \cup[b, 0]$, and $[b, a]$ will denote $[b, 0] \cup[0, a]$ or $[0, b] \cup[0, a]$.

Notice that it is possible that two different intervals of $\mathbf{O}$ or $\sigma$ have the same endpoints. But two different points of $I$ or $\mathbf{Y}$ always determine a unique closed interval.

Now we define an special class of subintervals of $E$. Let $Q=\left\{q_{1}, q_{2}, \cdots\right.$, $\left.q_{n}\right\}$ be a finit subset of $\sigma$. For each pair $q_{i}, q_{j}$ such that $q_{i} \neq q_{j}$ we say that the interval $\left[q_{i}, q_{j}\right]$ (respectively $\left[q_{j}, q_{i}\right]$ ) is basic if and only if $\left(q_{i}, q_{j}\right) \cap Q=\varnothing$ (respectively $\left.\left(q_{j}, q_{i}\right) \cap Q=\varnothing\right)$. The set of all these basic intervals is called the set of basic intervals associated to $Q$.

Assume that $E=\sigma, 0 \notin Q, Q \cap \mathbf{I} \neq \varnothing$ and $Q \cap \mathbf{O} \neq \varnothing$. Then we consider $Q^{\prime}=Q \cup\{0\}$. Clearly there are exactly three basic intervals $A, B$ and $C$ associated to $Q^{\prime}$ with 0 as endpoint. Without loss of generality we can assume that $A \subset \mathrm{I}$. The endpoint of $A, B$ and $C$ different from 0 is denoted by $x_{a}, x_{b}$ and $x_{c}$ respectively. We define $L_{1}, L_{2}, L_{3}$ as the three basic intervals associated to $Q$ containing 0 and such that $L_{1}=A \cup B, L_{2}=A \cup C, L_{3}=$ $B \cup C$. Notice that $L_{3}$ is an interval if and only if $x_{b} \neq x_{c}$. In what follows, the intervals $A, B, C$ and the endpoints $x_{a}, x_{b}, x_{c}$ are called the intervals $A, B, C$ and the endpoints $x_{a}, x_{b}, x_{c}$ associated to $Q^{\prime}$; the intervals $L_{1}, L_{2}$ and $L_{3}$ are called the basic intervals $L_{1}, L_{2}$ and $L_{3}$ associated to $Q$.

\section{LOOPS AND $f$-GRAPHS}

Let $f: E \rightarrow E$ be an $E$ map. If $K$ and $J$ are intervals of $E$, then we say that $K f$-covers $J$ or $K \rightarrow J$ (or $J \leftarrow K$ ), if there is a closed subinterval $M$ of $K$ such that $f(M)=J$. If $K$ does not $f$-cover $J$ we write $K \nrightarrow J$.

A path of length $m$ is any sequence $J_{0} \rightarrow J_{1} \rightarrow \cdots \rightarrow J_{m-1} \rightarrow J_{m}$, where $J_{0}, J_{1}, \ldots, J_{m}$ are closed subintervals of $E$ (in general, basic intervals). Furthermore, if $J_{0}=J_{m}$, then this path is called a loop of length $m$. Such a loop will be called non-repetitive if there is no integer $i, 0<i<m$, such that $i$ divides $m$ and $J_{j+i}=J_{j}$ for all $j, 0 \leq j \leq m-i$. This means that the loop is not a repetition of a shorter loop.

Let $Q$ be a finite subset of $E$. An $f$-graph of $Q$ is a graph with the basic intervals as vertices, and such that if $K$ and $J$ are basic intervals and $K f$ covers $J$, then there is an arrow from $K$ to $J$. Note that the $f$-graph of $Q$ is unique up to labeling of the basic intervals. Hence from now on we shall talk about the $f$-graph of $Q$ (or just the $f$-graph for short).

Lemma 3.1. Let $f$ be an $E$-map and let $K, J, L$ be closed subintervals of $E$. If $L \subset J$ and $K f$-covers $J$, then $K f$-covers $L$.

Proof. By hypotheses, there exists an interval $M \subset K$ such that $f(M)=J$. First we suppose that $E=\mathrm{I}$. Let $L=[c, d]$. Then there are $a, b \in M$ such that $f(a)=c$ and $f(b)=d$. We can assume that $a<b$. Then set $e=\sup \{x \in[a, b]: f(x)=f(a)\}$ and $l=\inf \{x \in[e, b]: f(x)=f(b)\}$. Clearly $f([e, l])=L$. So $K \rightarrow L$. 
Finally assume that $E \neq \mathbf{I}$. There exist homeomorphisms $h_{M}: M \longrightarrow \mathbf{I} \equiv$ $[0,1]$ and $h_{J}: J \longrightarrow I$. Note that $g=\left.h_{J} \circ f\right|_{M} \circ h_{M}^{-1}$ is an interval map. Since $g(\mathbf{I})=\mathbf{I}, \mathbf{I} f$-covers $\mathbf{I}$. By the first part of this proof, I $g$-covers the interval $h_{J}(L) \subset \mathbf{I}$. Then there exists a closed subinterval $N$ of I such that $g(N)=h_{J}(L)$. Therefore $h_{M}^{-1}(N)$ is a closed subinterval of $M$ and $f\left(h_{M}^{-1}(N)\right)=h_{J}^{-1} \circ g \circ h_{M}\left(h_{M}^{-1}(N)\right)=L$. So $K \rightarrow L$.

Lemma 3.2. Let $f$ be an $E$ map and let $J$ be a subinterval of $E$ such that $J$ $f$-covers $J$. Then $f$ has a fixed point in $J$.

Proof. First we consider the case $E=I$. Set $J=[a, b]$. Then there are points $c, d \in J$ such that $f(c)=a$ and $f(d)=b$. We obtain $f(c)-c \leq 0$ and $f(d)-d \geq 0$. Then by continuity of $f$, there is a point $x$ between $c$ and $d$ such that $f(x)-x=0$; i.e. $f(x)=x$.

Finally suppose that $E \neq I$. Since $J \rightarrow J$, there is a closed interval $L$ of $J$ such that $f(L)=J$. Then there exists a homeomorphism $h: J \longrightarrow \mathbf{I} \equiv[0,1]$ and we can consider an interval map $g$ defined as $g=\left.h \circ f\right|_{L} \circ h^{-1}$. Since $g(\mathbf{I})=\mathbf{I}$, by the first part of this proof there exists $y \in \mathbf{I}$ such that $g(y)=y$. Consequently for $x=h^{-1}(y) \in L$, we have $f(x)=h^{-1} \circ g \circ h(x)=h^{-1} \circ g(y)=$ $h^{-1}(y)=x$, and we are done.

Lemma 3.3. Let $f$ be an $E$ map and let $J_{0}, J_{1}, \cdots, J_{n-1}$ be closed subintervals of $E$ such that $J_{i} \rightarrow J_{i+1}$ for $i=0,1, \cdots, n-2$ and $J_{n-1} \rightarrow J_{0}$. Then there exists a fixed point $x$ of $f^{n}$ in $J_{0}$ such that $f^{i}(x) \in J_{i}$ for $i=$ $1,2, \cdots, n-1$.

Proof. We shall use backward induction. Let $K_{n-1} \subset J_{n-1}$ be a closed interval such that $f\left(K_{n-1}\right)=J_{0}$, and suppose we have constructed $K_{i} \subset J_{i}$ for some $i>0, i \leq n-1$ such that $f\left(K_{i}\right)=K_{i+1}$ if $i<n-1$ and $f\left(K_{i}\right)=J_{0}$ if $i=n-1$. Then, by Lemma 3.1, $J_{i-1} f$-covers $K_{i}$ and therefore there exists an interval $K_{i-1} \subset J_{i-1}$ such that $f\left(K_{i-1}\right)=K_{i}$. Let $\mathrm{g}$ be as follows: $g=\left.\left.\left.f\right|_{K_{n-1}} \circ \cdots \circ f\right|_{K_{1}} \circ f\right|_{K_{0}}$. Then $K_{0} \subset J_{0}$ and $g\left(K_{0}\right)=J_{0}$. Consequently $f^{n}\left(K_{0}\right)=J_{0}$. By continuity of $f^{n}$ and Lemma $3.2 f^{n}$ has a fixed point $x \in K_{0} \subset J_{0}$, such that $f^{i}(x) \in K_{i} \subset J_{i}$ for $i=1,2, \cdots, n-1$.

\section{Basic assumptions: $f, P$ and $k$}

Let $f$ be a $\sigma$ map. Notice that for proving Theorem 1.4(a) for $f$ it is suffcient to show that if $k \in \operatorname{Per}(f)$, then at least one of the sets $S(k), G(k), R(k)$ or $B(k)$ is contained in $\operatorname{Per}(f)$. So in what follows we fix the $\sigma$ map $f$ and a $k \in \operatorname{Per}(f)$ and we will prove the claim: at least one of the sets $S(k), G(k), R(k)$ or $B(k)$ is contained in $\operatorname{Per}(f)$. This fixed $\sigma$ map will be called the standard $\sigma$ map.

Since a $\sigma$ map have fixed points, we can assume that $k>1$. Furthermore, since $S(2)=\{1,2\}$ and $G(3)=R(3)=\{1,3\}$ we can assume that $k>3$. Due to the fact that $k \in \operatorname{Per}(f)$, there exists a periodic orbit $P=\left\{x_{1}, \cdots, x_{k}\right\}$ of period $k$ for $f$. Also in what follows $P$ always will denote a periodic orbit of period $k$ for $f$.

\section{Reduction to the Case $P \cap \mathbf{O} \backslash\{0\} \neq \varnothing$ aNd $P \cap \mathbf{I} \backslash\{0\} \neq \varnothing$}

The next proposition shows that if $P$ is contained either in $O$ or in $I$, then the claim of Section 4 follows. 
Proposition 5.1. Let $f$ be the standard $\sigma$ map. If either $P \subset \mathbf{O}$ or $P \subset I$, then $S(k)$ or $B(k)$ is contained in $\operatorname{Per}(f)$.

Proof. First assume that $P \subset \mathbf{O}$. We define $g: \mathbf{O} \longrightarrow \mathbf{O}$ by $g(x)=f(x)$ if $f(x) \in \mathbf{O}$, and $g(x)=0$ otherwise. Then $g$ is continuous and $P$ is a periodic orbit of period $k$ for $g$. Furthermore $g$ has a fixed point, because if $f(0) \in \mathbf{I}$, then $g(0)=0$; otherwise $f$ has a fixed point $z$ in $\mathbf{O}$ and so $g(z)=z$. From the Circle Theorem it follows that $\operatorname{Per}(g)=S\left(n_{s}\right) \cup B\left(n_{b}\right)$ for some $n_{s} \in \mathbb{N}_{s}, n_{b} \in \mathbb{N}_{b}$. Clearly $\operatorname{Per}(g) \subset \operatorname{Per}(f)$. Since $k \in \operatorname{Per}(g)$, we get that $S(k)$ or $B(k)$ is contained in $\operatorname{Per}(g)$. Consequently $S(k)$ or $B(k)$ is contained in $\operatorname{Per}(f)$.

Now suppose that $P \subset \mathbf{I}$. We define $g: \mathbf{I} \longrightarrow \mathbf{I}$ by $g(x)=f(x)$ if $f(x) \in \mathbf{I}$, and $g(x)=0$ otherwise. Then $g$ is an interval map. Furthermore $P$ is a periodic orbit of period $k$ for $g$. By the Interval Theorem $\operatorname{Per}(g)=S\left(n_{s}\right)$ for some $n_{s} \in \mathbb{N}_{s}$. Clearly $\operatorname{Per}(g) \subset \operatorname{Per}(f)$. Since $k \in \operatorname{Per}(g)$, we get $S(k) \subset \operatorname{Per}(g)$. Therefore $S(k) \subset \operatorname{Per}(f)$.

From now on we can assume that the periodic orbit $P$ has points in $\mathbf{O} \backslash\{0\}$ and points in $\mathbf{I} \backslash\{0\}$.

It is important to remark that Proposition 5.1 is not true if $P \subset \mathbf{O}, f(0) \in \mathbf{O}$ and $f$ has no fixed points in $\mathbf{O}$. This is due to the fact that in such a case the circle map $g: \mathbf{O} \longrightarrow \mathbf{O}$ defined in the first part of the proof of Proposition 5.1 has no fixed points, and consequently its set of periods depends on the rotation interval of $g$ (see [ALM2]). Since in this paper we do not deal with the rotation interval, this fact is the main reason which forces us to restrict our attention to the class of $\sigma$ maps instead of the class of all sigma maps.

\section{Reduction to THE CASE THE ENDPOINT OF $\boldsymbol{\sigma}$ Belongs to $\boldsymbol{P}$}

Denote by $q_{e}$ the endpoint of $\sigma$, and let $p_{e} \in P \cap \mathbf{I}$ be such that $\left(q_{e}, p_{e}\right)$ $\cap P=\varnothing$. Notice that such a $p_{e}$ exists in virtue of Section 5 .

Lemma 6.1. Let $f$ be the standard $\sigma$ map. Suppose that $f(0) \in \mathbf{I} \backslash\{0\}$. Then there exists a fixed point $z$ of $f$ such that $z \in\left[p_{e}, 0\right] \subset \mathbf{I}$.

Proof. Since $\left(q_{e}, p_{e}\right) \cap P=\varnothing, p_{e} \in P \cap I$ and $P$ is a periodic orbit, we have that $f\left(p_{e}\right) \notin\left[q_{e}, p_{e}\right]$. Moreover $f(0) \in \mathbf{I}$. Hence by the continuity of $f$, the subinterval $\left[p_{e}, 0\right] \subset \mathrm{I}$ contains a fixed point of $f$.

In a similar way as in Section 4 where we have fixed $f, P$ and $k$, now we shall fix a fixed point of $f$.

If $f(0)=0$ then let $x_{0}=0$.

Suppose that $f(0) \in \mathbf{O} \backslash\{0\}$. Then by the definition of $\sigma$ map, $f$ has fixed points in $\mathbf{O}$. We consider the following statements:

(a) $[0, f(0)] \cap P=\varnothing$ and the subinterval $[0, f(0)]$ contains fixed points of $f$.

(b) There is a fixed point $z \in \mathbf{O}$ of $f$ such that $[0, z] \cap P=\varnothing$ and $(0, z)$ does not contain fixed points of $f$.

If statement (a) is satisfied, then let $x_{0} \in[0, f(0)]$ be such that $\left[0, x_{0}\right] \subset$ $[0, f(0)]$ and $\left[0, x_{0}\right)$ does not contain fixed points. Suppose that $(a)$ is not satisfied. If statement (b) holds, let $x_{0}=z$ satisfying (b); otherwise let $x_{0}$ be an arbitrary fixed point of $f$ in $\mathbf{O}$. 
Suppose that $f(0) \in \mathbf{I} \backslash\{0\}$. From Lemma 6.1 there is a fixed point $z$ of $f$ such that $z \in\left[p_{e}, 0\right]$. Then let $x_{0} \in \mathbf{I}$ be the fixed point of $f$ such that $\left[p_{e}, x_{0}\right)$ does not contain fixed points.

Remark 6.2. In what follows $x_{0}$ always will be a fixed point of $f$ satisfying the above assumptions. Moreover we note that if $f(0) \neq 0$, then we have either $\left\{f(0), x_{0}\right\} \subset \mathbf{I}$ or $\left\{f(0), x_{0}\right\} \subset \mathbf{O}$.

Proposition 6.3. Let $f$ be the standard $\sigma$ map. We can suppose that $q_{e}=p_{e}$. Proof. If $q_{e} \neq p_{e}$, consider the new topological space $\sigma^{\prime}$ obtained by shrinking the interval $\left[q_{e}, p_{e}\right]$ to the point $p_{e}$. Note that $\sigma^{\prime}$ is homeomorphic to $\sigma$. We define $g: \sigma^{\prime} \longrightarrow \sigma^{\prime}$ by $g(x)=f(x)$ if $f(x) \in \sigma^{\prime}$, and $g(x)=p_{e}$ if $f(x) \notin$ $\sigma^{\prime}$. By Lemma 6.1, $g$ has a fixed point $x_{0}$ wich verifies the assumptions of Remark 6.2. Then $g$ is a $\sigma$ map such that $P$ is a periodic orbit of period $k$ for $g$ and the endpoint of $\sigma$ belongs to $P$. Since $\operatorname{Per}(g) \subset \operatorname{Per}(f)$, it is sufficient to prove Theorem $1.4(\mathrm{a})$ for the $\sigma$ maps satisfying that $q_{e}=p_{e}$.

Remark 6.4. From now on we can assume that $q_{e}=p_{e}$.

\section{LOOPS AND PERIODS}

In this section we shall study the relation between the periodic points of $f$ and the loops of some $f$-graphs.

As usual if $U \subset \sigma$ we denote by $\operatorname{Int}(U), \mathrm{Cl}(U)$ and by $\partial U$, the interior, the closure and the boundary of $U$ respectively. If $U$ is a finite subset of $\sigma$ we denote by $\operatorname{Card}(U)$ the cardinality of $U$.

We will use the following proposition for the study of $\sigma$ maps with $0 \in$ $P \cup\left\{x_{0}\right\}$.

Proposition 7.1. Let $f$ be the standard $\sigma$ map. Suppose that $0 \in P \cup\left\{x_{0}\right\}$. Let $J_{0} \rightarrow J_{1} \rightarrow \cdots \rightarrow J_{n}=J_{0}$ be a non-repetitive loop of length $n$ in the $f$-graph where the $J_{i}$ 's are basic intervals associated to $P \cup\left\{x_{0}\right\}$. If at least one $J_{i}$ does not contain $x_{0}$, then $f$ has a periodic point of period $n$.

Proof. By Lemma 3.3, there exists $x \in J_{0}$ such that $f^{n}(x)=x$, and $f^{i}(x) \in J_{i}$ for $i \in\{1,2, \cdots, n-1\}$. If $x$ has period $n$, we are done. So suppose that $x$ has period $s$ with $s$ a proper divisor of $n$. Notice that $x \neq x_{0}$, because $x_{0}$ is a fixed point and at least one $J_{i}$ does not contain $x_{0}$. If $x \in \operatorname{Int}\left(J_{0}\right)$, then $\operatorname{Orb}_{f}(x) \cap\left(P \cup\left\{x_{0}\right\}\right)=\varnothing$. Since 0 is not a periodic point having orbit different from $P$, each $f^{i}(x)$ is on a unique basic interval and consequently the loop is repetitive, in contradiction with the hypotheses. Hence, the only possibility is $\operatorname{Orb}_{f}(x)=P$. So $\operatorname{Orb}_{f}(x)=P$ and $k=s>3$. Furthermore, by Remark 6.4 we may assume that $x$ is the endpoint of $\sigma$.

Let $K_{0} \subset J_{0}$ be the subinterval constructed in the proof of Lemma 3.3. Then $f^{i}(x) \in f^{i}\left(K_{0}\right) \subset J_{i}$ for $i \in\{0,1, \cdots, n-1\}$ and $f\left(K_{0}\right)=K_{1} \subset J_{1}$. Since $x=f^{s}(x) \in f^{s}\left(K_{0}\right) \subset J_{s}$ it follows that $J_{s}$ and $J_{0}$ have a common endpoint $x$, which is the endpoint of $\sigma$, so $J_{0}=J_{s}$. Both sets $K_{0}$ and $f^{s}\left(K_{0}\right)$ are contained in $J_{0}$ and contain $x$, an endpoint of $J_{0}$. Therefore $L=K_{0} \cap f^{s}\left(K_{0}\right)$ must be a closed interval. In fact, $L$ is either $K_{0}$ or $f^{s}\left(K_{0}\right)$. Clearly $f^{i}(L) \subset f^{i}\left(K_{0}\right) \subset J_{i}, f^{i}(L) \subset f^{s+i}\left(K_{0}\right) \subset J_{s+i}$ and $f^{i}(L)$ is a closed interval for $i \in\{0,1, \cdots, s-1\}$. Thus $J_{i}=J_{s+i}$ for $i \in\{0,1, \cdots, s-1\}$. Repeating this process we get that the loop $J_{0} \rightarrow J_{1} \rightarrow \cdots \rightarrow J_{n}=J_{0}$ is repetitive, because $s$ divides $n$, in contradiction with the assumptions. 
A sequence of subsets $W_{0}, W_{1}, \cdots, W_{n}=W_{0}$ of $\sigma$ is non-repetitive if there is no integer $i, 0<i<n$, such that $i$ divides $n$ and $W_{j+i}=W_{j}$ for all $j$, $0 \leq j \leq n-i$.

We will using the following result for the $\sigma$ maps with $0 \notin P \cup\left\{x_{0}\right\}$.

Proposition 7.2. Let $f$ be the standard $\sigma$ map. Suppose that $0 \notin P \cup\left\{x_{0}\right\}$. Let $J_{0}, J_{1}, \cdots, J_{n}=J_{0}$ be closed subintervals of $\sigma$, each one contained in a basic interval associated to $P \cup\left\{x_{0}\right\}$ and such that $J_{i} \rightarrow J_{i+1}$ for $0 \leq i \leq n-1$. For each $i, 0 \leq i \leq n$, let $V_{i}$ the component of $\sigma \backslash\left(P \cup\left\{x_{0}\right\}\right)$ containing $\operatorname{Int}\left(J_{i}\right)$ and let $W_{i}=\mathrm{Cl}\left(V_{i}\right)$. Suppose that at least one $W_{i}$ does not contain 0 (i.e. $W_{i}$ is an interval) and at least one $W_{i}$ does not contain $x_{0}$. If the sequence $W_{0}, W_{1}, \cdots, W_{n}=W_{0}$ is non-repetitive, then $f$ has a periodic point of period $n$.

Proof. Without loss of generality we can suppose that $J_{0} \subset W_{0}$ and that $W_{0}$ is an interval (i.e., $W_{0}$ does not contain 0 ). By Lemma 3.3, $f^{n}$ has a fixed point $x$ such that $f^{i}(x) \in K_{i} \subset J_{i}$ for $i \in\{0,1, \cdots, n-1\}$, where the subintervals $K_{i}$ 's are defined in the proof of Lemma 3.3. If $x$ has period $n$, we are done. So suppose that $x$ has period $s, s$ a proper divisor of $n$. Notice that $x \neq x_{0}$ because $x_{0}$ is a fixed point and at least one $W_{i}$ does not contain $x_{0}$. If $x \in \operatorname{Int}\left(J_{0}\right)$, then each $f^{i}(x)$ belongs to a unique $W_{i}$ and therefore the sequence $W_{0}, W_{1}, \cdots, W_{n}=W_{0}$ is repetitive, because $s$ divides $n$. So we must have $\operatorname{Orb}_{f}(x)=P$ and $k=s$.

By Remark 6.4 we can suppose that $x$ is the endpoint of $\sigma$. Then, we note that now $W_{0}$ is not necessarily an interval. Since $x=f^{s}(x) \in J_{s} \subset W_{s}$ and $x \in J_{0} \subset W_{0}, x$ is a common endpoint of $W_{0}$ and $W_{s}$. So $W_{0}=W_{s}$. We have $x \in K_{0} \subset J_{0} \subset W_{0}, f^{s}(x)=x \in K_{s} \subset J_{s} \subset W_{s}=W_{0}$. Note that $K_{0}$ and $f^{s}\left(K_{0}\right)=K_{s}$ are intervals and have $x$ as common endpoint. Therefore $L=K_{0} \cap f^{s}\left(K_{0}\right)$ is an interval, either $K_{0}$ or $f^{s}\left(K_{0}\right)$. Furthermore $f^{i}(L)$ is an interval for $0 \leq i \leq s$. Clearly $f^{i}(L) \subset f^{i}\left(K_{0}\right) \subset J_{i}, f^{i}(L) \subset f^{s+i}\left(K_{0}\right) \subset J_{s+i}$ and so $J_{i} \cap J_{s+1}$ contains an interval. Consequently $W_{i}=W_{s+i}$ for $0 \leq i \leq s$. Repeating this process we get that the sequence $W_{0}, W_{1}, \cdots, W_{n}=W_{0}$ is repetitive, in contradiction with the hypotheses.

\section{REDUCTION TO MONOTONE MAPS}

The graph of a $\sigma$ map $g$ is the subset $\{(x, g(x)): x \in \sigma\}$ of $\sigma \times \sigma$, see Figure 8.1. The segment $p_{e} p_{1}$ represents $I$, and the segment $p_{1} p_{2}$ with the points $p_{1}$ and $p_{2}$ identified to the branching point 0 represents $O$. Roughly speaking we think in the graph of a $\sigma$ map like the graph of an interval map from $\left[p_{e}, p_{2}\right]$ into itself with the above identifications. This allows us to talk about local or absolute maximum or minimum for a $\sigma$ map in the same way as for interval maps. Thus, for instance in the points $p$ and $q$ the $\sigma$ map $g$ represented in Figure 8.1 has a local minimum and maximum with values $m$ and $M$ respectively.

Let $g$ be a $\sigma$ map and let $[a, b]$ a closed subinterval of a basic interval associated to $P \cup\left\{x_{0}\right\} \cup\{0\}$. We will say that $g$ is non-monotone in $[a, b]$ if there exist two different points $c, d \in[a, b]$ such that $g(c)=g(d)$, and $g$ is not constant in the subinterval $[c, d] \subset[a, b]$. Otherwise, we say that $g$ is monotone in $[a, b]$. 


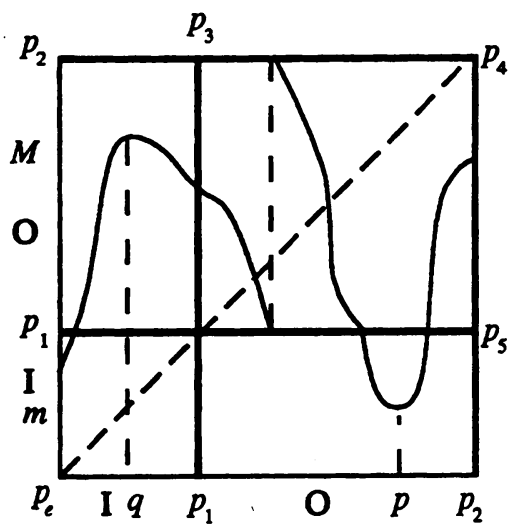

FIGURE 8.1. The graph of a $\sigma$ map.

Proposition 8.1. Let $f$ be the standard $\sigma$ map. Let $U$ be an open subinterval of a basic interval associated to $P \cup\left\{x_{0}\right\} \cup\{0\}$. Suppose that $U=(a, b)$ with $f(a)=f(b)=c, f$ non-monotone in $U$, and $f(d) \neq c$ for $d \in(a, b)$. Let $g$ be the $\sigma$ map defined by $\left.g\right|_{\sigma \backslash U}=\left.f\right|_{\sigma \backslash U}$, and $\left.g\right|_{U}$ is constant and equal to $c$. Then $\operatorname{Per}(g) \subset \operatorname{Per}(f)$.

Proof. Let $x$ be a periodic point of $g$ of period $n>1$. If $c \notin \operatorname{Orb}_{g}(x)$, then $x$ is a periodic point of period $n$ for $f$ and we are done. So, suppose that $c \in \operatorname{Orb}_{g}(x)$; i.e., $c$ is a periodic point of period $n$ for $g$. We shall prove that $n \in \operatorname{Per}(f)$. Set $x_{i}=g^{i}(c)$ for $0 \leq i \leq n$. Therefore $g^{i}(c) \notin U$ for $1 \leq i \leq n-2$ (otherwise $c$ has not period $n$ for $g$ ). Thus $x_{i}=f\left(x_{i-1}\right)=f^{i}(c)$ for $0 \leq i \leq n-1$. If $f\left(x_{n-1}\right)=c$, then $c$ is a periodic point of period $n$ for $f$ and we are done. So, suppose that $f\left(x_{n-1}\right) \neq c$. Then $x_{n-1} \in U$. We claim that $g^{n-1} \circ f$ has a fixed point in $\mathrm{Cl}(U)$. If $\left(g^{n-1} \circ f\right)(\mathrm{Cl}(U)) \subset \mathrm{Cl}(U)$, the claim is clear because $\mathrm{Cl}(U)$ is a closed interval and $g^{n-1} \circ f$ is continuous (see Lemma 3.2). Now we assume that $\left(g^{n-1} \circ f\right)(\mathrm{Cl}(U)) \nsubseteq \mathrm{Cl}(U)$. Also we suppose that there are no fixed points of $\left.\left(g^{n-1} \circ f\right)\right|_{\mathrm{C}(U)}$. So in order to prove the claim we must obtain a contradiction.

Since $\left(g^{n-1} \circ f\right)(a)=\left(g^{n-1} \circ f\right)(b)=x_{n-1}, \quad\left(g^{n-1} \circ f\right)(\mathrm{Cl}(U)) \nsubseteq \mathrm{Cl}(U)$ and $\left.\left(g^{n-1} \circ f\right)\right|_{\mathrm{Cl}(U)}$ has no fixed points, the graph of $\left.\left(g^{n-1} \circ f\right)\right|_{\mathrm{Cl}(U)}$ must be as in Figure 8.2.

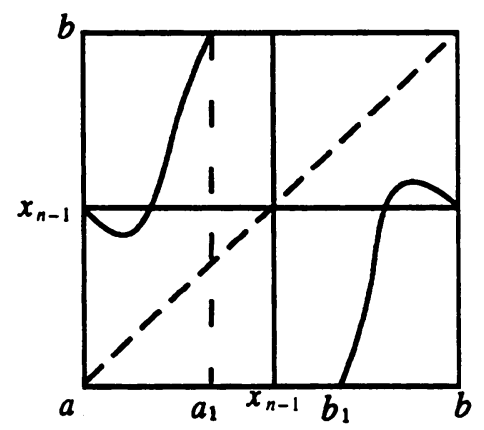

FIGURE 8.2. The graph of $\left.\left(g^{n-1} \circ f\right)\right|_{\mathrm{Cl}(U)}$. 
Now we consider $a_{1} \in \mathrm{Cl}(U)$ such that $\left(g^{n-1} \circ f\right)\left(a_{1}\right)=b$ and $\left[a, a_{1}\right)$ does not contain points $d$ satisfying $\left(g^{n-1} \circ f\right)(d)=b$. Set $J_{1}=\left[a, a_{1}\right]$. We denote by $J_{2}$ the subinterval of $\mathrm{Cl}(U)$ of the form $\left[b_{1}, b\right]$ such that $f\left(J_{1}\right)=f\left(J_{2}\right)$, such interval exists because $\left.f\right|_{U}$ does not contain any neighbourhood of $c$ and $f(\mathrm{Cl}(U)) \nsubseteq \mathrm{Cl}(U)$. Consequently $\left(g^{n-1} \circ f\right)\left(J_{1}\right)=\left(g^{n-1} \circ f\right)\left(J_{2}\right)$. A contradiction with Figure 8.2. Hence the claim is proved.

Let $x$ be the fixed point of $\left.\left(g^{n-1} \circ f\right)\right|_{\mathrm{Cl}(U)}$. We shall prove that $x$ is a periodic point of period $n$ for $f$. We define the sets $Z_{i}=g^{-(n-1-i)}(\mathrm{Cl}(U))$ for $0 \leq i \leq n-1$. Since $g(\mathrm{Cl}(U))=c$, we have $g^{n-i}\left(Z_{i}\right)=g^{n-i-n+1+i}(\mathrm{Cl}(U))=c$ and $g^{n}\left(Z_{i}\right)=x_{i}$. Therefore, the sets $Z_{i}$ must be pairwise disjoint. Since $x \in \mathrm{Cl}(U)$, from $x=\left(g^{n-1} \circ f\right)(x)=g^{(n-1-i)}\left(\left(g^{i} \circ f\right)(x)\right) \in \mathrm{Cl}(U)$ we get $\left(g^{i} \circ f\right)(x) \in Z_{i}$ for $0 \leq i \leq n-1$. Then, the points $\left(g^{i} \circ f\right)(x)$ are all different for $0 \leq i \leq n-1$. Note that $f$ and $g$ coincide in $\sigma \backslash U$, thus $f$ and $g$ coincide in every $Z_{i}$ for $0 \leq i \leq n-2$. Therefore $\left(g^{i} \circ f\right)(x)=f^{i+1}(x) \in Z_{i}$. In particular, for $i=n-2$ we have $\left(g^{n-2} \circ f\right)(x)=f^{n-1}(x) \in Z_{n-2}$ and so $f^{n}(x)=\left(g^{n-1} \circ f\right)(x)=x$; i.e., $n \in \operatorname{Per}(f)$.

Now to finish the proof of the proposition we must show that $g$ is a $\sigma$ map. Since $\left\{0, x_{0}\right\} \nsubseteq U, f(0)=g(0)$ and $f\left(x_{0}\right)=g\left(x_{0}\right)=x_{0}$, so $g$ has fixed points. If $f(0) \in I$ then $g(0) \in I$ and $g$ is a $\sigma$ map. If $f(0) \notin I$ then $x_{0} \in \mathbf{O}$. Hence, again $g$ is a $\sigma$ map.

We consider $\sigma$ as a metric space. We assume for convenience that our metric, called $\delta$ on $\sigma$ satisfies the property that if $z$ is in the interval $[x, y]$, then $\delta(x, y)=\delta(x, z)+\delta(z, y)$. Let $\left(f_{i}\right)_{i \in \mathbb{N}}$ be a sequence of $\sigma$ maps, and let $f$ be the standard $\sigma$ map. The Cauchy's Criterion for uniform convergence says that the sequence $\left(f_{i}\right)_{i \in \mathbb{N}}$ converges uniformly in $\sigma$ if and only if for every $\varepsilon>0$, there exists $n_{0} \in \mathbb{N}$ such that $\delta\left(f_{i}(x), f_{j}(x)\right)<\varepsilon$ for all $x \in \sigma$, whenever $i, j \geq n_{0}$ (see Theorem 7.8 of [R]). Another well-known result is the following: If $\left(f_{i}\right)_{i \in \mathbb{N}}$ converges uniformly to $g$ in $\sigma$, then $g$ is a sigma map (see Theorem 7.12 of $[\mathbf{R}]$ ).

Proposition 8.2. Let $f$ be the standard $\sigma$ map. There exists a $\sigma$ map $g$ such that $\left.g\right|_{P \cup\left\{x_{0}\right\} \cup\{0\}}=\left.f\right|_{P \cup\left\{x_{0}\right\} \cup\{0\}},\left.g\right|_{J}$ is monotone for each basic interval $J$ associated to $P \cup\left\{x_{0}\right\} \cup\{0\}$, and $\operatorname{Per}(g) \subset \operatorname{Per}(f)$.

Proof. We define inductively $\sigma$ maps $f_{i}$ as follows. Set $f_{1}=f$ and suppose that $f_{i}$ has been defined such that $\left.f_{i}\right|_{P \cup\left\{x_{0}\right\} \cup\{0\}}=\left.f\right|_{P \cup\left\{x_{0}\right\} \cup\{0\}}$ and $\operatorname{Per}\left(f_{i}\right) \subset$ $\operatorname{Per}(f)$. If $f_{i}$ is monotone on each basic interval associated to $P \cup\left\{x_{0}\right\} \cup\{0\}$ for some $i$, then we are done. Otherwise, there exist two different points $a, b \in$ Int $(J)$, for some basic interval $J$ such that $f_{i}(a)=f_{i}(b)=c, f_{i}$ is not constant on $[a, b] \subset J$ and $f([a, b])$ does not contain any neighbourhood of $c$. We can suppose that $(a, b)=\left(a_{i}, b_{i}\right)$ is the largest subinterval of $J$ with the above properties. Let $f_{i+1}$ be the result of applying Proposition 8.1 to $U=\left(a_{i}, b_{i}\right)$, so $f_{i+1}$ is constant on $\left[a_{i}, b_{i}\right]$. Note that since the interval $\left[a_{i}, b_{i}\right]$ was select as large as possible, the intervals $\left[a_{i}, b_{i}\right]$ are pairwise disjoint. Furthermore, $f_{i}$ differs from $f_{i+1}$ only on $\left(a_{i}, b_{i}\right)$. Notice that the set of subintervals where $f$ is not monotone is numerable (we can associate a rational number to each one such subintervals). In other words, the set of local maxima and minima of $f$ is numerable. Notice that each $f_{i}$ is a $\sigma$ map from Proposition 8.1. Now we will prove that $\left(f_{i}\right)_{i \in \mathbb{N}}$ converges uniformly to a sigma map $g$. If not, there exists $\varepsilon>0$ such that for every $n \in \mathbb{N}$ if $i, j \geq n$ then $\delta\left(f_{i}, f_{j}\right) \geq \varepsilon$. This means 
that for each $n \in \mathbb{N}$, there exists a subinterval $K$ where $f$ is not monotone and $\max \{\delta(f(x), f(y)): x, y \in K\}>\varepsilon$. So there is an infinite set of pairwise disjoint subintervals $K$ such that $f(K)$ has length larger than $\varepsilon$. There is an accumulation point $p$ of such subintervals, then $f$ is not continuous at $p$, in contradiction with the assumptions. Hence $\left(f_{i}\right)_{i \in \mathbb{N}}$ converges uniformly to a sigma map $g$. Since $f_{i}(0)=f(0)$ and $f_{i}\left(x_{0}\right)=f\left(x_{0}\right)=x_{0}$ for all $i$, it follows that $g(0)=f(0)$ and $g\left(x_{0}\right)=x_{0}$. Hence since $f$ is a $\sigma$ map, also $g$ is a $\sigma$ map.

We shall prove that $\operatorname{Per}(g) \subset \operatorname{Per}(f)$. Let $x$ be a periodic point of $g$ of period $n$. Denote by $D$ the set of points $c_{i} \in \sigma$ with $i \in \mathbb{N}$ for which $f_{i}\left(a_{i}\right)=f_{i}\left(b_{i}\right)=c_{i}, f_{i}$ non-monotone on $\left[a_{i}, b_{i}\right]$ and $\left.f_{i+1}\right|_{\left[a_{i}, b_{i}\right]}=c_{i}$. This means that $D$ is the set where we "cut" $f_{i}$ and obtain the new map $f_{i+1}$. If $c_{i} \notin \operatorname{Orb}_{g}(x)$ for any $c_{i} \in D$, then clearly $x$ is a periodic point of period $n$ for $f_{i}$ for all $i \in \mathbb{N}$ and we are done. So suppose that $c_{i} \in \operatorname{Orb}_{g}(x)$ for some $c_{i} \in D$; i.e., $c_{i}$ is a periodic point of period $n$ for $g$. Then as in the proof of Proposition 8.1 we obtain that $n \in \operatorname{Per}\left(f_{i+1}\right) \subset \operatorname{Per}\left(f_{i}\right) \subset \operatorname{Per}(f)$. This completes the proof.

From now on we will assume that $f$ is monotone on each basic interval associated to $P \cup\left\{x_{0}\right\} \cup\{0\}$. Notice that after this process $x_{0}$ verifies the properties of Section 6. We also remark that if $f$ is monotone on each basic interval $J$ associated to $P \cup\left\{x_{0}\right\} \cup\{0\}$, then $f(J)$ cannot contain $\mathbf{O}$.

\section{REDUCTION TO $\sigma$ MAPS SUCH THAT THERE EXISTS A CLOSED SUBINTERVAL OF $\boldsymbol{\sigma} f$-COVERING $\mathbf{O}$}

Eventually we shall consider the circle as a closed subinterval with the endpoints identified to a point.

Let $f$ be the standard $\sigma$ map. Let $V=\left[v_{1}, v_{2}\right]$ be a subinterval of $\sigma, V$ the largest one such that $f(V) \subset \mathbf{O}, f(V) \neq \mathbf{O}$ and $f(d) \neq 0$ for $d \in\left(v_{1}, v_{2}\right)$. We say that $V$ is an upper subinterval if $f(V) \cap\left(p_{1}, p_{1}+\varepsilon\right)=\varnothing$ for some $\varepsilon>0$ where $p_{1}+\varepsilon \in \mathbf{O}$. We say that $V$ is a down subinterval if $f(V) \cap\left(p_{2}-\varepsilon, p_{2}\right)=\varnothing$ for some $\varepsilon>0$, with $p_{2}-\varepsilon \in \mathbf{O}$.

Let $K$ be a closed subinterval of $\sigma$ such that there exists a closed subinterval $[c, d] \subset K$ with $f([c, d])=\mathbf{0}, f(c)=f(d)=0$, and $f(x) \neq 0$ for all $x \in(c, d)$, then we say that $K f$-covers $\mathbf{O}$, or $K \rightarrow \mathbf{O}$ (or $\mathbf{O} \leftarrow K$ ).

From now on in this section we assume that there are no closed intervals of $\sigma$ $f$-covering $\mathbf{O}$, and under this assumption we prove Theorem 1.4(a).

Since $f$ is monotone on each basic interval associated to $P \cup\left\{x_{0}\right\} \cup\{0\}$, $f$ has finitely many local extrema (see Section 8); and consequently finitely many upper and down subintervals. Let $U_{1}, \ldots, U_{r}$ and $D_{1}, \ldots, D_{s}$ be the upper and down subintervals of $f$ respectively. Let $m_{i}$ be the absolute minimum of $\left.f\right|_{U_{i}}$ and $M_{i}$ the absolute maximum of $\left.f\right|_{D_{i}}$. We denote by $m=\min \left\{m_{1}, \cdots m_{r}\right\}$ and $M=\max \left\{M_{1}, \cdots, M_{s}\right\}$.

Lemma 9.1. With the above notation we have that $m \neq 0$ and $M \neq 0$.

Proof. Suppose $m=0$, then by continuity of $f$, there exists an $i \in\{1, \cdots, r\}$ such that $f\left(U_{i}\right)=\mathbf{0}$, in contradiction with the hypotheses. In the same way, we get that $M \neq 0$.

Now we shall consider an unfolding of $\sigma$ as follows. Define the graph $\tilde{\sigma}=$ $G_{1} \cup G_{2} \cup G_{3}$ where 


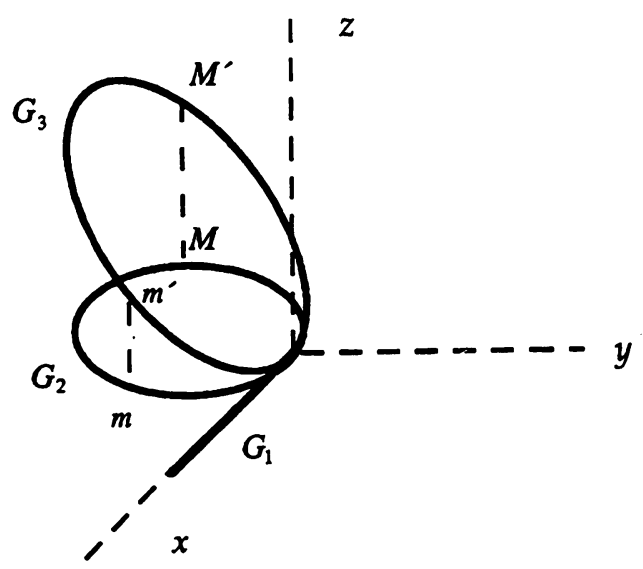

FIGURE 9.1. The unfolding of $\sigma$.

$$
\begin{array}{ll}
G_{1}=\left\{(x, y, z) \in \mathbb{R}^{3}: z=0,\right. & y=0, \quad 0 \leq x \leq 1\}, \\
G_{2}=\left\{(x, y, z) \in \mathbb{R}^{3}: z=0,\right. & \left.x^{2}+(y+1)^{2}=1\right\}, \\
G_{3}=\left\{(x, y, z) \in \mathbb{R}^{3}: y=z,\right. & \left.x^{2}+(y+1)^{2}=1\right\}
\end{array}
$$

see Figure 9.1.

Clearly $G_{1} \cup G_{2}$ is homeomorphic to $\sigma$, so we identify $\sigma$ with $G_{1} \cup G_{2}$. Consider the projection $\pi: \tilde{\sigma} \longrightarrow \sigma$ defined by $\pi(x, y, z)=(x, y, 0)$. We denote by $m^{\prime}$ and $M^{\prime}$ the points of $G_{3}$ such that $\pi\left(m^{\prime}\right)=m$ and $\pi\left(M^{\prime}\right)=$ $M$. For the standard $\sigma$ map $f$ we define $\tilde{f}: \sigma \longrightarrow \tilde{\sigma}$ as follows. Since there are no closed subintervals of $\sigma \quad f$-covering $\mathbf{O}$, and $f$ is monotone in each basic interval associated to $P \cup\left\{x_{0}\right\} \cup\{0\}$, there exists a finite partition of $\sigma$ into closed subintervals $V_{i}$ for $i=1, \cdots, n$ (i.e., $\sigma=\bigcup_{i=1}^{n} V_{i}$ and $\operatorname{Int}\left(V_{i}\right) \cap \operatorname{Int}\left(V_{j}\right)=\varnothing$ if $\left.i \neq j\right)$ such that

(i) either $f\left(V_{i}\right) \subset \mathbf{I}$,

(ii) or $f\left(V_{i}\right) \subset \mathbf{O}$, and $f(d) \neq 0$ for $d \in \operatorname{Int}\left(V_{i}\right)$,

(iii) the subintervals $V_{i}$ are the largest ones satisfying (i) or (ii).

If $V_{i}$ is one of the subintervals of $\sigma$ safisfying (i), then define $\left.\tilde{f}\right|_{V_{i}}=\left.f\right|_{V_{i}}$. Let $V_{i}$ be a subinterval of $\sigma$ satisfying (ii). Then $V_{i}$ is either an upper subinterval or a down subinterval. Suppose that $V_{i}$ is an upper subinterval. Then for every $r \in V_{i}$ we define $\tilde{f}(r)=q^{\prime} \in G_{3}$ such that $\pi\left(q^{\prime}\right)=f(r) \in G_{2}$. Now assume that $V_{i}$ is a down subinterval. Then for every $r \in V_{i}$, we define $\tilde{f}(r)=f(r) \in G_{2}$. Clearly $\tilde{f}$ is well-defined. Notice that $f=\pi \circ \tilde{f}: \sigma \longrightarrow \sigma$. Define $F=\tilde{f} \circ \pi$ : $\tilde{\boldsymbol{\sigma}} \longrightarrow \tilde{\boldsymbol{\sigma}}$.

Lemma 9.2. The map $F: \tilde{\sigma} \longrightarrow \tilde{\sigma}$ satisfies that $\operatorname{Per}(F)=S\left(n_{s}\right) \cup G\left(n_{g}\right) \cup R\left(n_{r}\right)$ for some $n_{s} \in \mathbb{N}_{s}, n_{g} \in \mathbb{N}_{g}$ and $n_{r} \in \mathbb{N}_{r}$.

Proof. By Lemma 9.1, $0 \notin\{m, M\}$. Furthermore, since there are no closed intervals of $\sigma f$-covering $\mathbf{O}$, we have that $F(\tilde{\boldsymbol{\sigma}})$ is homeomorphic to $\mathbf{I}$ or $\mathbf{Y}$. So, from the Interval Theorem or the $\mathbf{Y}$ Theorem, we can write $\operatorname{Per}(F)=$ $S\left(n_{s}\right) \cup G\left(n_{g}\right) \cup R\left(n_{r}\right)$ for some $n_{s} \in \mathbb{N}_{s}, n_{g} \in \mathbb{N}_{g}$ and $n_{r} \in \mathbb{N}_{r}$.

Now we shall relate the periodic orbits of $f$ with the periodic orbits of $F$.

Lemma 9.3. The map $F: \tilde{\sigma} \longrightarrow \tilde{\sigma}$ satisfies that $k \in \operatorname{Per}(F)$. 
Proof. Let $x \in P$. Then $x$ is a periodic point of period $k$ for $f$. Also we can assume, by Section 5, that $x \in \mathrm{I}($ so $\pi(x)=x)$. We have $f^{k}(x)=x$ and $f^{i}(x) \neq x$ for $1 \leq i<k$. This means that $(\pi \circ \tilde{f})^{k}(\pi(x))=x$. So $\pi \circ(\tilde{f} \circ \pi)^{k}(x)=x$, and by definition $\pi\left(F^{k}(x)\right)=\pi(x)$. Thus $F^{k}(x)=x$. Moreover $F^{i}(x) \neq x$ for $1 \leq i<k$, otherwise $(\tilde{f} \circ \pi)^{i}(x)=x$ for some $i<k$, and so $(\pi \circ \tilde{f})^{i} \circ \pi(x)=\pi(x)$. Hence $f^{i}(\pi(x))=\pi(x)$ for some $1 \leq i<k$ in contradiction with the hypotheses. Therefore $x$ is a periodic point of period $k$ for $F$.

Lemma 9.4. If $x \in \tilde{\sigma}$ is a periodic point of period $n$ for $F$, then $\pi(x) \in \sigma$ is periodic of period $n$ for $f$.

Proof. Set $\operatorname{Orb}_{F}(x)=\left\{p_{1}, p_{2}, \cdots, p_{n}\right\}$ such that $F\left(p_{i}\right)=p_{i+1}$ for $1 \leq$ $i \leq n-1$ and $F\left(p_{n}\right)=p_{1}$. Let $q_{i}=\pi\left(p_{i}\right)$ for $1 \leq i \leq n$. Notice that $\pi\left(p_{i}\right) \neq \pi\left(p_{j}\right)$ if $i \neq j$, otherwise $\pi\left(p_{i}\right)=\pi\left(p_{j}\right)$ and consequently $F\left(p_{i}\right)=$ $(\tilde{f} \circ \pi)\left(p_{i}\right)=(\tilde{f} \circ \pi)\left(p_{j}\right)=F\left(p_{j}\right)$ in contradiction with the fact that $\left\{p_{1}, \cdots, p_{n}\right\}$ is a periodic orbit of period $n$. Then the $q_{i}$ 's are pairwise different and $f\left(q_{i}\right)=(\pi \circ \tilde{f})\left(\pi\left(p_{i}\right)\right)=\pi\left(F\left(p_{i}\right)\right)=\pi\left(p_{i+1}\right)=q_{i+1}$ for $1 \leq i \leq n-1$ and $f\left(q_{n}\right)=q_{1}$. So $\left\{q_{1}, \cdots, q_{n}\right\}=\operatorname{Orb}_{f}(\pi(x))$ is a periodic orbit of period $n$ for $f$.

Proposition 9.5. Let $f$ be the standard $\sigma$ map. Then $S(k), G(k)$ or $R(k)$ is contained in $\operatorname{Per}(f)$.

Proof. By Lemma 9.3, $k \in \operatorname{Per}(F)$; and by Lemma 9.4, $\operatorname{Per}(F) \subset \operatorname{Per}(f)$. Then from Lemma 9.2 $S(k), G(k)$ or $R(k)$ is contained in $\operatorname{Per}(f)$.

Remark 9.6. From now on we can suppose that there exists a closed subinterval $K$ of $\sigma$ such that $K f$-covers $\mathbf{O}$. The interval $K$ is not necessarily basic. Eventually $K$ is the circle.

We remark that the idea of the unfolding of $\sigma$ when there is no closed interval $f$-covering $\mathbf{O}$ comes from the Ph.D. thesis of Paraños [P]. In fact the unfolding of $\sigma$ is essentially equivalent to work with the lifting of $f$. Thus the ideas of Section 3.5 of [ALM2] are closed to the ones developed here.

\section{REDUCTION TO $\sigma$ MAPS SUCH THAT EVERY BASIC INTERVAL OF $T$ IS $f$-COVERED BY SOME BASIC INTERVAL OF $T$}

From now on we denote by $S$ or $S^{\prime}$ the set of all basic intervals associated to $P \cup\left\{x_{0}\right\}$ or $P \cup\left\{x_{0}\right\} \cup\{0\}$ respectively.

We shall work with the basic intervals $A, B, C, L_{1}, L_{2}$ and $L_{3}$ associated to $P \cup\left\{x_{0}\right\} \cup\{0\}$ or $P \cup\left\{x_{0}\right\}$ defined in Section 2 .

In this section we can restrict our attention to the $\sigma$ maps satisfying that each basic interval of $S$ is $f$-covered by some basic interval of $S$; or each basic interval of $S \backslash\left\{L_{j}\right\}$ is $f$-covered by some basic interval of $S \backslash\left\{L_{j}\right\}$ for some $j \in\{1,2,3\}$.

Proposition 10.1. Let $f$ be the standard $\sigma$ map. Suppose that $f(0) \in \operatorname{Int}(D)$ for some $D \in S^{\prime}$. If $D$ is not $f$-covered by any basic interval of $\left\{L_{1} ; L_{2}, L_{3}\right\}$, then there exists a $\sigma$ map $g$ such that $\left.g\right|_{P \cup\left\{x_{0}\right\}}=\left.f\right|_{P \cup\left\{x_{0}\right\}}, g(0) \in \partial D, D$ 
is not $f$-covered by any basic interval of $\left\{L_{1}, L_{2}, L_{3}\right\}, g$ is monotone in each basic interval of $S^{\prime}$ and $\operatorname{Per}(g) \subset \operatorname{Per}(f)$.

Proof. Since $f(0) \in \operatorname{Int}(D)$ and there is no basic interval of $\left\{L_{1}, L_{2}, L_{3}\right\}$ $f$-covering $D$, it follows that there are closed subintervals $A^{\prime} \subset A, B^{\prime} \subset B$ and $C^{\prime} \subset C$ such that $f\left(A^{\prime} \cup B^{\prime} \cup C^{\prime}\right) \subset D$ and the image of the endpoints of $A^{\prime}, B^{\prime}$ and $C^{\prime}$ is $\{f(0), z\}$, where $z$ is an endpoint of $D$. Notice that $A^{\prime} \cup B^{\prime} \cup C^{\prime}$ is homeomorphic to $\mathbf{Y}$. We define $\left.g\right|_{\sigma \backslash\left(A^{\prime} \cup B^{\prime} \cup C^{\prime}\right)}=\left.f\right|_{\sigma \backslash\left(A^{\prime} \cup B^{\prime} \cup C^{\prime}\right)}$ and $\left.g\right|_{A^{\prime} \cup B^{\prime} \cup C^{\prime}}=z$ (in particular $g(0)=z \in \partial D$ ). Clearly $g$ is a $\sigma$ map because $g(0) \in D$ and $g\left(x_{0}\right)=x_{0}$. Since $\left.g\right|_{P}=\left.f\right|_{P}, k \in \operatorname{Per}(g)$. Furthermore $g$ is monotone on each basic interval of $S^{\prime}$ and $\operatorname{Per}(g) \subset \operatorname{Per}(f)$.

Remark 10.2. From now on, by Proposition 10.1, we can assume that there are no basic intervals $D \in S^{\prime}$ satisfying the following properties : $f(0) \in \operatorname{Int}(D)$ and $D$ is not $f$-covered by any basic interval of $\left\{L_{1}, L_{2}, L_{3}\right\}$.

Lemma 10.3. Let $f$ be the standard $\sigma$ map. Then each basic interval $J$ of $S$ (respectively $S^{\prime}$ ) contained in I is $f$-covered by some basic interval of $S$ (respectively $S^{\prime}$ ).

Proof. Since $f(\boldsymbol{\sigma})$ is a connected set, the endpoint of $\mathbf{I}$ belongs to $P$ (see Remark 6.4) and the endpoints of all basic intervals belong to $P \cup\left\{x_{0}\right\}$ (respectively $P \cup\left\{x_{0}\right\} \cup\{0\}$ ), we obtain that every basic interval contained in $\mathbf{I}$ is $f$-covered by some basic interval of $S$ (respectively $S^{\prime}$ ).

Lemma 10.4. Let $f$ be the standard $\sigma$ map. Then each basic interval of $S^{\prime}$ is $f$-covered by some basic interval of $S$.

Proof. Notice that if $0 \in P \cup\left\{x_{0}\right\}$, then $S=S^{\prime}$. We have that each basic interval of $S^{\prime}$ is a subinterval of some basic interval of $S$. So by Lemma 10.3 each basic interval of $S^{\prime}$ contained in $\mathbf{I}$ is $f$-covered by some basic interval of $S$. On the other hand, since there exists a closed subinterval $K$ of $\sigma$ (nonnecessarily basic) such that $K \quad f$-covers $\mathbf{O}$ we have that each basic interval of $S^{\prime} \cap S$ contained in $\mathbf{O}$ is $f$-covered by some basic interval (subinterval of $K$ ) of $S$, this would be shown as in the proof of Lemma 3.1.

Remark 10.5. From now on, by Lemma 10.3 and Lemma 10.4 we can assume that each basic interval of $S^{\prime}$ is $f$-covered by some basic interval of $S$ and $f(\boldsymbol{\sigma})=\boldsymbol{\sigma}$.

Lemma 10.6. Let $f$ be the standard $\sigma$ map. Suppose that $0 \notin P \cup\left\{x_{0}\right\}$. Then at least two intervals of $\left\{L_{1}, L_{2}, L_{3}\right\}$ are $f$-covered by some basic intervals of $S$.

Proof. From Lemma 10.4 each interval $A, B$ and $C$ is $f$-covered by some basic interval of $S$. For each pair $i, j \in\{1,2,3\}, i \neq j, L_{i} \cap L_{j}$ is one interval of $\{A, B, C\}$. We claim that $L_{i}$ or $L_{j}$ is $f$-covered by some basic interval of $S$. Suppose that $L_{i} \cap L_{j}=A$ (the proof is analogous in the other cases). By Remark 10.5 there exists $J \in S$ such that $J \rightarrow A$. First suppose that $J \in S^{\prime}$. Since $f$ is monotone on $J$ and the endpoints of $J$ are not mapped in $\{0\}$, we have that $J \rightarrow B$ or $J \rightarrow C$. Hence $J \rightarrow L_{i}$ or $J \rightarrow L_{j}$.

Finally we can assume that $J \notin S^{\prime}$. If $f(0) \in \operatorname{Int}(A \cup B \cup C)$, then $f(0) \in$ $\operatorname{Int}(D)$ for some $D \in\{A, B, C\}$. By Remark $10.2, D$ is $f$-covered by some interval of $\left\{L_{1}, L_{2}, L_{3}\right\}$. Hence there is $M \in\left\{L_{1}, L_{2}, L_{3}\right\}$ such that $M \rightarrow A$ and either $M \rightarrow B$ or $M \rightarrow C$. So $M \rightarrow L_{i}$ or $M \rightarrow L_{j}$. Otherwise, $f(0) \notin \operatorname{Int}(A \cup B \cup C)$. Therefore there exists $N \in\{A, B, C\}, N \subset J$ such 
that $N \rightarrow A$. By the above arguments $N \rightarrow L_{i}$ or $N \rightarrow L_{j}$. So the claim is proved. Consequently at least two intervals of $\left\{L_{1}, L_{2}, L_{3}\right\}$ are $f$-covered by some basic intervals of $S$.

Lemma 10.7. Let $f$ be the standard $\sigma$ map. Suppose that $0 \notin P \cup\left\{x_{0}\right\}$. Let $J$ be a basic interval of $S$. If there is $L_{j} \in S$ for some $j=1,2,3$ such that $L_{j} \rightarrow J$, then there exists $L_{i}$ for some $i=1,2,3, i \neq j$ such that $L_{i} \rightarrow J$.

Proof. Suppose that $L_{j} \rightarrow J$ for some $j=1,2,3$. If $f(0) \notin \operatorname{Int}(J)$, then $A, B$ or $C f$-covers $J$. Notice that each interval $A, B$ and $C$ belongs to two intervals of $\left\{L_{1}, L_{2}, L_{3}\right\}$. Hence there is $L_{i}, i=1,2,3, i \neq j$, such that $L_{i} \rightarrow J$. So we can assume that $f(0) \in \operatorname{Int}(J)$. Denote by $J_{1}$ and $J_{2}$ the closures of the two connected components of $J \backslash\{f(0)\}$. Set $L_{j}=M_{1} \cup M_{2}$ where $M_{1}, M_{2} \in\{A, B, C\}$, and $M_{1} \rightarrow J_{1}, M_{2} \rightarrow J_{2}$. Then by continuity, the interval $M_{3} \in\{A, B, C\} \backslash\left\{M_{1}, M_{2}\right\} \quad f$-covers either $J_{1}$ or $J_{2}$. So, either $M_{1} \cup M_{3}$ or $M_{2} \cup M_{3} f$-covers $J$, and the result follows.

Proposition 10.8. Let $f$ be the standard $\sigma$ map. Suppose $0 \notin P \cup\left\{x_{0}\right\}$. Then at least one of the following two statements hold.

(a) Each basic interval of $S$ is $f$-covered by some basic interval of $S$.

(b) Each basic interval of $S \backslash\left\{L_{j}\right\}$ for some $j \in\{1,2,3\}$ is $f$-covered by some basic interval of $S \backslash\left\{L_{j}\right\}$.

Proof. If statement (a) does not hold, by Lemma 10.4 there is $L_{j} \in S$ for some $j=1,2,3$ such that there are no basic intervals of $S f$-covering $L_{j}$, and by Lemma 10.6 the above interval $L_{j}$ is unique. If $L_{j} \rightarrow J$ for some $J \in S \backslash\left\{L_{j}\right\}$, then from Lemma 10.7 there exists $L_{i} \in S$ such that $L_{i} \rightarrow J$ and statement (b) holds.

Notice that if $0 \in P \cup\left\{x_{0}\right\}$, by Lemma 10.4 each basic interval of $S=S^{\prime}$ is $f$-covered by some basic interval of $S=S^{\prime}$. So from now on, if $0 \in P \cup\left\{x_{0}\right\}$ we denote by $T$ the collection of all basic intervals of $S=S^{\prime}$. If $0 \notin P \cup\left\{x_{0}\right\}$ we denote by $T$ the collection of all basic intervals of $S$ if (a) of Proposition 10.8 is satisfied, otherwise $T=S \backslash\left\{L_{j}\right\}$ for some $j \in\{1,2,3\}$ such that (b) of Proposition 10.8 is satisfied.

\section{SOME BASIC RESULTS}

In this section we obtain some results wich we are using in the next sections.

Lemma 11.1. Let $f$ be the standard $\sigma$ map. Let $J_{0}$ be a closed subinterval of I with endpoints elements of $P \cup\left\{x_{0}\right\}$. Notice that $J_{0}$ is not necessarily basic. Then there exists a repetitive or non-repetitive loop of length $k$ containing $J_{0}$ formed by closed subintervals of $\boldsymbol{\sigma}$, in general no basic intervals.

Proof. Set $J_{0}=[x, y]$. For each $0<i \leq k$, we define $J_{i}$ recursively as the closed subinterval with endpoints $f^{i}(x)$ and $f^{i}(y)$ such that $J_{i-1} \rightarrow J_{i}$. (Note that if $f^{i}(x)$ or $f^{i}(y)$ belongs to $\mathbf{O}$, then $J_{i}$ perhaps is not unique.) Since $J_{0} \subset \mathrm{I}$, we get $J_{0}=J_{k}$. Thus, we obtain the loop $J_{0} \rightarrow J_{1} \rightarrow \cdots \rightarrow J_{k}=J_{0}$. Of course, in general $J_{i}$ is not a basic interval and the above loop can be repetitive or non-repetitive.

Remark 11.2. Suppose that $g$ is an interval map. Note that if $g^{2}$ has a periodic point $z$ of period $s$, then $z$ is a periodic point for $g$ with period $2 s$ if $s$ is even, and period $s$ or $2 s$ if $s$ is odd. 
Lemma 11.3. Let $f$ be the standard $\sigma$ map. Let $z \in \mathbf{I}$ be a fixed point of $f$. Denote by $D_{1}$ and $D_{2}$ the closures of the two connected components of $\sigma \backslash\{z\}$, such that $D_{1}$ is homeomorphic to I. If $f\left(D_{1}\right)=D_{2}$ and $f\left(D_{2}\right)=D_{1}$, then $S(k) \subset \operatorname{Per}(f)$.

Proof. Notice that the map $\left.f^{2}\right|_{D_{1}}: D_{1} \longrightarrow D_{1}$ is an interval map. Therefore $f$ has no periodic points of odd period except the period 1. So $k$ is even and by Remark 11.2 the points of $P \cap D_{1}$ have period $k / 2$ for $\left.f^{2}\right|_{D_{1}}$. From the Interval Theorem it follows that $S(k / 2) \subset \operatorname{Per}\left(f^{2}\right)$. Since all the periods of $f$ are even except $1, S(k) \subset \operatorname{Per}(f)$.

Lemma 11.4. Let $f$ be the standard $\sigma$ map. Suppose that each basic interval of $T$ is $f$-covered by some basic interval of $T$ different from itself. Suppose that there is $J \in T$ such that $J \rightarrow J$. Then there is a non-repetitive loop of length $n$ for each $n \geq k+1$ containing $J$. Moreover, if $N \in T$ and there is a path starting at $N$ and ending at $J$, then we can assume that the loop of length $n$ contains such a path.

Proof. From Remark 10.5 we have $f(\sigma)=\sigma$. Set $K_{i}=f^{i}\left(J_{0}\right)$ for $i \geq 0$, where $J_{0}=J$. Notice that each $K_{i}$ is a connected set and $\operatorname{Card}\left(K_{1} \cap P\right) \geq 2$. So from the fact that $P$ is a periodic orbit and Remark 10.5, it follows that there exists an integer $r$ such that $K_{0} \subsetneq K_{1} \subsetneq \cdots \subsetneq K_{r}=\sigma$ and $\operatorname{Card}\left(K_{i} \cap P\right) \geq i+1$ for $i<r$. Since $P$ has period $k$ we have that $r \leq \operatorname{Card}\left(K_{r-1} \cap P\right) \leq k$. Since each basic interval of $T$ is $f$-covered by some basic interval different from itself, for each basic interval $J_{i} \in T, J_{i} \subset K_{i} \backslash K_{i-1}$ there exists $J_{i-1} \in T$, $J_{i-1} \subset K_{i-1} \backslash K_{i-2}$ such that $J_{i-1} \rightarrow J_{i}$. By hypotheses there exists $M \in T$, $M \neq J_{0}$ such that $M \rightarrow J_{0}$. Hence there is a loop of length $l \leq r+1 \leq k+1$ containing $J_{0}$. By construction, this loop is formed by pairwise different basic intervals and so is non-repetitive. The above loop of length $l$ together with the loop $J_{0} \rightarrow J_{0}$ give us a non-repetitive loop of length $n$ for each $n \geq$ $k+1$ containing $J_{0}$. The last statement of the lemma follows easily by the construction of the non-repetitive loop of length $n$.

Lemma 11.5. Let $f$ be the standard $\sigma$ map. Suppose that each basic interval of $T$ is $f$-covered by some basic interval of $T$ different from itself. Suppose that there are $I_{1}, I_{2} \in T$ such that $I_{1} \cap I_{2}=\left\{x_{0}\right\}$ and $I_{1} \rightleftarrows I_{2}$. If $J \in T$, then there is a path of length $l \leq k-1$ starting at one of the intervals $I_{1}, I_{2}$ and ending at $J$.

Proof. By Remark 10.5, $f(\sigma)=\sigma$. Set $K_{i}=f^{i}\left(I_{1} \cup I_{2}\right)$ for $i \geq 0$. Notice that $K_{i}$ is connected for all $i \in \mathbb{N}$ and $\operatorname{Card}\left(K_{1} \cap P\right) \geq 3$. Since $P$ is a periodic orbit, from Remark 10.5 it follows that there exists an integer $r$ such that $K_{0} \subsetneq K_{1} \subsetneq \cdots \subsetneq K_{r}=\sigma$ and $\operatorname{Card}\left(K_{i} \cap P\right) \geq i+2$ for $i<r$. From the fact that $P$ has period $k$ we get that $r+1 \leq \operatorname{Card}\left(K_{r-1} \cap P\right) \leq k$, and so $r \leq k-1$. From the assumptions, for each basic interval $J_{i} \in T, J_{i} \subset K_{i} \backslash K_{i-1}$ there exists $J_{i-1} \in T, J_{i-1} \subset K_{i-1} \backslash K_{i-2}$ such that $J_{i-1} \rightarrow J_{i}$. Hence given $J \in T$ there is a path of length $l \leq r \leq k-1$ starting at one of the intervals $I_{1}$ or $I_{2}$ and ending at $J$.

We denote by $2 \mathbb{N}$ the set of all even natural numbers.

Lemma 11.6. Let $f$ be the standard $\sigma$ map. Let $K \subset \mathrm{I}$ and $M, N \subset \mathrm{O}$ be closed subintervals such that $M \rightleftarrows K \rightleftarrows N$. Suppose that $K$ has endpoints elements of $P \cup\left\{x_{0}\right\}$ and $K$ does not contain any proper closed subinterval with 
endpoints in $P \cup\left\{x_{0}\right\} \quad f$-covering $M$ and $N$. Assume that $M$ and $N$ have disjoint interiors and do not contain any proper closed subinterval $f$-covering $K$. Then Theorem 1.4(a) holds.

Proof: First suppose that at least one interval of $\{M, N, K\}$ does not contain $x_{0}$. Then the subgraph $M \rightleftarrows K \rightleftarrows N$ give us a non-repetitive loop $J_{0}=K \rightarrow$ $J_{1} \rightarrow \cdots \rightarrow J_{n}=K$ of length $n$ for each $n$ even such that $J_{i}=K$ if $i$ is even; $J_{1}=M$ and $J_{i}=N$ if $i>1$ is odd and $x_{0} \in N$; and $J_{1}=N$ and $J_{i}=M$ if $i>1$ is odd and $x_{0} \in M$. By Lemma 3.3 there exists $x \in K$ such that $f^{n}(x)=x$ and $f^{i}(x) \in J_{i}$ for $1 \leq i \leq n-1$. Since $x_{0}$ does not belong to some interval of $\{M, N, K\}$, and the loop is non-repetitive, $x$ has period $n$. So $2 \mathbb{N} \subset \operatorname{Per}(f)$. If $k$ is even we are done, because $S(k) \subset \operatorname{Per}(f)$. So suppose $k$ odd. Since $K \subset I$ and $K$ has endpoints elements of $P \cup\left\{x_{0}\right\}$, by Lemma 11.1 there exists a repetitive or non-repetitive loop of length $k$ containing $K$. This loop together with the loops $M \rightleftarrows K \rightleftarrows N$ give us a non-repetitive loop of length $n$ for each $n>k$ odd. Such a loop can be chosen in such a way that at least one of its intervals (non necessarily basic intervals) does not contain $x_{0}$. Hence we get that $S(k) \subset \operatorname{Per}(f)$.

Finally suppose that $x_{0} \in M \cap N \cap K$. In particular, this means that $x_{0}=0$. So we get that $A \subset K, B \rightarrow A \leftarrow C$ and either $A \rightarrow B$ or $A \rightarrow C$. Without loss of generality we can assume that $B \rightleftarrows A \leftarrow C$. Also we can suppose that there exists $z \in \mathbf{I}$ such that $f(z) \in \mathbf{I}$ or $z \in \mathbf{O}$ such that $f(z) \in \mathbf{O}$; otherwise $f(\mathbf{I})=\mathbf{O}$ and $f(\mathbf{O})=\mathbf{I}$ and, by Lemma 11.3, $S(k) \subset \operatorname{Per}(f)$. Therefore since $f$ is monotone on each basic interval of $S^{\prime}$, there exists $J \in S$ such that $0 \notin J$ and either $J \rightarrow A \cup B$ or $J \rightarrow A \cup C$. From Remark 10.5 and Lemma 11.5, there is a path of length $l \leq k-1$ starting in one of the intervals $A$ or $B$ and ending in $J$. Then we consider the non-repetitive loops $A \rightleftarrows B$, $A \rightarrow \cdots \rightarrow J \rightarrow A$ and either $B \rightarrow \cdots \rightarrow J \rightarrow B$ or $C \rightarrow \cdots \rightarrow J \rightarrow C$ of lengths either $2, l+1 \leq k, l+2 \leq k+1$ or $2, l+2 \leq k+1, l+1 \leq k$, be the rest of this section we will assume that $0 \notin P \cup\left\{x_{0}\right\}$ respectively. By Proposition 7.1 we obtain $B(k) \subset \operatorname{Per}(f)$.

In the rest of the section we will assume that $0 \notin P \cup\left\{x_{0}\right\}$.

Lemma 11.7. Let $f$ be the standard $\sigma$ map. Let $M \in S^{\prime}$ be such that $f(0) \notin$ $\operatorname{Int}(M)$. If $\operatorname{Int}(M)$ contains a periodic point of period $n>1$, then there exists $L \in S^{\prime}, L \neq M$ such that $L \rightarrow M$.

Proof. From the hypotheses and since $f$ is monotone in $M$ we can choose a periodic point $z$ of period $n$ such that $z \in \operatorname{Int}(M)$ and $f^{n-1}(z) \in L$ for some $L \in S^{\prime}, L \neq M$. Since $z$ has period $n$ and $f$ is monotone, we have $L \rightarrow M$.

Proposition 11.8. Let $f$ be the standard $\sigma$ map. Suppose that there exists $J \in S$ such that $J \rightarrow J$ and $J$ is not $f$-covered by any basic interval of $T$ different from itself.

(a) If $J \notin\left\{L_{1}, L_{2}, L_{3}\right\}$ then Theorem 1.4(a) holds.

(b) If $J \in\left\{L_{1}, L_{2}, L_{3}\right\}$ then we assume that $f(0) \notin \operatorname{Int}(J)$. Therefore Theorem 1.4(a) holds.

Proof. Suppose that $J \notin\left\{L_{1}, L_{2}, L_{3}\right\}$. Then $J \in S^{\prime}$. Since $J$ is not $f$ covered by any $M \in T \backslash\{J\}$, from Lemma $10.7, J$ is not $f$-covered by any $M \in S \backslash\{J\}$, and consequently $J$ is not $f$-covered by any $M \in S^{\prime} \backslash\{J\}$. Therefore, from Remark 10.2 we get that $f(0) \notin \operatorname{Int}(J)$. 
We separate the proof into the following three cases.

Case 1: $J \subset \mathbf{O}$ and $J \neq L_{3}$. Then $J \in S^{\prime}$. Since $f(0) \notin \operatorname{Int}(J)$, by Lemma 11.7, Int $(J)$ does not contain periodic points of period $n>1$. Since $f$ is monotone on each basic interval of $S^{\prime}, J \rightarrow J, J$ is not $f$ covered by any basic interval of $S^{\prime}$ different from itself and $f(0) \notin \operatorname{Int}(J)$, we have that $f(\sigma \backslash \operatorname{Int}(J)) \cap \operatorname{Int}(J)=\varnothing$. Then we consider the $Y$ map $g=$ $\left.f\right|_{\sigma \backslash \operatorname{Int}(J)}: \sigma \backslash \operatorname{Int}(J) \longrightarrow \sigma \backslash \operatorname{Int}(J)$. Clearly $g$ is well-defined, $k \in \operatorname{Per}(g)$ and $\operatorname{Per}(g)=\operatorname{Per}(f)$. By the $\mathbf{Y}$ Theorem the result follows.

Case 2: $J \subset I$. Then $J \in S^{\prime}$. Since $J \rightarrow J$ there exists a fixed point $z \in J$ of $f$. Denote by $D_{1}$ and $D_{2}$ the closures of the two connected components of $\boldsymbol{\sigma} \backslash\{z\}$ such that $D_{1}$ is homeomorphic to $I$. Moreover since $P$ is a periodic orbit it is not possible that $f\left(D_{1}\right) \subset D_{1}$. So, since $J \rightarrow J, J$ is not $f$-covered by any $M \in S^{\prime} \backslash\{J\}, f(0) \notin \operatorname{Int}(J)$ and from Remark 10.2 we have that $f\left(D_{1}\right)=D_{2}$ and $f\left(D_{2}\right)=D_{1}$. Hence, by Lemma 11.3 , statement (a) follows.

Case 3: $J \in\left\{L_{1}, L_{2}, L_{3}\right\}$. Then $f(0) \notin \operatorname{Int}(J)$. Therefore, since $J \rightarrow J$ we have that $A, B$ or $C f$-covers $J$. In particular, $J$ is $f$-covered by a basic interval $L \in\left\{L_{1}, L_{2}, L_{3}\right\}$ different from itself. So $L \notin T$. Set $J=$ $M_{1} \cup M_{2}, L=M_{1} \cup M_{3}$ where $M_{1} \rightarrow M_{1} \rightarrow M_{2}$ and $\left\{M_{1}, M_{2}, M_{3}\right\}=$ $\{A, B, C\}$. Therefore $M_{2} \cup M_{3} \in T$. We claim that $M_{2}$ does not $f$-cover $M_{1}$. Otherwise $M_{2} \rightarrow M_{1}$, and since $f(0) \notin \operatorname{Int}(J) \quad M_{2}$ also $f$-covers $M_{2}$ or $M_{3}$ and consequently $M_{2} \cup M_{3} f$-covers $J$ or $L$. If $M_{2} \cup M_{3} \rightarrow J$ we have a contradiction with the fact that $M_{2} \cup M_{3} \in T$ and $J$ is not $f$-covered by any $M \in T \backslash\{J\}$. Suppose that $M_{2} \cup M_{3} \rightarrow L$. Since $L \notin T$, from the definition of $T$ we get that $T=S \backslash\{L\}$ and that $L$ is not $f$-covered by any basic interval of $S$, in contradiction with $M_{2} \cup M_{3} \in T$ and $M_{2} \cup M_{3} \in L$. So the claim is proved.

Since the only basic intervals $f$-covering $J$ are $J$ and $L \notin T$, there are no basic intervals of $S^{\prime} \quad f$-covering $M_{1} \in S^{\prime}$ different from itself. So the proof of (b) follows in the same way as the proof of Cases 1 and 2.

Remark 11.9. In what follows, from Proposition 11.8, we can assume that if $J$ is a basic interval of $T$ such that either $J \subset \mathbf{O}$ and $J \neq L_{3}$, or $J \subset \mathbf{I}$, or $J \in\left\{L_{1}, L_{2}, L_{3}\right\}$ and $f(0) \notin \operatorname{Int}(J)$, then $J$ is $f$-covered by some basic interval of $T$ different from itself.

Proposition 11.10. Let $f$ be the standard $\sigma$ map. Suppose that each basic interval of $T$ is $f$-covered by some basic interval of $T$ different from itself. If there exists a basic interval $J \in S \cap S^{\prime} \quad f$-covering itself, then Theorem 1.4(a) holds.

Proof. By Lemma 11.4 there exists a non-repetitive loop of length $n$ for each $n \geq k+1$ containing $J$. Since $J \in S \cap S^{\prime}, 0 \notin J$. Moreover, if $x_{0} \in J$, by the monotonicity of $f$ on each interval of $S^{\prime}, J$ does not $f$-cover any basic interval of $T$ containing $x_{0}$ different from $J$. Then the loop verifies the hypotheses of Proposition 7.2 and the result follows.

Corollary 11.11. Let $f$ be the standard $\sigma$ map. Suppose that each basic interval of $T$ is $f$-covered by some basic interval of $T$ different from itself and that $x_{0} \in\left\{x_{a}, x_{b}, x_{c}\right\}$.

(a) If $f^{k-1}\left(p_{e}\right) \in \mathbf{I}$ then Theorem 1.4(a) holds.

(b) If $x_{0} \neq x_{a}$ and $f\left(x_{a}\right) \in \mathbf{I}$ then Theorem 1.4(a) holds. 
Proof. First suppose that $f^{k-1}\left(p_{e}\right) \in \mathbf{I}$, then we claim that the subinterval $M=$ $\left[p_{e}, f^{k-1}\left(p_{e}\right)\right]$ contains a fixed point $z$. Now we prove the claim. The interval $M \subset I$ contains points of $P$ (at least $p_{e}$ and $\left.f^{k-1}\left(p_{e}\right)\right)$. Since $P \cap \mathbf{O} \neq \varnothing$, and $f\left(f^{k-1}\left(p_{e}\right)\right)=p_{e}$, it follows that $M \rightarrow M$. So there is a fixed point in $M$, and the claim is proved. Notice that $z \neq x_{0}$ because $x_{0} \in\left\{x_{a}, x_{b}, x_{c}\right\}$. Therefore, from the monotonicity of $f$, the basic interval of $S \cap S^{\prime}$ containing $z \quad f$-covers itself. By Proposition 11.10 the result follows.

Finally suppose that $x_{0} \neq x_{a}$ and $f\left(x_{a}\right) \in \mathbf{I}$. Now we get that the interval $M=\left[p_{e}, x_{a}\right]$ contains a fixed point $z$. Since $x_{0} \in\left\{x_{b}, x_{c}\right\} \subset \mathbf{O}$, we have that $z \neq x_{0}$. Thus the proof follows as before.

Lemma 11.12. Let $f$ be the standard $\sigma$ map. If there exists a basic interval $J \in S^{\prime}, J \subset \mathbf{O}$ such that there are no basic intervals of $S^{\prime}$ different from $J$ $f$-covering $J$, and $f(0) \notin \operatorname{Int}(J)$, then Theorem 1.4(a) holds.

Proof. Since $f(0) \notin \operatorname{Int}(J), f$ is monotone on each basic interval of $S^{\prime}$, and there are no basic intervals of $S^{\prime}$ different from $J \quad f$-covering $J$, we can consider the map $g: \sigma \backslash \operatorname{Int}(J) \longrightarrow \sigma \backslash \operatorname{Int}(J)$ defined as $f$ restricted to $\sigma \backslash \operatorname{Int}(J)$. Clearly $g$ is well-defined and $g$ is either an I map or a $Y$ map. By Lemma $11.7 \operatorname{Int}(J)$ does not contain periodic points of period $n>1$. So $\operatorname{Per}(g)=\operatorname{Per}(f)$. Moreover $P$ is a periodic orbit for $g$. Hence from the Interval Theorem or the $\mathbf{Y}$ Theorem the result follows.

Lemma 11.13. Let $f$ be the standard $\sigma$ map. Suppose that each basic interval of $T$ is $f$-covered by some different basic interval of $T$ and $x_{0} \in\left\{x_{a}, x_{b}, x_{c}\right\}$. Denote by $I_{1}$ the basic interval of $S \cap S^{\prime}$ with $x_{0}$ as endpoint. Let $\{i, j\} \subset$ $\{1,2,3\}$ be with $i \neq j$ such that $L_{i} \cap L_{j}=\left\{x_{0}\right\}$. Assume that there is $J \in$ $S \cap S^{\prime} \backslash\left\{I_{1}\right\}$ such that one of the following subgraphs is satisfied:

(a) $J \rightarrow I_{1} \rightleftarrows L_{i} \leftarrow J$;

(b) $J \rightarrow L_{j} \rightarrow I_{1} \leftarrow J$ and $I_{1} \rightleftarrows L_{i}$.

Then Theorem 1.4(a) holds.

Proof. Clearly $I_{1} \cap L_{i}=\left\{x_{0}\right\}$. Then by Lemma 11.5 there is a path of length $l \leq k-1$ starting at one of the intervals $I_{1}$ or $L_{i}$ and ending at $J$. Suppose that the path starts at $I_{1}$. Then if (a) is satisfied we consider the loops $I_{1} \rightarrow \cdots \rightarrow$ $J \rightarrow I_{1}, I_{1} \rightarrow \cdots \rightarrow J \rightarrow L_{i} \rightarrow I_{1}$ and $I_{1} \rightleftarrows L_{i}$ of lengths $l+1 \leq k, l+2 \leq k+1$ and 2 respectively. If (b) is satisfied, then we consider the loops $I_{1} \rightarrow \cdots \rightarrow J \rightarrow$ $I_{1}, I_{1} \rightarrow \cdots \rightarrow J \rightarrow L_{j} \rightarrow I_{1}$ and $I_{1} \rightleftarrows L_{i}$ of lengths $l+1 \leq k, l+2 \leq k+1$ and 2 respectively. Suppose that the path starts at $L_{i}$. Then if (a) is satisfied we consider the loops $L_{i} \rightarrow \cdots \rightarrow J \rightarrow L_{i} \rightarrow I_{1} \rightarrow L_{i}, L_{i} \rightarrow \cdots \rightarrow J \rightarrow I_{1} \rightarrow L_{i}$ and $I_{1} \rightleftarrows L_{i}$ of lengths $l+3 \leq k+2, l+2 \leq k+1$ and 2 respectively. If (b) is satisfied, then we consider the loops $L_{i} \rightarrow \cdots \rightarrow J \rightarrow I_{1} \rightarrow L_{i}$, $L_{i} \rightarrow \cdots \rightarrow J \rightarrow L_{j} \rightarrow I_{1} \rightarrow L_{i}$ and $I_{1} \rightleftarrows L_{i}$ of lengths $l+2 \leq k+1, l+3 \leq k+2$ and 2 respectively. In all cases we obtain a non-repetitive loop of length $n$ for each $n \geq k+1$ containing $I_{1}$ and $J$. Notice that $0 \notin I_{1}$ and $x_{0} \notin J$. Then by the construction of this loop of length $n$ and Proposition 7.2 the result follows.

Lemma 11.14. Let $f$ be the standard $\sigma$ map. Suppose that $x_{0} \in\left\{x_{b}, x_{c}\right\}$. Denote by $I_{1}$ the basic interval of $S \cap S^{\prime}$ with $x_{0}$ as endpoint. Set $D_{1}=$ $\left[p_{c}, x_{a}\right] \subset \mathrm{I}$ and $D_{2}=\mathrm{Cl}\left(\mathbf{O} \backslash\left(B \cup C \cup I_{1}\right)\right)$. Suppose that $f\left(D_{1}\right) \subset D_{2}$ and $f\left(D_{2}\right) \subset D_{1}$. Then Theorem 1.4(a) holds. 
Proof. Notice that $P \subset D_{1} \cup D_{2}$. Since $f\left(D_{1}\right) \subset D_{2}$ and $f\left(D_{2}\right) \subset D_{1} \quad k$ must be even. Consider the interval map $g=\left.f^{2}\right|_{D_{1}}: D_{1} \longrightarrow D_{1}$. Clearly the points of $D_{1} \cap P$ have period $k / 2$ under $g$. From the Interval Theorem it follows that $S(k / 2) \subset \operatorname{Per}(g)$. Since $1 \in \operatorname{Per}(f)$, again from the fact that $f\left(D_{1}\right) \subset D_{2}$ and $f\left(D_{2}\right) \subset D_{1}$ we get that $S(k) \subset \operatorname{Per}(f)$.

Lemma 11.15. Let $f$ be the standard $\sigma$ map. Suppose that there exist two closed subintervals $K \subset \mathbf{I}$ and $N \subset \mathbf{O}$ such that $K$ has endpoints elements of $P \cup\left\{x_{0}\right\}$, and $K \rightleftarrows N$. If $k$ is odd then $n \in \operatorname{Per}(f)$ for each $n>k$ odd.

Proof. Since $K \subset I$ has endpoints elements of $P \cup\left\{x_{0}\right\}$, by Lemma 11.1 there is a loop of length $k$ odd containing $K$. This loop of length $k$ together with the loop $K \rightleftarrows N$ give us a non-repetitive loop of length $n$ for each $n>k$ odd. By the hypotheses $x_{0} \notin K \cap N$, then we obtain that $n \in \operatorname{Per}(f)$ for all $n>k$ odd.

\section{MAPS WITH $0 \in P \cup\left\{x_{0}\right\}$}

In this section we will prove Theorem $1.4(\mathrm{a})$ when $x_{0}=0$ or $0 \in P$. Then, under these assumptions $S=S^{\prime}=T$.

Proposition 12.1. Let $f$ be the standard $\sigma$ map. Suppose that $0 \in P \cup\left\{x_{0}\right\}$ and that there exists $J \in S^{\prime}$ such that the unique basic interval of $S^{\prime} f$-covering $J$ is itself. Then Theorem 1.4(a) holds.

Proof. We consider the two possibilities either $J \subset \mathbf{O}$ or $J \subset \mathbf{I}$. If $J \subset \mathbf{O}$, then we define the map $g=\left.f\right|_{\sigma \backslash \operatorname{Int}(J)}: \sigma \backslash \operatorname{Int}(J) \longrightarrow \sigma \backslash \operatorname{Int}(J)$. The map $g$ is well-defined because the unique basic interval of $S f$-covering $J$ is itself and $f$ is monotone on each basic interval of $S^{\prime}$. Clearly $k \in \operatorname{Per}(g)$ and since $f$ is monotone in $J, \operatorname{Per}(g)=\operatorname{Per}(f)$. Moreover $g$ is either an $\mathbf{I}$ map or an $\mathbf{Y}$ map. By the Interval Theorem or the $\mathbf{Y}$ Theorem, the result follows.

Suppose that $J \subset \mathbf{I}$. By Lemma $3.2 f$ has a fixed point $y \in J$. Denote by $D_{1}$ and $D_{2}$ the closures of the two connected components of $\sigma \backslash\{y\}$ such that $D_{1}$ is homeomorphic to $I$. Since $J \subset I$ and the unique interval of $S$ $f$-covering $J$ is itself, we obtain easily $f\left(D_{1}\right)=D_{2}$ and $f\left(D_{2}\right)=D_{1}$. By Lemma 11.3 Theorem 1.4(a) holds.

Remark 12.2. In what follows we can assume that if $0 \in P \cup\left\{x_{0}\right\}$, then each basic interval of $S$ is $f$-covered by some basic interval of $S$ different from itself.

Lemma 12.3. Let $f$ be the standard $\sigma$ map. Suppose that $0 \in P \cup\left\{x_{0}\right\}$. If there is a basic interval $J$-covering itself, then Theorem 1.4(a) follows.

Proof. By Remark 12.2 there exists a basic interval $M \in S$ different from $J$ such that $M \rightarrow J$. By Lemma 11.4 there is a non-repetitive loop of length $n$ for each $n \geq k+1$ containing $J$. We claim that at least one basic interval of this loop does not contain $x_{0}$. In fact, if $x_{0} \in J$, by the monotonicity of $f$ on each basic interval of $S^{\prime}$, we have that $f(J)$ does not contain intervals of $S$ different from $J$ with $x_{0}$ as endpoint. By Proposition 7.1 we get $B(k) \subset \operatorname{Per}(f)$ and the result follows.

Proposition 12.4. Let $f$ be the standard $\sigma$ map. If $x_{0}=0$ then Theorem 1.4(a) holds. 
Proof. If there is a basic interval $J f$-covering itself, by Lemma 12.3, the result follows. So we can suppose that each basic interval does not $f$-cover itself. By Remark 9.6, there exists a closed subinterval $K$ of $\sigma$ such that $K$ $f$-covers $O$. We can assume that $K$ has endpoints elements of $P \cup\left\{\ddot{x}_{0}\right\}$. Since $f(0)=0$ and there are no basic intervals $f$-covering themselves, we get that $K \subset \mathbf{I}$. If $f^{k-1}\left(p_{e}\right) \in \mathbf{I}$, then the interval $\left[p_{e}, f^{k-1}\left(p_{e}\right)\right]$ contains a fixed point $z \neq 0$. Therefore, by the monotonicity of $f$, the basic interval of $S$ containing $z \quad f$-covers itself, in contradiction with the fact that there are no basic intervals $f$-covering themselves. Hence $f^{k-1}\left(p_{e}\right) \in \mathbf{O}$. So there are two closed subintervals $M, N \subset \mathbf{O} f$-covering $K$ in the hypotheses of Lemma 11.6. So the result follows.

From now on we can assume that $x_{0} \neq 0$.

Proposition 12.5. Let $f$ be the standard $\sigma$ map. Suppose that $0 \in P$, then Theorem 1.4(a) holds.

Proof. If there is some basic interval $f$-covering itself, from Lemma 12.3 the result follows. So we can assume that each basic interval does not $f$-cover itself. Denote by $I_{1}, I_{2}$ the two basic intervals having $x_{0}$ as endpoint. We have $I_{1} \rightleftarrows I_{2}$. Notice that a basic interval $J \notin\left\{I_{1}, I_{2}\right\} \quad f$-covers $I_{1}$ if and only if $f$-covers $I_{2}$.

First suppose that there are no basic intervals $f$-covering $I_{1}$ and $I_{2}$. If $x_{0} \in$ $I$, then denote by $D_{1}$ and $D_{2}$ the closures of the two connected components of $\sigma \backslash\left\{x_{0}\right\}$ such that $D_{1}$ is homeomorphic to $I$. Hence $f\left(D_{1}\right)=D_{2}$ and $f\left(D_{2}\right)=D_{1}$. From Lemma 11.3 the result follows. If $x_{0} \in \mathbf{O}$, then consider either the $I$ map or the $Y$ map $g$ defined by $f$ restricted to $\sigma \backslash \operatorname{Int}\left(I_{1} \cup I_{2}\right)$. Clearly $k \in \operatorname{Per}(g)$ and $\operatorname{Per}(g)=\operatorname{Per}(f)$. So by the Interval Theorem or the $Y$ Theorem the result follows.

Finally suppose that there is a basic interval $J$ such that $I_{1} \leftarrow J \rightarrow I_{2}$. By Lemma 11.5 there is a path of length $l \leq k-1$ starting at one of the intervals $I_{1}$ or $I_{2}$ and ending at $J$. By construction, this path together with $I_{1} \leftarrow J \rightarrow I_{2}$ and $I_{1} \rightleftarrows I_{2}$ give us a non-repetitive loop of length $n$ for each $n \geq k+1$. Since $x_{0} \notin J$ from Proposition 7.1 it follows that $B(k) \subset \operatorname{Per}(f)$.

From now on we can asssume that $0 \notin P \cup\left\{x_{0}\right\}$.

Remark 12.6. Suppose that there is a basic interval $J=\left[0, x_{i}\right] \in S^{\prime}$ for some $x_{i} \in P \cup\left\{x_{0}\right\}$ such that $f(0)=f\left(x_{i}\right)$. Notice that from the monotonicity of $f$ on each basic interval of $S^{\prime}, f$ is constant in $J$. Then we consider the new topological space $\sigma^{\prime}$ homeomorphic to $\sigma$ obtained by shrinking the interval $J$ to the point 0 , and define the $\sigma^{\prime}$ map $g$ in the natural way as the $\sigma$ map $\left.f\right|_{\sigma \backslash \operatorname{Int}(J)}$. So $P$ is a periodic orbit of period $k$ for $g$ and clearly $\operatorname{Per}(g)=\operatorname{Per}(f)$. Now $0 \in P \cup\left\{x_{0}\right\}$. From Propositions 12.4 and 12.5 the result follows. Hence from now on we can assume that $0 \notin P \cup\left\{x_{0}\right\}$ and $f(0) \notin\left\{f\left(x_{a}\right), f\left(x_{b}\right), f\left(x_{c}\right)\right\}$.

\section{MaPS WITh $f(0) \notin \operatorname{Int}(A \cup B \cup C)$}

In this section we prove Theorem 1.4(a) when $f(0) \notin \operatorname{Int}(A \cup B \cup C)$. Notice that from now on 0 can be a periodic or non-periodic point, and if it is periodic it does not belong to $P$. 
Proposition 13.1. Let $f$ be the standard $\sigma$ map. Suppose that $f(0) \notin \operatorname{Int}(A \cup$ $B \cup C)$ and $x_{0} \notin\left\{x_{a}, x_{b}, x_{c}\right\}$. Then Theorem 1.4(a) holds.

Proof. Let $I_{1}, I_{2}$ be the two basic intervals of $S$ with $x_{0}$ as endpoint. Such intervals exist in virtue of Remark 6.4. Moreover $I_{1}, I_{2} \in S \cap S^{\prime}$ because $x_{0} \notin\left\{x_{a}, x_{b}, x_{c}\right\}$. By hypotheses $f(0) \notin \operatorname{Int}(A \cup B \cup C)$, hence $f(0) \notin$ $\operatorname{Int}\left(L_{1} \cup L_{2} \cup L_{3}\right)$. Therefore from Remark 11.9 it follows that each basic interval of $T$ is $f$-covered by some basic interval different from itself. If $I_{i} \rightarrow I_{i}$ for some $i=1,2$, by Proposition 11.10 the result follows. So assume that $I_{i}$ does not $f$-cover itself for $i=1,2$. Then we get the loop $I_{1} \rightleftarrows I_{2}$.

First suppose that $x_{0} \in \mathbf{I}$. Denote by $D_{1}$ and $D_{2}$ the closure of the two connected componets of $\sigma \backslash\left\{x_{0}\right\}$ such that $D_{1}$ is homeomorphic to I. If $f\left(D_{1}\right)=D_{2}$ and $f\left(D_{2}\right)=D_{1}$ by Lemma 11.3 the result follows. Otherwise, by Remark 10.2, there exists $y \in D_{i} \cap P$ such that $f(y) \in D_{i} \cap P$ for some $i=1,2$. Then since $I_{1} \rightleftarrows I_{2}$, by the continuity of $f$, there exists a basic interval $J \in S f$-covering $I_{1}$ and $I_{2}$. By Lemma 10.7 , we can assume that $J \in T$. From the monotonicity of $f$ on $I_{1}$ and $I_{2}$ we get that $J \notin\left\{I_{1}, I_{2}\right\}$.

Now suppose $x_{0} \in \mathbf{O}$. If there are no basic intervals of $S$ different from $I_{1}$ and $I_{2} f$-covering $I_{1}$ or $I_{2}$, then we consider the $Y$ map $g$ defined by $f$ restricted to $\sigma \backslash \operatorname{Int}\left(I_{1} \cup I_{2}\right)$. Clearly $g$ is well-defined, $k \in \operatorname{Per}(g)$ and $\operatorname{Per}(g) \subset \operatorname{Per}(f)$. From the $\mathbf{Y}$ Theorem the result follows. So suppose that there exists $J \in S, J \notin\left\{I_{1}, I_{2}\right\} \quad f$-covering $I_{1}$ or $I_{2}$. Since $I_{1} \cap I_{2}=\left\{x_{0}\right\}$, if $f(0) \notin I_{1} \cup I_{2}$ then $I_{1} \leftarrow J \rightarrow I_{2}$; if $f(0) \in I_{1} \cup I_{2}$ then, by Remark 10.2 we obtain that $I_{1} \leftarrow J \rightarrow I_{2}$. Furthermore, from Lemma 10.7, we can assume that $J \in T$.

Finally we consider the two possibilities either $x_{0} \in \mathbf{I}$ or $x_{0} \in \mathbf{O}$ simultaneously. Since we are in the hypotheses of Lemma 11.5, there is a path of length $l \leq k-1$ starting at one of the intervals $I_{1}, I_{2}$ and ending at $J$. Without loss of generality we can assume that the loop starts at $I_{1}$. So we consider the loops $I_{1} \rightarrow \cdots \rightarrow J \rightarrow I_{1}$ and $I_{1} \rightarrow \cdots \rightarrow J \rightarrow I_{2} \rightarrow I_{1}$ of lengths $l+1 \leq k$ and $l+2 \leq k+1$ respectively. By construction (see the proof of Lemma 11.5), these loops are formed by different intervals. The two above loops together with the loop $I_{1} \rightleftarrows I_{2}$ give us a loop of length $n$ for each $n \geq k+1$ containing $J$ and also containing $I_{1}$ or $I_{2}$. Since $J \notin\left\{I_{1}, I_{2}\right\}$, we have $x_{0} \notin J$. Furthermore $0 \notin I_{1} \cup I_{2}$. By construction of the loop of length $n$, the associated sequence of $W_{i}$ 's in Proposition 7.2 is non-repetitive. Hence from Proposition 7.2 we get that $B(k) \subset \operatorname{Per}(f)$.

Proposition 13.2. Let $f$ be the standard $\sigma$ map. Suppose that $f(0) \notin$ $\operatorname{Int}(A \cup B \cup C)$ and $x_{0} \in\left\{x_{a}, x_{b}, x_{c}\right\}$. Then Theorem 1.4(a) holds.

Proof. Denote by $I_{1}=\left[x_{0}, x_{1}\right]$ the basic interval of $S \cap S^{\prime}$ with $x_{0}$ as endpoint. By hypotheses $f(0) \notin \operatorname{Int}(A \cup B \cup C)$, hence $f(0) \notin \operatorname{Int}\left(L_{1} \cup L_{2} \cup L_{3}\right)$. Therefore from Remark 11.9 it follows that each basic interval of $T$ is $f$-covered by some basic interval different from itself. By Proposition 11.10 we can assume that each $J \in S \cap S^{\prime}$ does not $f$-cover itself. In particular, $I_{1}$ does not $f$-cover itself. From Corollary 11.11(a) we can suppose that $f^{k-1}\left(p_{e}\right) \in \mathbf{O}$ and $f\left(x_{a}\right) \in \mathbf{O}$.

If $x_{0}=x_{a}$, by Remark 6.2, we have $f(0) \in \mathbf{I}$. Since $I_{1}$ does not $f$-cover itself we get that $I_{1} \rightarrow A$. Therefore either $I_{1} \rightarrow L_{1}$ or $I_{1} \rightarrow L_{2}$. Without loss of generality we can assume that $I_{1} \rightarrow L_{1}$. Denote by $D_{1}$ and $D_{2}$ the closures of the two connected components of $\sigma \backslash\left\{x_{0}\right\}$ such that $D_{1}$ is homeomorphic 
to I. If $f\left(D_{1}\right)=D_{2}$ and $f\left(D_{2}\right)=D_{1}$, by Lemma 11.3 the result follows. Otherwise, since $f(0) \notin \operatorname{Int}(A \cup B \cup C), x_{0}=x_{a}$ and from Remark 12.6 $f(0) \neq x_{a}$, it follows that there exists $z \in P \cap D_{i}$ such that $f(z) \in P \cap D_{i}$ for some $i \in\{1,2\}$. By continuity of $f$, there exists $J \in S \cap S^{\prime}$ such that $I_{1} \leftarrow J \rightarrow A$ and so either $I_{1} \leftarrow J \rightarrow L_{1}$ or $I_{1} \leftarrow J \rightarrow L_{2}$.

By symmetry we only need to consider the following three cases.

Case 1: $x_{0}=x_{a}$ and $f(0) \notin \operatorname{Int}\left(I_{1}\right)$. Since $f(0) \in \mathbf{I}$, we get $A \rightarrow I_{1}$, and so $L_{1} \rightarrow I_{1}$ and $L_{2} \rightarrow I_{1}$. Then we obtain the loop $I_{1} \rightleftarrows L_{1}$. Since $I_{1} \cap L_{1}=\left\{x_{0}\right\}$, by Lemma 11.5 there is a path of length $l \leq k-1$ starting at one of the intervals $I_{1}$ or $L_{1}$ and ending at $J$.

First suppose that $I_{1} \leftarrow J \rightarrow L_{1}$. Then we obtain the loops either $I_{1} \rightleftarrows L_{1}$, $I_{1} \rightarrow \cdots \rightarrow J \rightarrow I_{1}$ and $I_{1} \rightarrow \cdots \rightarrow J \rightarrow L_{1} \rightarrow I_{1}$, or $I_{1} \rightleftarrows L_{1}, L_{1} \rightarrow \cdots \rightarrow$ $J \rightarrow L_{1}$ and $L_{1} \rightarrow \cdots \rightarrow J \rightarrow I_{1} \rightarrow L_{1}$ of lengths $2, l+1 \leq k$ and $l+2 \leq k+1$ respectively. Then we get a loop of length $n$ for each $n \geq k+1$ containing $J$. Notice that $\left\{x_{0}, 0\right\} \cap J=\varnothing$. By construction of the loop of length $n$, the associated sequence of $W_{i}$ 's of Proposition 7.2 is non-repetitive. Hence $B(k) \subset \operatorname{Per}(f)$.

Now suppose that $I_{1} \leftarrow J \rightarrow L_{2}$. By Lemma 11.5 there is a path of length $l \leq k-1$ starting at one of the intervals $I_{1}$ or $L_{1}$ and ending at $J$. If the path starts at $I_{1}$, then we get the loops $I_{1} \rightleftarrows L_{1}, I_{1} \rightarrow \cdots \rightarrow J \rightarrow I_{1}$ and $I_{1} \rightarrow \cdots \rightarrow J \rightarrow L_{2} \rightarrow I_{1}$ of lengths $2, l+1 \leq k$ and $l+2 \leq k+1$ respectively. If the path starts at $L_{1}$, then we obtain the loops $I_{1} \rightleftarrows L_{1}$, $L_{1} \rightarrow \cdots \rightarrow J \rightarrow I_{1} \rightarrow L_{1}$ and $L_{1} \rightarrow \cdots \rightarrow J \rightarrow L_{2} \rightarrow I_{1} \rightarrow L_{1}$ of lengths $2, l+2 \leq k+1$ and $l+3 \leq k+2$ respectively. As above, Proposition 7.2 allows us to prove that $B(k) \subset \operatorname{Per}(f)$.

Case 2: $x_{0}=x_{a}$ and $f(0) \in \operatorname{Int}\left(I_{1}\right)$.

Suppose that $L_{1} \rightarrow L_{1}$. By Lemma 11.4 there is a loop of length $n$ for each $n \geq k+1$ containing $L_{1}$. Since there exists $J \in S \cap S^{\prime}$ such that $J \rightarrow I_{1} \rightarrow L_{1}$, from Lemma 11.4 we can assume that it contains the path $J \rightarrow I_{1} \rightarrow L_{1}$. Since $\left\{x_{0}, 0\right\} \cap J=\varnothing$, by the above construction the loop verifies the hypotheses of Proposition 7.2. So $B(k) \subset \operatorname{Per}(f)$. Hence in the rest of the proof of Case 2 we can assume that $L_{1}$ does not $f$-cover $L_{1}$.

Subcase (i). $L_{1} \rightarrow I_{1}$. Then, since $f(0) \in \operatorname{Int}\left(I_{1}\right), f\left(x_{b}\right) \in \mathbf{I}$. We have the loop $I_{1} \rightleftarrows L_{1}$. Suppose that $I_{1} \leftarrow J \rightarrow L_{1}$. So by Lemma 11.5 there is a path of length $l \leq k-1$ starting at one of the intervals $I_{1}$ or $L_{1}$ and ending at $J$. This path together with the loop $I_{1} \rightleftarrows L_{1}$ give us a loop of length $n$ for each $n \geq k+1$. Notice that $\left\{x_{0}, 0\right\} \cap J=\varnothing$. By construction the above loop satisfies the hypotheses of Proposition 7.2 and consequently $B(k) \subset \operatorname{Per}(f)$.

So we can assume that there are no basic intervals of $S \cap S^{\prime} f$-covering $I_{1} \cup L_{1}$. Therefore we have $I_{1} \leftarrow J \rightarrow L_{2}$. In particular $L_{2} \in T$. Suppose that $L_{2} \rightarrow I_{1}$. Then by Lemma 11.5 we obtain the loops either $I_{1} \rightleftarrows L_{1}$, $I_{1} \rightarrow \cdots \rightarrow J \rightarrow I_{1}$ and $I_{1} \rightarrow \cdots \rightarrow J \rightarrow L_{2} \rightarrow I_{1}$, or $I_{1} \rightleftarrows L_{1}, L_{1} \rightarrow \cdots \rightarrow$ $J \rightarrow I_{1} \rightarrow L_{1}$ and $L_{1} \rightarrow \cdots \rightarrow J \rightarrow L_{2} \rightarrow I_{1} \rightarrow L_{1}$ of lengths either $2, l+1 \leq k$ and $l+2 \leq k+1$, or $2, l+2 \leq k+1$ and $l+3 \leq k+2$ respectively. Since $\left\{x_{0}, 0\right\} \cap J=\varnothing$ and by construction from these loops we can obtain a loop of length $n$ for each $n \geq k+1$ in the hypotheses of Proposition 7.2. So the result follows.

Hence we can suppose that $L_{2}$ does not $f$-cover $I_{1}$. Therefore $f\left(x_{c}\right) \in \mathbf{O}$ and either $C \rightarrow L_{1}$ or $C \rightarrow L_{2}$ because $f(0) \in \operatorname{Int}\left(I_{1}\right)$. Suppose that $C \rightarrow L_{2}$, 


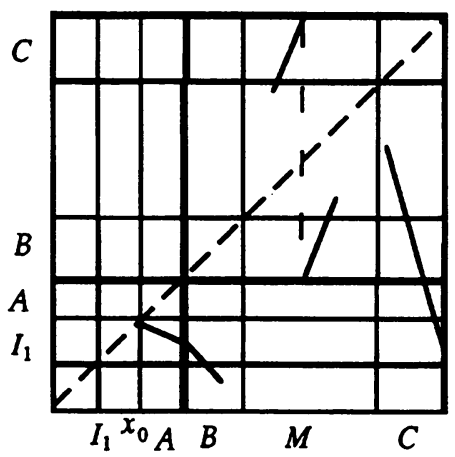

Figure 13.1. In Subcase (i) there is $M \in S \cap S^{\prime}, M \subset \mathbf{O}$ such that $M \rightarrow L_{3}$.

then $L_{2} f$-covers itself. Consider the path $J \rightarrow L_{2}$. By Lemma 11.4 there exists a non-repetitive loop of length $n$ for each $n \geq k+1$ containing $L_{2}$. By construction of the above loop we can assume that it contains the path $J \rightarrow L_{2}$. Since $\left\{x_{0}, 0\right\} \cap J=\varnothing$ and the associated sequence of the $W_{i}^{\prime}$ s of Proposition 7.2 is non-repetitive, we get that $B(k) \subset \operatorname{Per}(f)$. Hence we can assume that $C$ does not $f$-cover $L_{2}$. Therefore $C \rightarrow L_{1}$ and so $L_{3} \rightarrow L_{1} \cup I_{1}$ because $f\left(x_{b}\right) \in \mathbf{I}$. Since $f\left(x_{b}\right) \in \mathbf{I}, f\left(x_{c}\right) \in \mathbf{O}$, each basic interval of $S \cap S^{\prime}$ does not $f$-cover itself, there are no basic intervals of $S \cap S^{\prime} \quad f$-covering $L_{1} \cup I_{1}$ and from the continuity of $f$, it follows that there exists $M \in S \cap S^{\prime}, M \subset \mathbf{O}$ such that $M \rightarrow L_{3}$ (see Figure 13.1). In particular $L_{3} \in T$. Therefore consider the paths $I_{1} \leftarrow L_{3} \rightarrow L_{1} \rightleftarrows I_{1}$. By Lemma 11.5 there is a path of length $l \leq k-1$ starting at one of the intervals $I_{1}$ or $L_{1}$ and ending at $L_{3}$. So we obtain either the loops $I_{1} \rightleftarrows L_{1}, I_{1} \rightarrow \cdots \rightarrow L_{3} \rightarrow I_{1}$ and $I_{1} \rightarrow \cdots \rightarrow L_{3} \rightarrow L_{1} \rightarrow I_{1}$, or $I_{1} \rightleftarrows L_{1}, L_{1} \rightarrow \cdots \rightarrow L_{3} \rightarrow L_{1}$ and $L_{1} \rightarrow \cdots \rightarrow L_{3} \rightarrow I_{1} \rightarrow L_{1}$ of lengths $2, l+1 \leq k$ and $l+2 \leq k+1$ respectively. So we obtain a loop of length $n$ for each $n \geq k+1$ containing $I_{1}$ and $L_{3}$. By construction of this loop and from the facts that $0 \notin I_{1}$ and $x_{0} \notin L_{3}$, the associated sequence of $W_{i}^{\prime}$ s of Proposition 7.2 is non-repetitive. Hence $B(k) \subset \operatorname{Per}(f)$.

Subcase (ii). $L_{1} \nrightarrow I_{1}$. So, since $x_{b} \in P$ we have that $f\left(x_{b}\right) \in \mathbf{O}$. By Remark 10.2 we get that $f\left(x_{c}\right) \in \mathbf{I}$. From the facts that there exists a closed subinterval $K \subset \boldsymbol{\sigma} f$-covering $O$ (see Remark 9.6), each basic interval of $\left(S \cap S^{\prime}\right) \cup\left\{L_{1}\right\}$ does not $f$-cover itself, $f\left(x_{c}\right) \in \mathbf{I}$ and $f(0) \in \mathbf{I}$, it follows that $K \subset I$ (see Figure 13.2). Moreover we can assume that $K$ has endpoints elements of $P \cup\left\{x_{0}\right\}$.

Since $f\left(x_{b}\right) \in \mathbf{O}$ and $f^{k-1}\left(p_{e}\right) \in \mathbf{O}$, there is a closed subinterval $M \subset \mathbf{O}$ such that $M \rightleftarrows K$.

Let $p_{0} \in I_{1}$ be such that $f\left(p_{0}\right)=0$. Consider $K^{\prime} \subset K$ a minimal closed subinterval $f$-covering $\mathbf{O}$. Then $K^{\prime} \subset\left[p_{e}, p_{0}\right]$. Since $f^{k-1}\left(p_{e}\right) \in \mathbf{O}$, there are two minimal closed subintervals $M^{\prime}, N^{\prime} \subset \mathbf{O} \backslash B \quad f$-covering $\left[p_{e}, f(0)\right]$ such that $M^{\prime}$ and $N^{\prime}$ have pairwise disjoint interiors. We have $M^{\prime} \leftarrow K^{\prime} \rightarrow N^{\prime}$.

First suppose that $p_{0} \in\left[p_{e}, f(0)\right]$. Then, since $K^{\prime} \subset\left[p_{e}, p_{0}\right]$, we get that $M^{\prime} \rightleftarrows K \rightleftarrows N^{\prime}$. Thus we obtain a non-repetitive loop of length $n$ for each $n$ even. Since $x_{0} \notin M^{\prime} \cup N^{\prime} \cup K^{\prime}$ and $0 \notin K^{\prime}$ we get that $2 \mathbb{N} \subset \operatorname{Per}(f)$. If $k$ is even, then we are done because $S(k) \subset \operatorname{Per}(f)$. So we suppose that $k$ is odd. 


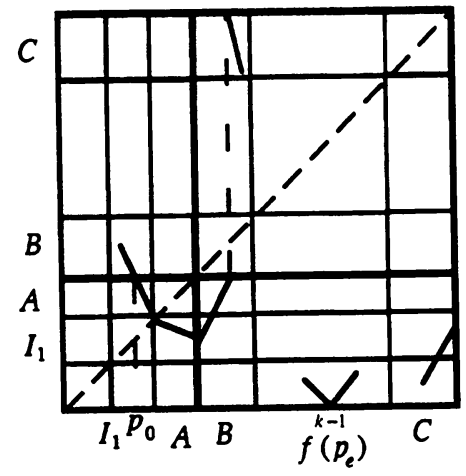

FIGURE 13.2. In Subcase (ii) the graph of $f$ has only one fixed point, $x_{0}$.

Since $x_{0} \notin M_{1}$, from Lemma 11.15 we get that $n \in \operatorname{Per}(f)$ for each $n>k$ odd. Therefore $S(k) \subset \operatorname{Per}(f)$ and the result follows.

Finally suppose that $p_{0} \notin\left[p_{e}, f(0)\right]$. Then we have $\left[p_{0}, 0\right] \rightarrow\left[p_{0}, 0\right] \rightarrow$ $\left[f(0), p_{0}\right]$ (see Figure 13.3) and $B \rightarrow\left[p_{0}, 0\right]$ (see Figure 13.2). Assume that $f^{2}(0) \notin \operatorname{Int}(B)$. Then, since $I_{1} \rightarrow L_{1}$, again from Figure 13.2 we have that $\left[f(0), p_{0}\right] \rightarrow B$. So the loops $\left[p_{0}, 0\right] \rightarrow\left[p_{0}, 0\right] \rightarrow\left[f(0), p_{0}\right] \rightarrow B \rightarrow\left[p_{0}, 0\right]$ give us a non-repetitive loop of length $n$ for each $n \geq k+1$. Hence $B(k) \subset$ $\operatorname{Per}(f)$. Now assume that $f^{2}(0) \in \operatorname{Int}(B)$. Then, since $M^{\prime} \cup N^{\prime} \subset \mathbf{O} \backslash B$ we obtain $M^{\prime} \rightleftarrows K^{\prime} \rightleftarrows N^{\prime}$. Notice that $x_{0} \notin M^{\prime} \cup N^{\prime} \cup K^{\prime}$. By the above arguments $S(k) \subset \operatorname{Per}(f)$.

Case 3: $x_{0}=x_{b}$. From Corollary 11.11(b) we can suppose that $f\left(x_{a}\right) \in \mathbf{O}$. By Remark 6.2 we have that $f(0) \in \mathbf{O}$. Since $I_{1} \rightarrow I_{1}$, we get $I_{1} \rightarrow B$. So either $I_{1} \rightarrow B \cup A=L_{1}$ or $I_{1} \rightarrow B \cup C=L_{3}$.

Subcase (i). Suppose that $C \rightarrow C$. Therefore, since $f(0) \notin \operatorname{Int}(A \cup B \cup C)$, we obtain either $C \rightarrow A$ or $C \rightarrow B$. Consequently either $C \rightarrow L_{2}$ or $C \rightarrow L_{3}$. Therefore at least one of the following statements is satisfied:

(a) $C \rightarrow L_{2}$ and $L_{3} \rightarrow L_{2} \rightarrow L_{2}$,

(b) $C \rightarrow L_{3}$ and $L_{2} \rightarrow L_{3} \rightarrow L_{3}$.

First assume that (a) holds. If there exists $J \in S \cap S^{\prime}$ such that $J \rightarrow L_{2}$ or $J \rightarrow L_{3}$, then we consider the path $J \rightarrow L_{2} \rightarrow L_{2}$ or $J \rightarrow L_{3} \rightarrow L_{2} \rightarrow$ $L_{2}$. Given that each basic interval of $T$ is $f$-covered by some different basic interval, by Lemma 11.4 there is a non-repetitive loop of length $n$ for each $n \geq k+1$ containing $L_{2}$. Furthermore we can assume that the above loop of length $n$ also contains $J$. Clearly $0 \notin J$ and $x_{0} \notin L_{2}$. By Proposition 7.2 the result follows.

Therefore we suppose that for each $J \in S \cap S^{\prime}, J \nrightarrow L_{2}$ and $J \nrightarrow L_{3}$. Consequently, since either $I_{1} \rightarrow L_{1}$ or $I_{1} \rightarrow L_{3}$, we get that $I_{1} \rightarrow L_{1}$. Since $f(0) \notin \operatorname{Int}(C)$, from Lemma 11.12 we can assume that there is a basic interval $J_{0} \in S^{\prime} f$-covering $C$. Taking into account that $C \subset L_{2} \cap L_{3}$, and that for each $J \in S \cap S^{\prime}, J \nrightarrow L_{2}$ and $J \nrightarrow L_{3}$, we get that $J_{0} \in\{A, B\}$. In particular $L_{1} \rightarrow C$. Hence $L_{1} \rightarrow L_{2}$ or $L_{1} \rightarrow L_{3}$. Then consider the path $I_{1} \rightarrow L_{1} \rightarrow L_{2} \rightarrow L_{2}$ or $I_{1} \rightarrow L_{1} \rightarrow L_{3} \rightarrow L_{2} \rightarrow L_{2}$. By Lemma 11.4 there is a non-repetitive loop of length $n$ for each $n \geq k+1$ containing $L_{2}$ and $I_{1}$. Notice that $0 \notin I_{1}$ and $x_{0} \notin L_{2}$. Then by Proposition 7.2 the result follows. 


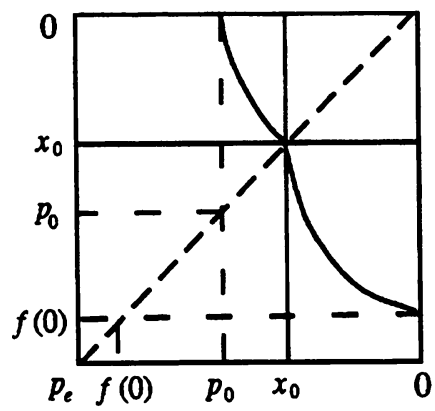

FigURE 13.3. The graph of $f$ when $p_{0} \notin\left[p_{e}, f(0)\right]$.

Finally suppose that (b) holds. Since $C \rightarrow C \cup B$, by the monotonicity of $f$ on $C$ we get that $f\left(x_{c}\right) \in \mathbf{O}$. Notice that either $I_{1} \rightarrow L_{1}$ or $I_{1} \rightarrow L_{3}$, and so $I_{1} \nrightarrow L_{2}$. If there is a basic interval $J \in S \cap S^{\prime} \backslash\left\{I_{1}\right\}$ such that either $J \rightarrow L_{2}$ or $J \rightarrow L_{3}$, then we consider either the path $J \rightarrow L_{2} \rightarrow L_{3} \rightarrow L_{3}$ or the path $J \rightarrow L_{3} \rightarrow L_{3}$. Clearly $\left\{0, x_{0}\right\} \cap J=\varnothing$. Therefore by Lemma 11.4 and Proposition 7.2 the result follows.

Thus we can assume that $L_{2}$ is not $f$-covered by any basic interval of $S \cap S^{\prime}$ and $L_{3}$ is not $f$-covered by any basic interval of $S \cap S^{\prime} \backslash\left\{I_{1}\right\}$. If $I_{1} \rightarrow L_{3}$, from the fact that each basic interval of $S \cap S^{\prime}$ does not $f$-cover itself and by the continuity of $f$, we have that there exists $J \in S \cap S^{\prime} \backslash\left\{I_{1}\right\}, J \subset \mathbf{O}$ $f$-covering $C$ (see Figure 13.4). Consequently either $J \rightarrow L_{2}$ or $J \rightarrow L_{3}$ in contradiction with the assumptions. Hence we can suppose that $I_{1} \rightarrow L_{3}$. Therefore $I_{1} \rightarrow L_{1}$. In particular $I_{1} \rightarrow C$. By Lemma 11.12 we can assume that there is $J_{0} \in S^{\prime} \quad f$-covering $C$. Since for each $J \in S \cap S^{\prime}, J \nrightarrow L_{2}$ and $J \nrightarrow L_{3}$, we obtain that $J_{0} \in\{A, B\}$. Thus $L_{1} \rightarrow L_{2}$ or $L_{1} \rightarrow L_{3}$. From the facts that $f^{k-1}\left(p_{e}\right) \in \mathbf{O}, f\left(x_{c}\right) \in \mathbf{O}$ and by the continuity of $f$, it follows that there is $J \in S \cap S^{\prime}, J \neq I_{1}$ such that $J \rightarrow A$. Moreover either $J \rightarrow L_{1}$ or $J \rightarrow L_{2}$. By the above assumptions we have that $J \rightarrow L_{1}$. Notice that $\left\{0, x_{0}\right\} \cap J=\varnothing$. Consider the loops $J \rightarrow L_{1} \rightarrow L_{2} \rightarrow L_{3} \rightarrow L_{3}$ or $J \rightarrow L_{1} \rightarrow L_{3} \rightarrow L_{3}$. From Lemma 11.4 and Proposition 7.2 the result follows.

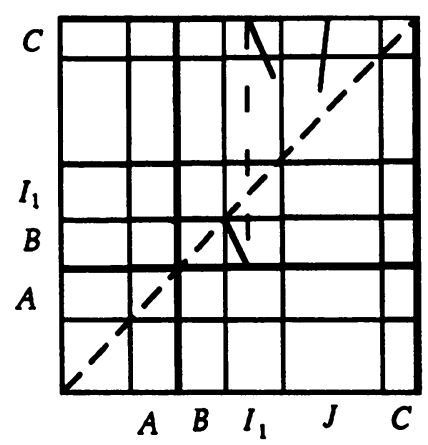

FIGURE 13.4. The graph of $f$ cannot cross the diagonal in $\mathbf{O} \backslash(B \cup C)$. 
Subcase (ii). Suppose that $C \nrightarrow C$. First assume that $B \rightarrow B$. Since $f(0) \in \mathbf{O}, f(0) \notin \operatorname{Int}(A \cup B \cup C)$ and $f$ is monotone in $B$, it follows that $B \rightarrow B \cup C=L_{3}$, which implies that $L_{1} \rightarrow L_{3} \rightarrow L_{3}$. If there is $J \in S \cap S^{\prime} \backslash\left\{I_{1}\right\}$ such that either $J \rightarrow L_{1}$ or $J \rightarrow L_{3}$, then we consider either the path $J \rightarrow$ $L_{1} \rightarrow L_{3} \rightarrow L_{3}$ or the path $J \rightarrow L_{3} \rightarrow L_{3}$. Clearly $\left\{0, x_{0}\right\} \cap J=\varnothing$. So by Lemma 11.4 and Proposition 7.2 the result follows. Hence we can assume that for each $J \in S \cap S^{\prime} \backslash\left\{I_{1}\right\}, J \nrightarrow L_{1}$ and $J \nrightarrow L_{3}$. So we claim that $f\left(x_{c}\right) \in \mathbf{I}$. Otherwise, $f\left(x_{c}\right) \in \mathbf{O}$, and since any basic interval of $S \cap S^{\prime}$ cannot $f$-cover itself, $f^{k-1}\left(p_{e}\right) \in \mathbf{O}$ and by the continuity of $f$, there exists $J \in S \cap S^{\prime} \backslash\left\{I_{1}\right\}$ such that $J \rightarrow B$. Therefore either $J \rightarrow A \cup B=L_{1}$ or $J \rightarrow B \cup C=L_{3}$, in contradiction with the assumptions. Then the claim is proved.

Now, from the facts that $f(0) \in \mathbf{O}, f\left(x_{c}\right) \in \mathbf{I}, f(0) \notin \operatorname{Int}(A \cup B \cup C)$ and $C \nrightarrow C$, we get that $C \rightarrow L_{1}$. Therefore $L_{2} \rightarrow L_{1}$ and $L_{3} \rightarrow L_{1}$. Then we have $L_{2} \rightarrow L_{1} \rightleftarrows L_{3} \rightarrow L_{3}$. If there is $J \in S \cap S^{\prime} \backslash\left\{I_{1}\right\}$ such that $J \rightarrow L_{2}$, then we consider the path $J \rightarrow L_{2} \rightarrow L_{1} \rightarrow L_{3} \rightarrow L_{3}$. Since $\left\{x_{0}, 0\right\} \cap J=\varnothing$, from Lemma 11.4 and Proposition 7.2 the result holds. Hence we can assume that $J \nrightarrow L_{2}$, for each $J \in S \cap S^{\prime} \backslash\left\{I_{1}\right\}$. Therefore, since $f^{k-1}\left(p_{e}\right) \in \mathbf{O}$ and by the continuity it follows that $f\left(x_{1}\right) \in \mathbf{O}$ (otherwise there is $J \in S \cap S^{\prime} \backslash\left\{I_{1}\right\}$ $f$-covering $L_{1}$ or $L_{2}$ or $\left.L_{3}\right)$. Consequently, if we take $D_{1}=\left[p_{e}, x_{a}\right]$ and $D_{2}=\mathrm{Cl}\left(\mathbf{O} \backslash\left(B \cup C \cup I_{1}\right)\right)$, we obtain that $f\left(D_{1}\right) \subset D_{2}$ and $f\left(D_{2}\right) \subset D_{1}$ (see Figure 13.5). Thus by Lemma 11.14 the result follows.

So in the rest of this proof we can suppose that $B \nrightarrow B$. Since $f\left(x_{a}\right) \in \mathbf{O}$ and $f(0) \in \mathbf{O}$ it follows that $A \nrightarrow A$.

First assume that $f(0) \notin \operatorname{Int}\left(I_{1}\right)$. Therefore, since $B \nrightarrow B$ by Remark 12.6 $f(0) \neq x_{0}$ and so $B \rightarrow I_{1}$. Hence we have $L_{1} \rightarrow I_{1} \leftarrow L_{3}$. Moreover, since either $I_{1} \rightarrow L_{1}$ or $I_{1} \rightarrow L_{3}$ we obtain either $L_{1} \rightleftarrows I_{1} \leftarrow L_{3}$ or $L_{3} \rightleftarrows I_{1} \leftarrow L_{1}$. From the facts that $f(0) \in \mathrm{Cl}\left(\mathbf{O} \backslash\left(B \cup C \cup I_{1}\right)\right), f^{k-1}\left(p_{e}\right) \in \mathbf{O}$, there are no basic intervals of $S^{\prime} f$-covering themselves, and by the continuity of $f$ it follows that there is $J_{1} \subset \mathbf{O}, J_{1} \in S^{\prime} \backslash\left\{I_{1}\right\}$ such that $B \leftarrow J_{1} \rightarrow I_{1}$ (notice that $J_{1}$ can be $C$ and $J_{1} \neq B$ ). Set $J=J_{1}$ if $J_{1} \neq C$ and $J=L_{2}$ if $J_{1}=C$. Clearly $x_{0} \notin J$. We have $B \leftarrow J \rightarrow I_{1}$. Moreover either $J \rightarrow B \cup A=L_{1}$ or $J \rightarrow B \cup C=L_{3}$. So we obtain either $L_{1} \leftarrow J \rightarrow I_{1}$ or $L_{3} \leftarrow J \rightarrow I_{1}$. Hence one of the following subgraphs is satisfied:

(a) $J \rightarrow I_{1} \rightleftarrows L_{i} \leftarrow J$ for $i \in\{1,3\}$;

(b) $J \rightarrow L_{j} \rightarrow I_{1} \leftarrow J$ and $I_{1} \rightleftarrows L_{i}$ for $i, j \in\{1,3\}, i \neq j$.

Clearly $I_{1} \cap L_{i}=\left\{x_{0}\right\}$ for $i \in\{1,3\}$. Then by Lemma 11.13 the result follows.

Finally assume that $f(0) \in \operatorname{Int}\left(I_{1}\right)$. Denote by $D_{b}$ and $D_{c}$ the closures of the two components of $\mathbf{O} \backslash\left\{0, f^{k-1}\left(p_{e}\right)\right\}$ such that $B \subset D_{b}$ and $C \subset D_{c}$. We have two possibilities: $f\left(x_{c}\right) \in \mathbf{I}$ or $f\left(x_{c}\right) \in \mathbf{O}$.

Suppose that $f\left(x_{c}\right) \in \mathbf{I}$. Since $f\left(x_{c}\right) \in \mathbf{I}$ and $f(0) \in \operatorname{Int}\left(I_{1}\right)$, we get that $C \rightarrow L_{1}$ and so $L_{2} \rightarrow L_{1} \leftarrow L_{3}$. From Remark $10.2 A \rightarrow\left[f(0), x_{1}\right] \subset I_{1}$ and so $L_{1} \rightarrow I_{1}$. If $I_{1} \rightarrow L_{1}$, then we have $L_{1} \rightleftarrows I_{1}$. Notice that $I_{1} \cap L_{1}=\left\{x_{0}\right\}$. Define $D_{1}=\left[p_{e}, x_{a}\right] \subset \mathbf{I}$ and $D_{2}=\mathrm{Cl}\left(\mathbf{O} \backslash\left(B \cup C \cup I_{1}\right)\right) \subset \mathbf{O}$. If $f\left(D_{1}\right) \subset D_{2}$ and $f\left(D_{2}\right) \subset D_{1}$, then by Lemma 11.14 we are done. Otherwise, there is $J \in S \cap S^{\prime}$ such that $J \rightarrow A$ and consequently either $J \rightarrow L_{1} \cup I_{1}$ or $J \rightarrow L_{2}$. Consider $L_{1} \rightleftarrows I_{1}$. If $J \rightarrow L_{1} \cup I_{1}$, from Lemma 11.5 and Proposition 7.2 the result holds. If $J \rightarrow L_{2}$, we have the paths $J \rightarrow L_{2} \rightarrow L_{1}$ and $J \rightarrow L_{2} \rightarrow I_{1}$. Then again from Lemma 11.5 and Proposition 7.2 the result follows.

Then we can suppose that $I_{1} \nrightarrow L_{1}$. Therefore $I_{1} \rightarrow L_{3}$ and $f\left(x_{1}\right) \in \mathbf{O}$. 


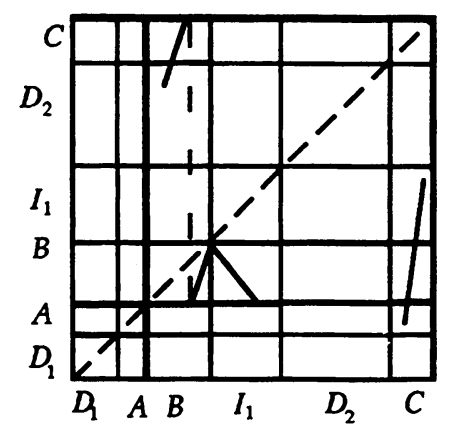

FIGURE 13.5. The map $f$ satisfies that $f\left(D_{1}\right) \subset D_{2}$ and $f\left(D_{2}\right) \subset D_{1}$.

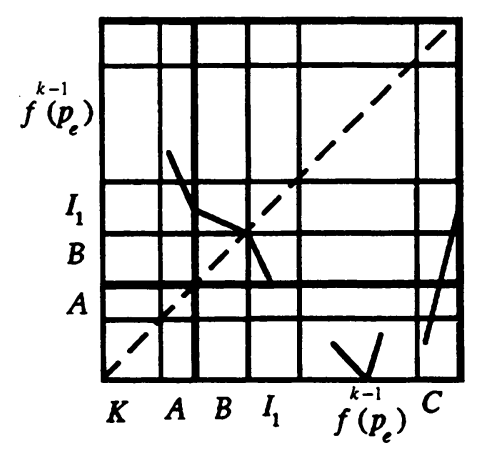

FIGURE 13.6. There are two closed subintervals $M, N \subset$ O $f$-covering $K$.

Since each basic interval of $S^{\prime}$ does not $f$-cover itself, $f(0) \in \operatorname{Int}\left(I_{1}\right)$ and there exists a closed subinterval $f$-covering $O$ (see Remark 9.6), we have that there is a closed subinterval $K \subset \mathrm{I}$ such that $K \rightarrow \mathrm{Cl}\left(\mathbf{O} \backslash\left(B \cup I_{1}\right)\right)$. From the fact that $\left\{f\left(x_{1}\right), f(0), f^{k-1}\left(p_{e}\right)\right\} \subset \mathbf{O}$ it follows that there are two closed subintervals $M, N \subset \mathbf{O} \backslash \mathrm{Cl}\left(B \cup I_{1}\right) f$-covering $K$ (see Figure 13.6). Hence we have $M \rightleftarrows K \rightleftarrows N$. Without loss of generality we can assume that $M \subset D_{b}$ and $N \subset D_{c}$. Thus we obtain a non-repetitive loop of length $n$ for each $n$ even. Since $x_{0} \notin K$ we get that $2 \mathbb{N} \subset \operatorname{Per}(f)$. If $k$ is even $S(k) \subset \operatorname{Per}(f)$ and we are done. So we can suppose that $k$ is odd. If $f\left(x_{a}\right) \in D_{b}$, then there is a minimal closed subinterval $K^{\prime} \subset\left[p_{e}, x_{a}\right]$ with endpoints elements of $P$ such that $K^{\prime} \rightleftarrows N$. Since $x_{0} \notin K^{\prime}$, from Lemma 11.15 we obtain that $n \in \operatorname{Per}(f)$ for each $n>k$ odd. Then $S(k) \subset \operatorname{Per}(f)$ and the result follows.

If $f\left(x_{a}\right) \in D_{c}$, then $A \rightarrow\left[x_{1}, f^{k-1}\left(p_{e}\right)\right] \subset D_{b}$. By the continuity of $f$ and from the fact that $\left\{f\left(x_{1}\right), f^{k-1}\left(p_{e}\right)\right\} \subset \mathbf{O}$ it follows that there is a minimal closed subinterval $M_{1} \subset\left[x_{1}, f^{k-1}\left(p_{e}\right)\right]$ (and so $x_{0} \notin M_{1}$ ) such that either $M_{1} \rightarrow L_{2}$ or $M_{1} \rightarrow L_{1} \cup I_{1}$. Therefore $A \rightarrow M_{1}$ and we obtain either the loops $M_{1} \rightleftarrows L_{2}$ and $M_{1} \rightarrow L_{2} \rightarrow L_{1} \rightarrow M_{1}$; or the loops $M_{1} \rightleftarrows L_{1}$ and $L_{1} \rightarrow I_{1} \rightarrow L_{3} \rightarrow L_{1}$. So we have a non-repetitive loop of length $n$ for each $n>k$ odd containing $M_{1}$. Since $x_{0} \notin M_{1}$ we get that $S(k) \subset \operatorname{Per}(f)$.

Finally assume that $f\left(x_{c}\right) \in \mathbf{O}$. Since $C \nrightarrow C$ we have that $L_{3} \rightarrow I_{1}$. By the continuity of $f$ and since each basic interval does not $f$-cover itseif, it 


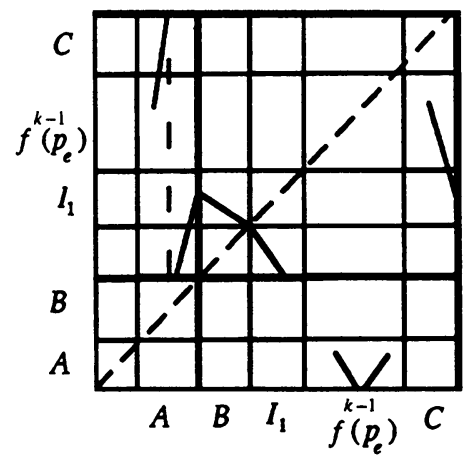

FIgURE 13.7. There is $K_{1} \subset \mathbf{I} f$-covering $\mathbf{O}$.

follows that there exists $J \in S \cap S^{\prime} \backslash\left\{I_{1}\right\}, J \subset \mathbf{O}$ such that $J \rightarrow I_{1} \cup B$. So either $J \rightarrow I_{1} \cup L_{1}$ or $J \rightarrow I_{1} \cup L_{3}$. Moreover either $I_{1} \rightarrow L_{1}$ or $I_{1} \rightarrow L_{3}$. Suppose that $L_{1} \rightarrow I_{1}$. Therefore we have either $J \rightarrow I_{1} \rightleftarrows L_{i} \leftarrow J$ for $i \in\{1,3\}$, or $J \rightarrow L_{j} \rightarrow I_{1} \leftarrow J$ and $I_{1} \rightleftarrows L_{i}$ for $i, j \in\{1,3\}, i \neq j$. By Lemma 11.13 the result holds. So from now on we can suppose that $L_{1} \nrightarrow I_{1}$. Then $A \rightarrow\left[f(0), x_{0}\right] \cup B \subset I_{1} \cup B$ and so $L_{2} \rightarrow I_{1}$. Since $A \nrightarrow A$, we get $A \rightarrow B \cup C=L_{3}$ and consequently $L_{1} \rightarrow L_{3} \leftarrow L_{2}$. If $I_{1} \rightarrow L_{3}$, then we consider the loop $I_{1} \rightleftarrows L_{3}$ and the path $I_{1} \leftarrow L_{2} \rightarrow L_{3}$. Since $0 \notin I_{1}$ and $x_{0} \notin L_{2}$, from Lemma 11.5 and Proposition 7.2 the result holds. So from now on we can suppose that $I_{1} \nrightarrow L_{3}$. Therefore $I_{1} \rightarrow L_{1}$. From the facts that $A \rightarrow B \cup C$, each basic interval does not $f$-cover itself and there is a closed subinterval $f$-covering $\mathbf{O}$, it follows that there exists a closed subinterval $K_{1} \subset I$ such that $K_{1} \rightarrow \mathbf{O}$ (see Figure 13.7). Furthermore, from the fact that $\left\{x_{0}, f\left(x_{c}\right), f^{k-1}\left(p_{e}\right)\right\} \subset \mathbf{O}$, there are two closed subintervals $M, N \subset \mathbf{O}$ such that $M \rightleftarrows K_{1} \rightleftarrows N$. Without loss of generality we can assume that $M \subset D_{b}$ and $N \subset D_{c}$. Since $x_{0} \notin K_{1}$ we get that $2 \mathbb{N} \subset \operatorname{Per}(f)$. If $k$ is even the result follows. So we can assume that $k$ is odd.

Notice that there is a closed subinterval $N^{\prime} \subset D_{c}$ such that $N^{\prime} \rightarrow \mathbf{I}$ because $\left\{f\left(x_{c}\right), f^{k-1}\left(p_{e}\right)\right\} \subset \mathbf{O}$. If $f\left(x_{a}\right) \in D_{c}$, then there is a closed subinterval $K^{\prime} \subset K_{1}$ with endpoints elements of $P \quad f$-covering $M$. Thus we have $K^{\prime} \rightleftarrows M$. By Lemma $11.15 n \in \operatorname{Per}(f)$ for each $n>k$ odd. Hence $S(k) \subset \operatorname{Per}(f)$ and we are done. So we can assume that $f\left(x_{a}\right) \in D_{b}$. Therefore $A \rightarrow D_{c}$ and consequently $L_{1} \rightarrow D_{c} \leftarrow L_{2}$. By the continuity of $f$ and since $\left\{f\left(x_{c}\right), f^{k-1}\left(p_{e}\right)\right\} \subset \mathbf{O}$, there exists a closed subinterval $N_{1} \subset D_{c}$ such that either $N_{1} \rightarrow L_{2}$ or $N_{1} \rightarrow L_{1}$ (see again Figure 13.7). Notice that $x_{0} \notin N_{1}$. If $N_{1} \rightarrow L_{1}$ then we consider the loops $L_{1} \rightleftarrows N_{1}$ and $L_{1} \rightarrow L_{3} \rightarrow I_{1} \rightarrow L_{1}$. If $N_{1} \rightarrow L_{2}$, then we consider the loops $L_{2} \rightleftarrows N_{1}$ and $L_{2} \rightarrow L_{3} \rightarrow I_{1} \rightarrow L_{1} \rightarrow N_{1} \rightarrow L_{2}$. In both cases, from Proposition 7.2 we obtain that $n \in \operatorname{Per}(f)$ for each $n>k$ odd. So $S(k) \subset \operatorname{Per}(f)$ and the proposition follows.

\section{MaPs with $f(0) \in \operatorname{Int}(A \cup B \cup C)$}

In this section we will prove Theorem 1.4(a) when $f(0) \in \operatorname{Int}(A \cup B \cup C)$. Since $f(0) \neq 0$, we have that $f(0) \in \operatorname{Int}(A \cup B \cup C)$ if and only if $f(0) \in$ $\operatorname{Int}(A) \cup \operatorname{Int}(B) \cup \operatorname{Int}(C)$. 
Lemma 14.1. Let $f$ be the standard $\sigma$ map. Suppose that $f(0) \in \operatorname{Int}(A \cup B \cup C)$ and that each basic interval of $T$ is $f$-covered by some basic interval of $T$ different from itself. If there is $J \in T$ such that $J \rightarrow J$, then Theorem 1.4(a) holds.

Proof. If $J \in S \cap S^{\prime}$, then by Proposition 11.10 we are done. So assume that $J \in\left\{L_{1}, L_{2}, L_{3}\right\}$. Since $f(0) \in \operatorname{Int}(A \cup B \cup C)$ and from the monotonicity of $f$ on each basic interval of $S^{\prime}$, it follows that $J$ does not $f$-cover basic intervals of $\left\{L_{1}, L_{2}, L_{3}\right\} \backslash\{J\}$. If $x_{0} \in J$, from the facts that $f(0) \in \operatorname{Int}(A \cup B \cup C)$ and that $f$ is monotone on each basic interval of $S^{\prime}$, it follows that $J$ does not $f$ cover basic intervals of $T$ containing $x_{0}$ different from itself. By Lemma 11.4 there is a non-repetitive loop of length $n$ for each $n \geq k+1$. By construction, there are at least two basic intervals of the above loop (perhaps they are the same) such that one does not contain 0 , and the other does not contain $x_{0}$. Then the associated sequence of $W_{i}$ 's in Proposition 7.2 is non-repetitive and so $B(k) \subset \operatorname{Per}(f)$.

Proposition 14.2. Let $f$ be the standard $\sigma$ map. Suppose that $f(0) \in$ Int $(A \cup B \cup C)$ and $x_{0} \in\left\{x_{a}, x_{b}, x_{c}\right\}$. Then Theorem 1.4(a) holds.

Proof. Denote by $I_{1}$ the basic interval with endpoint $x_{0}$ different from $A, B$ and $C$. Set $I_{1}=\left[x_{0}, x_{1}\right]$. By symmetry and from Remark 6.2 we need only consider three cases.

Case 1: $f(0) \in \operatorname{Int}(A)$ and $x_{0}=x_{a}$. Notice that from the monotonicity of $f$ on $A, A$ does not $f$-cover any basic interval. By definition of $x_{0}$ in Section 6 , the interval $\left[p_{e}, x_{0}\right)$ does not contain fixed points, so $f^{k-1}\left(p_{e}\right) \in \mathbf{O}$ and $I_{1} \rightarrow A$. Then either $I_{1} \rightarrow L_{1}$ or $I_{1} \rightarrow L_{2}$. Without loss of generality we can assume that $I_{1} \rightarrow L_{1}$. In particular $L_{1} \in T$, and $L_{1}$ is $f$-covered by some basic interval different from itself. Notice that from Remark $10.2 A$ is $f$-covered by some basic interval of $\left\{L_{1}, L_{2}, L_{3}\right\}$. So there is $z \in\left\{x_{b}, x_{c}\right\}$ such that $f(z) \in P \cap \mathbf{O}$. Hence there exists $J \in S \cap S^{\prime}, J \subset \mathbf{O}$ such that $I_{1} \leftarrow J \rightarrow A$. Therefore either $J \rightarrow L_{1}$ or $J \rightarrow L_{2}$. Notice that $\left\{x_{0}, 0\right\} \cap J=\varnothing$. Now we consider the three different possibilities for $L_{2}$, the other basic interval containing $f(0)$.

Subcase (i). Suppose that $L_{2} \notin T$. Consequently $J \rightarrow L_{1}$. Moreover $L_{3} \in T$ and $f(0) \notin L_{3}$. By Remark 11.9 each basic interval of $T$ is $f$ covered by some basic interval of $T$ different from itself. If there is a basic interval of $T f$-covering itself, by Lemma 14.1 the result follows. So suppose that there are no basic intervals of $T f$-covering themselves. Hence since $L_{2} \notin T, f\left(x_{b}\right) \in \mathbf{I}$, so we get $L_{1} \rightarrow I_{1}$. By applying Lemma 11.5 to $I_{1} \rightleftarrows L_{1}$, there is a path of length $l \leq k-1$ starting at one of the intervals $I_{1}$ or $L_{1}$ and ending at $J$. This path together with the paths $L_{1} \leftarrow J \rightarrow I_{1} \rightleftarrows L_{1}$, give us a non-repetitive loop of length $n$ for each $n \geq k+1$ in the hypotheses of Proposition 7.2, so $B(k) \subset \operatorname{Per}(f)$.

Subcase (ii). Suppose that $L_{2} \in T$ and $L_{2}$ is not $f$-covered by any basic interval of $T$ different from itself. Then $J \rightarrow L_{1}$. Moreover $C \rightarrow C$ (because $\left.L_{2} \rightarrow L_{2}\right)$ and by Lemma 11.12 taking the interval $C$, we can assume that there exists $K \in S^{\prime}, K \neq C$ such that $K \rightarrow C$, so either $K \rightarrow B$ or $K \rightarrow A$. Since $L_{2}$ only is $f$-covered by itself, $K \rightarrow B$. Hence $L_{3} \in T$. Consequently $T=S$. Hence $L_{3}$ and $L_{1}$ do not $f$-cover $L_{2}$. So $f\left(x_{b}\right) \in \mathbf{O}$ and consequently 
$L_{1} \rightarrow L_{1}$ (because $L_{1} \nrightarrow L_{2}$ ). Since $f(0) \notin L_{3}$, by Remark 11.9, $L_{3}$ is $f$ covered by some basic interval of $T$ different from itself. Notice that if $L_{2}$ $f$-covers $M$ for some $M \in T$, then $L_{3} \rightarrow M$. Set $R=T \backslash\left\{L_{2}\right\}=S \backslash\left\{L_{2}\right\}$. Therefore each basic interval of $R$ is $f$-covered by some basic interval of $R$ different from itself. Furthermore $L_{1} \rightarrow L_{1} \leftarrow J$ and $\left\{0, x_{0}\right\} \cap J=\varnothing$. So, by Lemma 11.4 interchanging $T$ by $R$, and Proposition 7.2 it follows that $B(k) \subset \operatorname{Per}(f)$.

Subcase (iii). Suppose that $L_{2} \in T$ and $L_{2}$ is $f$-covered by some basic interval different from itself. Since $f(0) \notin L_{3}$, from Remark 11.9 it follows that each basic interval of $T$ is $f$-covered by some different basic interval of $T$. Notice that $T=S$ or $T=S \backslash\left\{L_{3}\right\}$. If there is a basic interval of $T$ $f$-covering itself, then by Lemma 14.1 the result follows. Hence we can assume that each basic interval of $T$ does not $f$-cover itself. Then from the graph of $T$ it follows that $f$ has no fixed points in $\mathbf{O}$. Since $f(0) \in \mathbf{I}$, there exists a closed subinterval $K \subset\left[p_{e}, x_{0}\right]$ such that $K \rightarrow \mathbf{O}$ and $K$ has endpoints elements of $P \cup\left\{x_{0}\right\}$. From the facts that $f^{k-1}\left(p_{e}\right) \in \mathbf{O}$, and $f(0) \in A$, we get that there are two minimal closed subintervals $M, N \subset \mathbf{O}$ such that $M$ and $N$ have disjoint pairwise interiors and $M, N \rightarrow K$. Moreover $x_{0} \notin M \cup N$. By Lemma 11.6 the result follows.

Case 2: $f(0) \in \operatorname{Int}(B)$ and $x_{0}=x_{b}$. By the monotonicity of $f$ on $B$ we have that $f(B)=\left[f(0), x_{0}\right] \subset B$, otherwise $B$ contains a fixed point $y \neq x_{0}$ in contradiction with the definition of $x_{0}$ in Section 6. Notice that $B$ does not $f$-cover any basic interval. Now we will study the two basic intervals containing $f(0)$ which are $L_{1}$ and $L_{3}$.

Subcase (i). Suppose that $L_{1} \notin T$. Then by Proposition 10.8, $L_{2}, L_{3} \in T$. We claim that $L_{3}$ is $f$-covered by some $J_{1} \in T \backslash\left\{L_{3}\right\}$. Otherwise suppose that the only basic interval of $T f$-covering $L_{3}$ is itself. Therefore, since $f(B) \subset B$ and $B \cup C \rightarrow B \cup C$, we have $C \rightarrow[f(0), 0] \subset B$ and $C \rightarrow C$. So since $L_{2}=A \cup C \in T$ and $L_{2} \nrightarrow L_{3}$ it follows that $A \nrightarrow\left[f(0), x_{0}\right] \subset B$. Then either $A \rightarrow[f(0), 0] \cup A \subset B \cup A$ or $A \rightarrow[f(0), 0] \cup C \subset B \cup C$. Since $L_{1} \notin T$, we have $A \rightarrow[f(0), 0] \cup C$ and so $L_{1} \rightarrow L_{3}$. Then, since $I_{1} \cap B=\left\{x_{0}\right\}$, by the monotonicity of $f$ on each basic interval of $S^{\prime}$ we have that each basic interval of $\{A, B, C\}$ does not $f$-cover $I_{1}$. Moreover, by Remark 11.9, $I_{1}$ is $f$-covered by some different basic interval of $S^{\prime}$. So we can assume that there exists $J_{0} \in S \cap S^{\prime} \backslash\left\{I_{1}\right\}$ such that $J_{0} \rightarrow I_{1}$. Since $I_{1} \cap B=\left\{x_{0}\right\}$ and $L_{1} \notin T$, it follows that $J_{0} \rightarrow I_{1} \cup B \cup C$. In particular $J_{0} \rightarrow L_{3}$, a contradiction with the assumptions. So the claim is proved.

Since $f(0) \notin L_{2}$ and there is $J_{1} \in T \backslash\left\{L_{3}\right\}$ such that $J_{1} \rightarrow L_{3}$, by Remark 11.9 each $J \in T$ is $f$-covered by some basic interval of $T \backslash\{J\}$. By Lemma 14.1 we can suppose that for each $J \in T, J \nrightarrow J$. In particular $I_{1} \nrightarrow I_{1}$ and so $I_{1} \rightarrow B$. Hence either $I_{1} \rightarrow B \cup A=L_{1}$ or $I_{1} \rightarrow B \cup C=L_{3}$. By hypotheses $L_{1} \notin T$, so we have that $I_{1} \rightarrow L_{3}$ and thus $f\left(x_{1}\right) \in \mathbf{O}$ (by the monotonicity of $f$ on $\left.I_{1}\right)$. On the other hand $L_{3} \nrightarrow L_{3}$ and $L_{3} \nrightarrow L_{1}$, which implies that $L_{3} \rightarrow B$. So we have that $L_{3} \rightarrow I_{1}$. More concretely, $C \rightarrow\left[f(0), x_{0}\right] \subset B$ and $C \rightarrow I_{1}$. So $f\left(x_{c}\right) \in \mathbf{O}$ (otherwise $C \rightarrow A \cup C$ and consequently $L_{2} \rightarrow L_{2}$, a contradiction). Then, from the facts that $f\left(x_{1}\right) \in \mathbf{O}$, $f\left(x_{c}\right) \in \mathbf{O}, I_{1} \cap B=\left\{x_{0}\right\}$, each basic interval of $T$ does not $f$-cover itself, $L_{1} \notin T$ and by the continuity of $f$, we obtain that there exists $J \in S \cap S^{\prime} \backslash\left\{I_{1}\right\}$, 


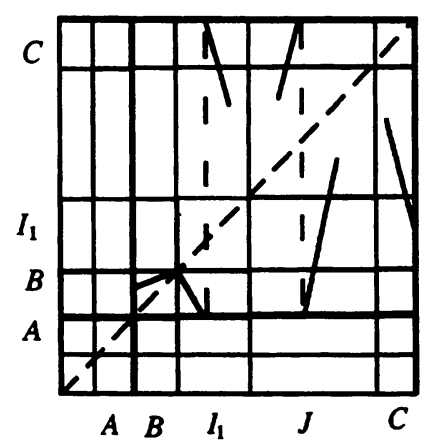

Figure 14.1. There exists $J \in S \cap S^{\prime} \backslash\left\{I_{1}\right\}, J \subset \mathbf{O}$, $f$-covering $I_{1}$ and $L_{3}$.

$J \subset \mathbf{O}$ such that $I_{1} \leftarrow J \rightarrow L_{3}$ (see Figure 14.1). Notice that $\left\{x_{0}, 0\right\} \cap J=\varnothing$. Since $I_{1} \rightleftarrows L_{3}$ and $I_{1} \cap L_{3}=\left\{x_{0}\right\}$, from Lemma 11.5 and Proposition 7.2 we obtain $B(k) \subset \operatorname{Per}(f)$.

Subcase (ii). Suppose that $L_{1} \in T$ and that $L_{1}$ is the unique interval of $T$ such that $f$-covers $L_{1}$. Then, since $f(B)=\left[f(0), x_{0}\right] \subset B$, it follows that $A \rightarrow[f(0), 0] \cup A \subset B \cup A$. Hence $f\left(x_{a}\right) \in \mathbf{I}$. Since $f(P \cap \mathbf{I}) \nsubseteq P \cap \mathbf{I}$, there exists $M \in S \cap S^{\prime}, M \subset I$ such that $M \rightarrow A$. So either $M \rightarrow A \cup B=L_{1}$ or $M \rightarrow A \cup C=L_{2}$. By the assumptions we have that $M \rightarrow L_{2}$. In particular $L_{2} \in T$ and $L_{2}$ is $f$-covered by $M \neq L_{2}$. Consequently $L_{2} \nrightarrow L_{1}$. Since $L_{2}=A \cup C, A \rightarrow[f(0), 0] \cup A \subset B \cup A$ and $B \cap I_{1}=\left\{x_{0}\right\}$ we get that $L_{2} \nrightarrow I_{1}$. Notice that each basic interval of $\{A, B, C\}$ does not $f$-cover $I_{1}$. So, by Lemma 11.12 there exists $N \in S \cap S^{\prime} \backslash\left\{I_{1}\right\}$ such that $N \rightarrow I_{1}$. Furthermore either $N \rightarrow I_{1} \cup B \cup A$ or $N \rightarrow I_{1} \cup B \cup C$. By the hypotheses we have that $N \rightarrow I_{1} \cup L_{3}$. In particular $L_{3} \in T$ and $L_{3}$ is $f$-covered by $N \neq L_{3}$. Consider $R=T \backslash\left\{L_{1}\right\}=S \backslash\left\{L_{1}\right\}$. We note that if $L_{1} f$-covers some $J \in R$, then also $L_{2} f$-covers $J$. Hence, by Remark 11.9 each basic interval of $R$ is $f$-covered by some different basic interval of $R$. Since $f\left(x_{a}\right) \in I$, by the proof of Corollary $11.11(\mathrm{~b})$, interchanging $T$ by $R$, the result holds.

Subcase (iii). Suppose that $L_{1}$ is $f$-covered by some basic interval of $T$ different from itself. Now we will study the following three possibilities for $L_{3}$.

First suppose that $L_{3} \notin T$. So by the definition of $T$ we have $L_{2} \in T$. Since $f(0) \notin L_{2}$ by Remark 11.9 each basic interval of $T$ is $f$-covered by some different basic interval of $T$. From Lemma 14.1 we can assume that each basic interval of $T$ does not $f$-cover itself. By Corollary 11.11 (b) we can suppose that $f\left(x_{a}\right) \in \mathbf{O}$. Moreover, since $I_{1} \nrightarrow I_{1}$ we have $I_{1} \rightarrow B$ and so either $I_{1} \rightarrow B \cup A=L_{1}$ or $I_{1} \rightarrow B \cup C=L_{3}$. From the assumption that $L_{3} \notin T$ we get that $I_{1} \rightarrow L_{1}$. Furthermore $A \rightarrow\left[x_{0}, f(0)\right] \subset B$ and $A \rightarrow I_{1}$ because $f(0) \in \operatorname{Int}(B), f\left(x_{a}\right) \in \mathbf{O}$ and $L_{3} \notin T$. By Remark 10.2 we get that $C \rightarrow[f(0), 0] \subset B$. Hence $L_{2}=A \cup C \rightarrow I_{1} \cup B$, and so either $L_{2} \rightarrow B \cup A=L_{1}$ or $L_{2} \rightarrow B \cup C=L_{3}$. By the assumptions, we have that $L_{2} \rightarrow L_{1}$. Therefore we have the paths $I_{1} \leftarrow L_{2} \rightarrow L_{1}$ and $I_{1} \rightleftarrows L_{1}$. Since $I_{1} \cap L_{1}=\left\{x_{0}\right\}$, by Lemma 11.5 there is a path of length $l \leq k-1$ starting at one of the intervals $I_{1}$ or $L_{1}$ and ending at $L_{2}$. If the path starts at $L_{1}$, then we consider the loops $L_{1} \rightarrow \cdots \rightarrow L_{2} \rightarrow L_{1} \rightarrow I_{1} \rightarrow L_{1}, L_{1} \rightarrow \cdots \rightarrow L_{2} \rightarrow I_{1} \rightarrow L_{1}$ and $L_{1} \rightleftarrows I_{1}$ of 
lengths $l+3 \leq k+2, l+2 \leq k+1$ and 2 respectively. If the path starts at $I_{1}$, then we consider the loops $I_{1} \rightarrow \cdots \rightarrow L_{2} \rightarrow I_{1}, I_{1} \rightarrow \cdots \rightarrow L_{2} \rightarrow L_{1} \rightarrow I_{1}$ and $I_{1} \rightleftarrows L_{1}$ of lengths $l+1 \leq k, l+2 \leq k+1$ and 2 respectively. Clearly $0 \notin I_{1}$ and $x_{0} \notin L_{2}$. Notice that all the above loops contain $I_{1}$ and $L_{2}$. Then by the construction of the loops and by Proposition 7.2 the result holds.

Now suppose that $L_{3} \in T$ and $L_{3}$ is the unique basic interval of $T f$ covering $L_{3}$. Then, since $B \rightarrow\left[f(0), x_{0}\right] \subset B$, we have that $C \rightarrow[f(0), 0] \subset$ $B$ and $C \rightarrow C$. By Lemma 11.12 we can assume that $C$ is $f$-covered by some $M \in S^{\prime}, M \neq C$. Moreover, by the hypotheses we have that $L_{1} \in T$ and so $L_{1} \nrightarrow L_{3}$. We claim that $M \neq A$. Otherwise, suppose $M=A$. Therefore, since $L_{1} \nrightarrow L_{3}$ it follows that $A \rightarrow \mathbf{O} \backslash(f(0), 0)$ and $A \rightarrow A$. In particular $L_{2} \rightarrow L_{2}$ and $L_{2} \rightarrow L_{3}$, in contradiction with the assumptions. So the claim is proved. Notice that $M \neq B$, because $f(B) \subset B$. Then we have $M \notin\{A, B, C\}$, and so $M \in S \cap S^{\prime}$. Since $M \rightarrow C$ and $M \rightarrow C \cup B=L_{3}$, we get that $M \rightarrow C \cup A=L_{2}$. In particular $L_{2} \in T$ and $L_{2}$ is $f$-covered by $M \neq L_{2}$. Notice that if $L_{3} \rightarrow N$, for some $N \in T \backslash\left\{L_{3}\right\}$, then $C \rightarrow N$ and consequently $L_{2} \rightarrow N$. Set $R=T \backslash\left\{L_{3}\right\}=S \backslash\left\{L_{3}\right\}$. Therefore each basic interval of $R$ is $f$-covered by some different basic interval of $R$. From the facts that $L_{1} \nrightarrow L_{3}, L_{2} \nrightarrow L_{3}, C \rightarrow[0, f(0)] \cup C \subset B \cup C$ and $B \rightarrow\left[f(0), x_{0}\right] \subset B$, it follows that $L_{1} \rightarrow L_{1}$. Then from the proof of Lemma 14.1, interchanging $T$ by $R$ the result holds.

Finally suppose that $L_{3} \in T$ and $L_{3}$ is $f$-covered by some different basic interval of $T$. Since $f(0) \notin L_{2}$, by Remark 11.9 we have that each $J \in T$ is $f$-covered by some interval of $T \backslash\{J\}$. From Lemma 14.1 we can assume that for each $J \in T, J \nrightarrow J$. In particular $I_{1} \nrightarrow I_{1}$ and so either $I_{1} \rightarrow L_{1}$ or $I_{1} \rightarrow L_{3}$. We claim that $C \nrightarrow C$. Otherwise suppose that $C \rightarrow C$. Therefore either $C \rightarrow[0, f(0)] \subset B$ and so $L_{3} \rightarrow L_{3}$; or $C \rightarrow C \cup A$ and $L_{2} \rightarrow L_{2}$ in contradiction with the fact that for each $J \in T, J \nrightarrow J$. Thus the claim is proved. From Corollary 11.11(a) we can assume that $f^{k-1}\left(p_{e}\right) \in \mathbf{O}$. By Remark 9.6 there is a closed subinterval $K \subset \sigma$ such that $K \rightarrow \mathbf{O}$. From the fact that $f(B)=\left[f(0), x_{0}\right] \subset B, J \nrightarrow J$ for each $J \in T$ and $C \nrightarrow C$, it follows that $K \nsubseteq \mathbf{O}$. Then there exists $K^{\prime} \subset \mathbf{I}$ such that $K^{\prime} \rightarrow \mathbf{O} \backslash \operatorname{Int}(B)$ (see Figure 14.2). From the fact that $\left\{f^{k-1}\left(p_{e}\right), x_{0}, f(0)\right\} \subset \mathbf{O}$ it follows that there are two minimal closed subintervals $M, N \subset \mathbf{O} \backslash \operatorname{Int}(B) \quad f$-covering $K^{\prime}$. Then

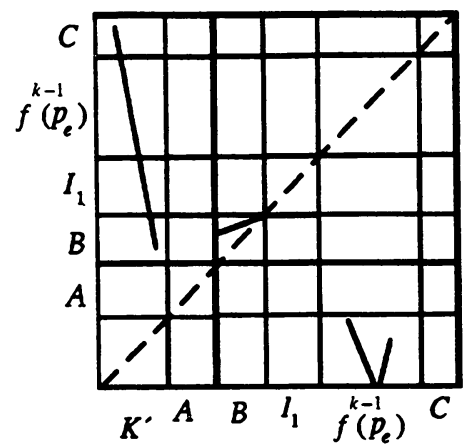

FIGURE 14.2. There is $K^{\prime} \subset K \cap I$ such that $K^{\prime} \rightarrow$ $\mathbf{O} \backslash \operatorname{Int}(B)$. 


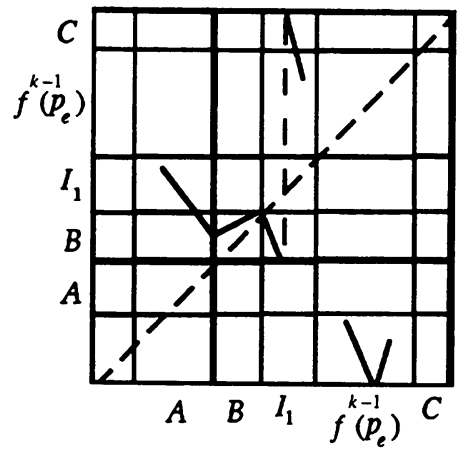

Figure 14.3. There is a closed subinterval $M \subset \mathbf{O}$ $f$-covering $L_{2}$ or $L_{1} \cup I_{1}$.

we get $M \rightleftarrows K^{\prime} \rightleftarrows N$. Since $x_{0} \notin K^{\prime}$ we obtain $2 \mathbb{N} \subset \operatorname{Per}(f)$. If $k$ is even, then $S(k) \subset \operatorname{Per}(f)$ and we are done. Hence we can assume that $k$ is odd. Denote by $D_{c}$ and $D_{b}$ the closures of the two components of $O \backslash\left\{0, f^{k-1}\left(p_{e}\right)\right\}$ such that $B \subset D_{b}$ and $C \subset D_{c}$.

First assume that $A \rightarrow I_{1}$. Then $L_{1} \rightarrow I_{1} \leftarrow L_{2}$. From Remark 10.2 and since $C \nrightarrow C$, we have that $C \rightarrow[f(0), 0] \cup A \subset B \cup A$. Consequently $L_{2} \rightarrow L_{1} \leftarrow L_{3}$. On the other hand, either $I_{1} \rightarrow L_{1}$ or $I_{1} \rightarrow L_{3}$. Suppose that $I_{1} \rightarrow L_{1}$. If $f(P \cap \mathbf{I})=P \cap \mathbf{O}$ and $f(P \cap \mathbf{O})=P \cap \mathbf{I}$, then $k$ must be even, in contradiction with the assumptions. Therefore there is $J \in S \cap S^{\prime}$ $f$-covering either $L_{1} \cup I_{1}$ or $L_{2}$. So we consider the loop $I_{1} \rightleftarrows L_{1}$ and the path either $L_{1} \leftarrow J \rightarrow I_{1}$ or $L_{1} \leftarrow L_{2} \leftarrow J \rightarrow L_{2} \rightarrow I_{1}$. From Lemma 11.5 we obtain a non-repetitive loop of length $n$ for each $n \geq k+1$ containing $J$. Since $\left\{x_{0}, 0\right\} \cap J=\varnothing$ from Proposition 7.2 the result follows. Therefore we can assume that $I_{1} \nrightarrow L_{1}$. Hence $I_{1} \rightarrow L_{3}$ and by the monotonicity, $f\left(x_{1}\right) \in \mathbf{O}$. Since $\left\{f^{k-1}\left(p_{e}\right), f(0)\right\} \subset \mathbf{O}$ it follows that there exists a closed subinterval $N \subset D_{c} f$-covering I. Moreover, since $\left\{f\left(x_{1}\right), f^{k-1}\left(p_{e}\right)\right\} \subset \mathbf{O}$, by the continuity it follows that there exists a closed subinterval $M \subset D_{b}$ such that $x_{0} \notin M$ and either $M \rightarrow L_{2}$ or $M \rightarrow L_{1} \cup I_{1}$ (see Figure 14.3). If $f\left(x_{a}\right) \in D_{b}$, then there exists a closed subinterval $K^{\prime} \subset\left[p_{e}, x_{a}\right] \subset \mathbf{I}$ with endpoints elements of $P$ such that $K^{\prime} \rightarrow D_{c}$. Therefore we have $K^{\prime} \rightleftarrows N$ and $x_{0} \notin K^{\prime}$. From Lemma 11.15 the result holds. So suppose that $f\left(x_{a}\right) \in D_{c}$. Then $A \rightarrow D_{b}$. In particular $L_{1} \rightarrow M \leftarrow L_{2}$. We consider the loops either $M \rightleftarrows L_{1} \rightarrow I_{1} \rightarrow L_{3} \rightarrow L_{1}$; or $M \rightleftarrows L_{2} \rightarrow L_{1} \rightarrow M$. Thus we obtain a nonrepetitive loop of length $n$ for each $n>k$ odd containing $M$. Since $x_{0} \notin M$ we have $S(k) \subset \operatorname{Per}(f)$.

Finally assume that $A \nrightarrow I_{1}$. Since $J \nrightarrow J$ for each $J \in T$, it follows that $A \rightarrow[f(0), 0] \cup C \subset B \cup C$ and so $L_{1} \rightarrow L_{3}$. In particular $f\left(x_{a}\right) \in \mathbf{O}$. Notice that the closed subinterval $K f$-covering $\mathbf{O}$ is contained in I. If $f\left(x_{a}\right) \in D_{c}$, then there is $K^{\prime} \subset I, K^{\prime}$ with endpoints elements of $P$ such that $K^{\prime} \rightarrow D_{b}$. From the fact that $\left\{x_{0}, f^{k-1}\left(p_{e}\right)\right\} \subset \mathbf{O}$, there is a closed subinterval $M \subset D_{c}$ such that $M \rightarrow K^{\prime}$. Then we have $K^{\prime} \rightleftarrows M$ and $x_{0} \notin K^{\prime}$. By Lemma 11.15 the result holds. Otherwise $f\left(x_{a}\right) \in D_{b}$. Hence $A \rightarrow D_{c}$ and consequently $L_{1} \rightarrow D_{c} \leftarrow L_{2}$. Now we have two possibilities: $f\left(x_{c}\right) \in \mathbf{O}$ or $f\left(x_{c}\right) \in \mathbf{I}$.

First suppose that $f\left(x_{c}\right) \in \mathbf{O}$. Then we get that $C \rightarrow I_{1}$ (because $L_{3} \nrightarrow L_{3}$ ) 


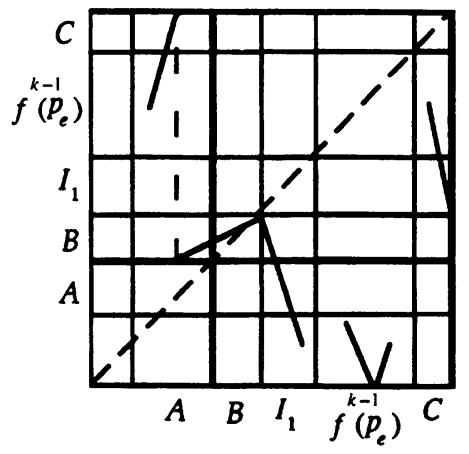

FIGURE 14.4. There is a basic interval of $D_{c} f$-covering $L_{1}$ or $L_{2}$.

and so $L_{3} \rightarrow I_{1} \leftarrow L_{2} \rightarrow L_{3}$. On the other hand either $I_{1} \rightarrow L_{3}$ or $I_{1} \rightarrow L_{1}$. If $I_{1} \rightarrow L_{3}$, then $f\left(x_{1}\right) \in \mathbf{O}$. Hence there is a basic interval $M \in S \cap S^{\prime}, M \subset \mathbf{O}$ such that either $M \rightarrow L_{3} \cup I_{1}$ or $M \rightarrow L_{2}$. Therefore we consider the loop $L_{3} \rightleftarrows I_{1}$ and either the paths $L_{3} \leftarrow M \rightarrow I_{1}$; or $L_{3} \leftarrow L_{2} \leftarrow M \rightarrow L_{2} \rightarrow I_{1}$. So from Lemma 11.5 and Proposition 7.2 the result holds. Otherwise, $I_{1} \rightarrow L_{1}$. Since $\left\{f\left(x_{c}\right), f^{k-1}\left(p_{e}\right)\right\} \subset \mathbf{O}$, there exists $M \in S \cap S^{\prime}, M \subset D_{c}$, such that either $M \rightarrow L_{1}$ or $M \rightarrow L_{2}$ (see Figure 14.4). From the fact that $L_{1} \rightarrow D_{c} \leftarrow$ $L_{2}$ we obtain either $M \rightleftarrows L_{1}$ or $L_{1} \rightarrow M \rightleftarrows L_{2}$. So we consider either the loops $L_{1} \rightarrow L_{3} \rightarrow I_{1} \rightarrow L_{1}$ and $L_{1} \rightleftarrows M$; or the loops $L_{2} \rightarrow L_{3} \rightarrow I_{1} \rightarrow L_{1} \rightarrow$ $M \rightarrow L_{2}$ and $L_{2} \rightleftarrows M$. So we obtain a non-repetitive loop of length $n$ for each $n \geq 5$ odd containing $M$. Since $x_{0} \notin M$ the result follows.

Finally suppose that $f\left(x_{c}\right) \in I$. Let $p_{1} \in I_{1}$ be such that $f\left(p_{1}\right)=f(0)$. Set $U=\left[0, p_{1}\right] \subset B \cup I_{1}$. We define the map $g: \sigma \longrightarrow \sigma$ as $\left.g\right|_{\sigma \backslash U}=\left.f\right|_{\sigma \backslash U}$ and $\left.g\right|_{U}=\{f(0)\}$. Clearly $g(0)=f(0) \in \mathbf{O}$ and $g$ has a fixed point $z \in B$ (see Figure 14.5). So $g$ is a $\sigma$ map. Moreover $P$ is a periodic orbit of period $k$ for $g$. Of course $\operatorname{Per}(g) \subset \operatorname{Per}(f)$. We remark that $g$ is monotone on each basic interval associated to $P \cup\{z\} \cup\{0\}$. If we denote by $A^{\prime}, B^{\prime}$ and $C^{\prime}$ the three basic intervals associated to $P \cup\{z\} \cup\{0\}$ with 0 as endpoint, then we have $B^{\prime}=[0, z]$ and $g(0)=g(z)$. From Remark 12.6 the result follows.

Case 3: $f(0) \in \operatorname{Int}(B)$ and $x_{0}=x_{c}$. We will deal with two basic intervals containing $f(0)$ which are $L_{1}$ and $L_{3}$. Notice that by definition of $x_{0}$ in Section $6, B$ does not contain fixed points of $f$. So $B \rightarrow\left[f(0), x_{b}\right] \subset B$.

Subcase (i). Suppose that $L_{3} \notin T$. So by definition of $T$ we have that $L_{1}, L_{2} \in T$. Notice that $C$ does not $f$-cover $C$ (otherwise $L_{3}=B \cup C \rightarrow$ $B \cup C)$. Therefore $C \rightarrow\left[f(0), x_{b}\right] \subset B$ and $C \rightarrow I_{1}$. So $L_{2} \rightarrow I_{1}$. Since $f(0) \in \operatorname{Int}(B)$ and $B \rightarrow\left[f(0), x_{b}\right] \leftarrow C$, by Remark 10.2 we get that $A \rightarrow$ $[f(0), 0] \subset B$. Furthermore, from the fact that $L_{3} \notin T$ we obtain that $f\left(x_{a}\right) \in$ I (otherwise $L_{1}$ and $L_{2} f$-cover $L_{3}$ ) and $A \rightarrow A$. Thus $L_{1} \rightarrow L_{1} \leftarrow L_{2}$. Therefore $L_{1}$ is $f$-covered by a basic interval of $T$ different from itself. Since $f(0) \notin L_{2}$, from Remark 11.9 we have that each basic interval of $T$ is $f$ covered by some basic interval different from itself. Since $L_{1} \rightarrow L_{1}$ by Lemma 14.1 the result holds.

Subcase (ii). Suppose that $L_{3} \in T$ and there are no basic intervals of $T$ different from $L_{3} f$-covering $L_{3}$. Since $B \rightarrow\left[f(0), x_{b}\right] \subset B$ and $L_{3} \rightarrow L_{3}$ it follows that $C \rightarrow[0, f(0)] \cup C \subset B \cup C$. 


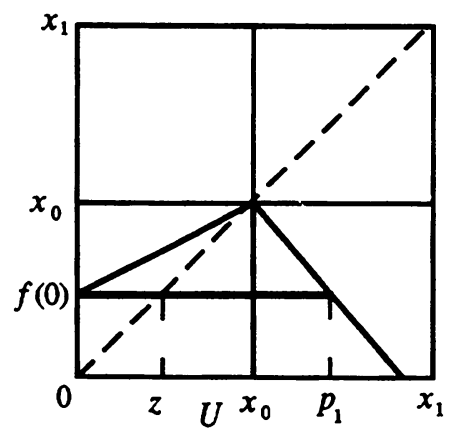

FIGURE 14.5. The new $\sigma$ map $g$ in $U$.

First assume that $L_{1} \notin T$. Then by the definition of $T$ we have that $L_{2} \in T$. Consequently $L_{2} \nrightarrow L_{3}$ and so $A \nrightarrow\left[f(0), x_{b}\right] \subset B$. Therefore $A \rightarrow[0, f(0)] \subset B$. Moreover either $A \rightarrow A$ and $L_{1} \rightarrow L_{1}$, or $A \rightarrow C$ and $L_{1} \rightarrow L_{3}$. By the assumption $L_{1} \notin T$ we get $A \rightarrow C$ and $L_{1} \rightarrow L_{3}$. Thus $f\left(x_{a}\right) \in \mathbf{O}$ and $A \rightarrow I_{1}$. In particular $L_{2} \rightarrow I_{1}$. Notice that each basic interval $J_{0} \in S \cap S^{\prime}$ does not $f$-cover $B$; otherwise either $J_{0} \rightarrow B \cup A=L_{1}$ or $J_{0} \rightarrow B \cup C=L_{3}$ in contradiction with the assumptions. Set $R=T \backslash\left\{L_{3}\right\}=$ $S \backslash\left\{L_{1}, L_{3}\right\}$. Notice that $L_{1} \cap L_{3}=B$. We claim that if $L_{3} f$-covers $M$ for some $M \in R$, then there is $J \in R, J \neq M$ such that $J \rightarrow M$. Now we prove the claim. Since $f(C) \subset L_{3}, B \rightarrow M$. Suppose that $M \subset \mathbf{O}$. So from the facts that $J \nrightarrow B$ for each $J \in S \cap S^{\prime}, x_{b} \in P$ and by the continuity of $f$, it follows that there is $J \in R, J \neq M$ such that $J \rightarrow M$ (see Figure 14.6). Suppose that $M \subset \mathbf{I}$. Then $f\left(x_{b}\right) \in \mathbf{I}$. Hence from the facts that $f\left(x_{b}\right) \in \mathbf{I}, f\left(x_{c}\right) \in \mathbf{O}$ and by the continuity of $f$ it follows that there is $J \in R, J \subset \mathbf{O}$ (and so $J \neq M$ ) such that $J \rightarrow M$. Finally suppose that $M=L_{2}$. Since $A \rightarrow C$ and $C \rightarrow C$, clearly $L_{2} \nrightarrow L_{2}$. Since $f\left(x_{a}\right) \in \mathbf{O}, f\left(x_{c}\right) \in \mathbf{O}, f\left(f^{k-1}\left(p_{e}\right)\right) \in \mathbf{I}$, each basic interval of $S \cap S^{\prime}$ does not $f$-cover $B$ and by the continuity of $f$, it follows that there is $J \in S \cap S^{\prime}$ (and so $J \neq L_{2}$ ) such that $J \rightarrow L_{2}$ (see again Figure 14.6). So the claim is proved.

Hence from Remark 11.9 each basic interval of $R$ is $f$-covered by some different basic interval of $R$. If there is $J \in R$ such that $J \rightarrow J$, from the

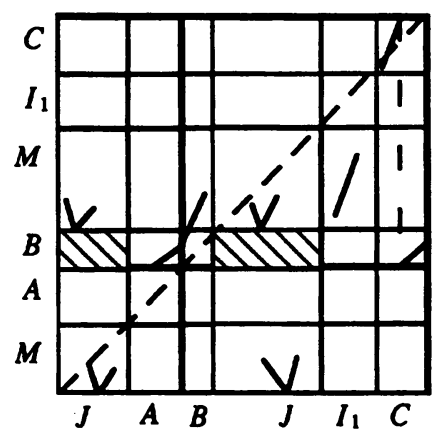

Figure 14.6. There is $J \in T, J \neq M$ such that $J \rightarrow$ $M$. 


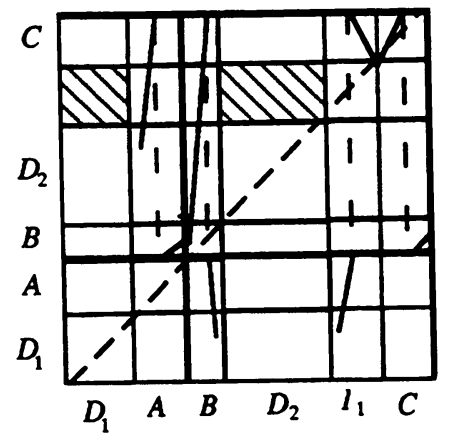

FIgURE 14.7. The graph of $f$ with $f\left(D_{1}\right) \subset D_{2}$ and $f\left(D_{2}\right) \subset D_{1}$.

proof of Lemma 14.1, replacing $T$ by $R$, the result follows. So we can assume that for each $J \in R \quad J \nrightarrow J$. By the proof of Corollary 11.11(a) we can suppose that $f^{k-1}\left(p_{e}\right) \in \mathbf{O}$. Moreover $I_{1} \nrightarrow I_{1}$ and so $I_{1} \rightarrow L_{2}$ (since $\left.I_{1} \nrightarrow L_{3}\right)$. We have the loop $I_{1} \rightleftarrows L_{2}$, with $I_{1} \cap L_{2}=\left\{x_{0}\right\}$. If there is $J \in S \cap S^{\prime}$ such that $I_{1} \leftarrow J \rightarrow L_{2}$, then by the proof of Lemma 11.5 and Proposition 7.2 (replacing $T$ by $R$ ) the result holds. Consequently we can assume that for each $J \in S \cap S^{\prime} \backslash\left\{I_{1}\right\}, J \nrightarrow I_{1}$ and $J \nrightarrow L_{2}$. Set $D_{1}=\left[p_{e}, x_{a}\right] \subset I$ and $D_{2}=\mathrm{Cl}\left(\mathbf{O} \backslash\left(B \cup C \cup I_{1}\right)\right)$ (see Figure 14.7). Therefore we get that $f\left(D_{1}\right) \subset D_{2}$ and $f\left(D_{2}\right) \subset D_{1}$. Then by Lemma 11.14 the result holds.

Finally assume that $L_{1} \in T$. Thus we have $L_{1} \nrightarrow L_{3}$. Suppose that $L_{1}$ does not $f$-cover $L_{1}$. Then since $L_{1} \nrightarrow L_{3}$, we get that $A \rightarrow\left[f(0), x_{b}\right]$. Consequently $L_{2} \rightarrow L_{3}$ and so $L_{2} \notin T$. Since there are no basic intervals of $T f$-covering $L_{2}, L_{3}$ is the unique interval of $T f$-covering $L_{3}$, and $C=L_{2} \cap L_{3}$, it follows that $C$ is not $f$-covered by any basic interval of $S^{\prime}$ different from itself. Hence by Lemma 11.12 the result follows. So in the rest of the proof of this subcase we can assume that $L_{1} \rightarrow L_{1}$. Then $f\left(x_{a}\right) \in \mathbf{I}$. Suppose that $L_{1}$ is $f$-covered by some different basic interval of $T$. Notice that $L_{3} \nrightarrow L_{1}$. Set $R=T \backslash\left\{L_{3}\right\}$. We remark that if $L_{3} \rightarrow M$, for $M \in R$, then $B \rightarrow M$ (because $f\left(x_{0}\right)=x_{0}$ and $\left.f(C) \subset L_{3}\right)$. Consequently $L_{1} \rightarrow M$. So by Remark 11.9 each $J \in T$ is $f$-covered by some interval $T \backslash\{J\}$. Since $L_{1} \rightarrow L_{1}$, by the proof of Lemma 14.1, replacing $T$ by $R$ the result holds.

Now we can suppose that $L_{1} \rightarrow L_{1}$ and $L_{1}$ is not $f$-covered by any basic interval of $T \backslash\left\{L_{1}\right\}$. Then $A \rightarrow[f(0), 0] \cup A \subset B \cup A$. Since $f(P \cap \mathbf{I}) \nsubseteq P \cap \mathbf{I}$, there exists $z \in P \cap I$ such that $f(z) \in P \cap \mathbf{O}$. Then, since $x_{a}, z \in \mathbf{I}, f\left(x_{a}\right) \in \mathbf{I}$ and $f(z) \in \mathbf{O}$, by the continuity of $f$ it follows that there exists $J_{0} \in S \cap S^{\prime}$, $J_{0} \subset I$ such that $J_{0} \rightarrow A$. So either $J_{0} \rightarrow A \cup B=L_{1}$ or $J_{0} \rightarrow A \cup C=L_{2}$. By the hypotheses we get that $J_{0} \rightarrow L_{2}$. In particular $L_{2} \in T$. Notice that each basic interval $J \in S \cap S^{\prime}$ does not $f$-cover $B$, because otherwise either $J \rightarrow B \cup A=L_{1}$ or $J \rightarrow B \cup C=L_{3}$ in contradiction with the assumptions. Set $R=T \backslash\left\{L_{1}, L_{3}\right\}=S \backslash\left\{L_{1}, L_{3}\right\}$. We have $L_{1} \cap L_{3}=B$ and $f(0) \in \operatorname{Int}(B)$. We claim that if $L_{1}$ or $L_{3} f$-covers $M$ for some $M \in R$, then there is $J \in R$, $J \neq M$ such that $J \rightarrow M$. Now we prove the claim. If $M=L_{2}$ then take $J=J_{0}$ and we are done. Suppose that $M \subset \mathbf{O}$. Hence from the facts that $J \nrightarrow B$ for each $J \in S \cap S^{\prime}, x_{b} \in P$, and by the continuity of $f$ it follows (like in Figure 14.6) that there is $J \in R, J \neq M$ such that $J \rightarrow M$. Suppose that 


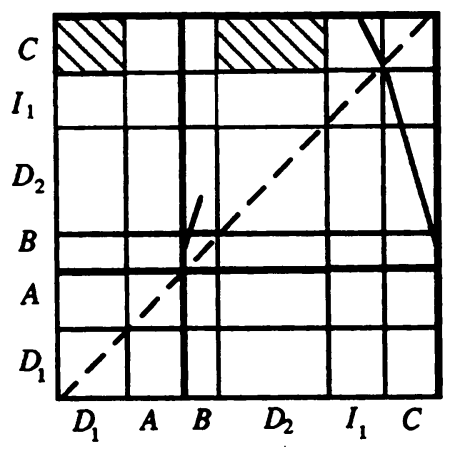

Figure 14.8. For each $J \in S \cap S^{\prime}, J \nrightarrow C$.

$M \subset$ I. If $A \rightarrow M$, then take $J=L_{2}$ and we are done; otherwise, we have $B \rightarrow M$ (because $f(C) \subset \mathbf{O})$. Therefore $f\left(x_{b}\right) \in \mathbf{I}$. Since $M \subset\left[f\left(x_{b}\right), 0\right]$, $f\left(x_{c}\right) \in \mathbf{O}$ and by the continuity of $f$, there exists $J \in S \cap S^{\prime}, J \subset \mathbf{O}$ $f$-covering $M$. Notice that $J \neq M$. So the claim is proved. Hence each basic interval of $R$ is $f$-covered by some different basic interval of $R$. Since $f\left(x_{a}\right) \in \mathbf{I}$, from the proof of Corollary 11.11(b) interchanging $T$ by $R$ the result holds.

Subcase (iii). Suppose that $L_{3} \in T$ and $L_{3}$ is $f$-covered by some basic interval of $T$ different from itself. Now we shall deal with $L_{1}$.

First we assume that $L_{1} \notin T$, then by the definition of $T$ we have that $L_{2} \in T$. Since $f(0) \notin L_{2}$, by Remark 11.9 we have that each basic interval of $T$ is $f$-covered by some different basic interval of $T$. From Lemma 14.1 we can assume that each basic interval of $T$ does not $f$-cover itself. By Corollary 11.11 we can suppose that $f^{k-1}\left(p_{e}\right) \in \mathbf{O}$ and $f\left(x_{a}\right) \in \mathbf{O}$. Since $x_{c}=x_{0}$, $f(0) \in B$ and $L_{3} \rightarrow L_{3}$ we have that $C \rightarrow I_{1}$. Then $L_{2} \rightarrow I_{1}$ and $L_{3} \rightarrow I_{1}$. Furthermore, since $I_{1} \nrightarrow I_{1}$, we get $I_{1} \rightarrow C$. Moreover either $I_{1} \rightarrow C \cup A=L_{2}$ or $I_{1} \rightarrow C \cup B=L_{3}$. Suppose that there is $J \in S \cap S^{\prime} \backslash\left\{I_{1}\right\}$ such that $J \rightarrow C$. Then, since $f\left(x_{0}\right)=x_{0}, J \rightarrow I_{1}$. Moreover either $J \rightarrow C \cup A=L_{2}$ or $J \rightarrow C \cup B=L_{3}$. Thus one of the following subgraphs is satisfied:

(a) $J \rightarrow I_{1} \rightleftarrows L_{i} \leftarrow J$ for some $i \in\{2,3\}$;

(b) $J \rightarrow L_{j} \rightarrow I_{1} \leftarrow J$ and $I_{1} \rightleftarrows L_{i}$ for $i, j \in\{2,3\}, i \neq j$.

By Lemma 11.13 the result follows. So we can assume that for each $J \in$ $S \cap S^{\prime} \backslash\left\{I_{1}\right\}, J \nrightarrow C$ (see Figure 14.8). Set $D_{1}=\left[p_{e}, x_{a}\right] \subset I$ and $D_{2}=$ $\mathrm{Cl}\left(\mathrm{O} \backslash\left(B \cup C \cup I_{1}\right)\right)$. Therefore we get that $f\left(D_{1}\right) \subset D_{2}$ and $f\left(D_{2}\right) \subset D_{1}$ (because $L_{1} \notin T$ ). By Lemma 11.14 the result holds.

Assume that $L_{1} \in T$ and $L_{1}$ is the unique interval of $T f$-covering $L_{1}$. Since $B \rightarrow\left[f(0), x_{b}\right] \subset B$ and $L_{1} \rightarrow L_{1}$, we have that $A \rightarrow[f(0), 0] \subset B$ and $A \rightarrow A$. So $f\left(x_{a}\right) \in \mathbf{I}$. Clearly $f(P \cap I) \nsubseteq P \cap I$. Hence there is $z \in P \cap I$ such that $f(z) \in P \cap \mathbf{O}$. Moreover, since $f\left(x_{a}\right) \in \mathbf{I}$ and by the continuity of $f$ it follows that there exists $J \in S \cap S^{\prime}, J \subset I$ such that $J \rightarrow A$. Therefore either $J \rightarrow A \cup B=L_{1}$ or $J \rightarrow A \cup C \cup I_{1}$. By the assumptions we get that $J \rightarrow L_{2} \cup I_{1}$. In particular $L_{2} \in T$. Set $R=T \backslash\left\{L_{1}\right\}=S \backslash\left\{L_{1}\right\}$. Notice that $L_{1} \nrightarrow L_{3}$. Furthermore if $L_{1} \rightarrow M$ for some $M \in R$, then $A \rightarrow M$ or $B \rightarrow M$. Consequently $L_{2} \rightarrow M$ or $L_{3} \rightarrow M$. Hence each basic interval of $R$ is $f$-covered by some different basic interval of $R$ (see Remark 11.9). Since 
$f\left(x_{a}\right) \in \mathbf{I}$, by the proof of Corollary $11.11(\mathrm{~b})$, replacing $T$ by $R$, the result follows.

Finally assume that $L_{1} \in T$ and that $L_{1}$ is $f$-covered by some different basic interval of $T$. Since $f(0) \notin L_{2}$, from Remark 11.9 each $J \in T$ is $f$-covered by some basic interval of $T \backslash\{J\}$. Then by Lemma 14.1 we can suppose that each basic interval of $T$ does not $f$-cover itself. So we have that $I_{1} \nrightarrow I_{1}$ and $L_{3} \nrightarrow L_{3}$. Therefore $I_{1} \rightarrow C$ and $C \rightarrow I_{1}$ (because $\left.f\left(x_{0}\right)=x_{0}\right)$. Thus $L_{2}$ and $L_{3} f$-cover $I_{1}$. Moreover either $I_{1} \rightarrow L_{2}$ or $I_{1} \rightarrow L_{3}$. Suppose that there is $J \in S \cap S^{\prime} \backslash\left\{I_{1}\right\}$ such that $J \rightarrow C$. Then either $J \rightarrow L_{2} \cup I_{1}$ or $J \rightarrow L_{3} \cup I_{1}$. Hence one of the following subgraphs is satisfied:

(a) $J \rightarrow I_{1} \rightleftarrows L_{i} \leftarrow J$ for some $i \in\{2,3\}$;

(b) $J \rightarrow L_{j} \rightarrow I_{1} \leftarrow J$ and $I_{1} \rightleftarrows L_{i}$ for $i, j \in\{2,3\}, i \neq j$.

By Lemma 11.13 the result holds. Consequently we can assume that for each $J \rightarrow S \cap S^{\prime} \backslash\left\{I_{1}\right\}, J \nrightarrow L_{2}$ and $J \nrightarrow L_{3}$. Set $D_{1}=\left[p_{e}, x_{a}\right] \subset I$ and $D_{2}=$ $\mathbf{O} \backslash \operatorname{Int}\left(B \cup C \cup I_{1}\right)$. Therefore we get that $f\left(D_{1}\right) \subset D_{2}$ and $f\left(D_{2}\right) \subset D_{1}$. By Lemma 11.14 the result holds.

Proposition 14.3. Let $f$ be the standard $\sigma$ map. Suppose that $f(0) \in$ $\operatorname{Int}(A \cup B \cup C)$ and $x_{0} \notin\left\{x_{a}, x_{b}, x_{c}\right\}$. Then Theorem 1.4(a) holds.

Proof. We denote by $I_{1}, I_{2}$ the two basic intervals with $x_{0}$ as endpoint. These basic intervals exist in virtue of Remark 6.4. Since $x_{0} \notin\left\{x_{a}, x_{b}, x_{c}\right\}$, we have that $I_{1}, I_{2} \in S \cap S^{\prime}$. By symmetry and Remark 6.2 we only need consider the two following cases.

Case 1: $f(0) \in \operatorname{Int}(A)$ and $x_{0} \in \mathbf{I}$. Without loss of generality we can assume that $I_{1} \subset\left[p_{e}, x_{0}\right]$. So by the definition of $x_{0}$ in Section 6 we have that $\left[p_{e}, x_{0}\right)$ does not contain fixed points of $f$. Consequently $I_{1} \rightarrow I_{1}$. So $I_{1} \rightarrow I_{2}$. Since $f(P \cap I) \nsubseteq P \cap I$, it follows that there is $z \in P \cap I$ such that $f(z) \in P \cap \mathbf{O}$. By hypotheses $x_{0} \in \mathbf{I}$. Hence, by the continuity of $f$, there exists $J_{0} \in S \cap S^{\prime}$ such that $J_{0} \rightarrow A$. Therefore either $J_{0} \rightarrow A \cup B=L_{1}$ or $J_{0} \rightarrow A \cup C=L_{2}$. Without loss of generality we can assume that $J_{0} \rightarrow A \cup B=L_{1}$. Thus $L_{1} \in T$ and $L_{1}$ is $f$-covered by $J_{0} \in S \cap S^{\prime}, J_{0} \neq L_{1}$. Since $\left[p_{e}, x_{0}\right)$ does not contain fixed points of $f$ we get that $f^{k-1}\left(p_{e}\right) \notin\left[p_{e}, x_{0}\right]$. So since $I_{1} \cap I_{2}=\left\{x_{0}\right\}$, $f(0) \in A$ and $f\left(f^{k-1}\left(p_{e}\right)\right)=p_{e} \in \mathbf{I}$, there is $J_{1}^{\prime} \in S \backslash\left\{I_{1}, I_{2}\right\}$ such that $I_{1} \leftarrow J_{1}^{\prime} \rightarrow I_{2}$. Therefore, from the definition of $T$, there is $J_{1} \in T \backslash\left\{I_{1}, I_{2}\right\}$ such that $I_{1} \leftarrow J_{1} \rightarrow I_{2}$.

Subcase (i). Suppose that $L_{2} \notin T$. Then by the definition of $T$ we have $L_{3} \in T$. Since $f(0) \notin \operatorname{Int}\left(L_{3}\right)$ and $L_{1}$ is $f$-covered by $J_{0} \in S \cap S^{\prime}$, from Remark 11.9 it follows that each $J \in T$ is $f$-covered by some basic interval of $T \backslash\{J\}$. By Lemma 14.1 we can assume that $J \nrightarrow J$ for each $J \in T$. In particular $I_{2} \nrightarrow I_{2}$ and so $I_{2} \rightarrow I_{1}$. Consider the loops $I_{1} \rightleftarrows I_{2}$ and $I_{1} \leftarrow J_{1} \rightarrow I_{2}$. Since $0 \notin I_{1}$ and $x_{0} \notin J_{1}$, from Lemma 11.5 and Proposition 7.2, we get that $B(k) \subset \operatorname{Per}(f)$. Hence the proposition holds.

Subcase (ii). Suppose that $L_{2} \in T$ and there are no basic intervals of $T \backslash\left\{L_{2}\right\}$ $f$-covering $L_{2}$. Consider $R=T \backslash\left\{L_{2}\right\}$. Since $f(0) \in \operatorname{Int}(A)$ and $L_{2} \rightarrow L_{2}$, $L_{2}$ does not $f$-cover $L_{1}$ and $L_{3}$. We claim that if $L_{2} \rightarrow M$ for some $M \in R$, then there is $J \in R \backslash\{M\}$ such that $J \rightarrow M$. Now we prove the claim. Notice that since $f(0) \in \operatorname{Int}(A)$, if $L_{2} \rightarrow M$, then $A \rightarrow M$ or $C \rightarrow M$. If $A \rightarrow M$, then take $J=L_{1}$ and we are done. So suppose that $C \rightarrow M$. If $L_{3} \in T$, then we take $J=L_{3}$ and we are done. Therefore suppose that $L_{3} \notin T$. From 
the facts that $f(0) \in \operatorname{Int}(A), f\left(f^{k-1}\left(p_{e}\right)\right)=p_{e} \in \mathbf{I}$ and by the continuity of $f$, it follows that each basic interval $N \in T, N \subset \mathbf{I}$ is $f$-covered by some basic interval of $S^{\prime}$ different from $C$. Hence we can assume that $M \nsubseteq \mathbf{I}$. Then $M \subset \mathbf{O}$. Since $C \rightarrow M, f(0) \in \operatorname{Int}(A), A \cup C \rightarrow A \cup C$, we have that $A \rightarrow\left[f(0), x_{a}\right] \subset A$ and $C \rightarrow[f(0), 0] \cup C \subset A \cup C$. By Lemma 11.12 we can suppose that there is $J_{2} \in S^{\prime}, J_{2} \neq C$ such that $J_{2} \rightarrow C$. Notice that $J_{2} \neq A$ because $f(A) \subset \mathbf{I}$. Moreover $J_{2} \neq B$; otherwise $L_{1}=A \cup B \rightarrow A \cup C=L_{2}$ in contradiction with the assumptions. Hence $J_{2} \in S \cap S^{\prime}$. Therefore either $J_{2} \rightarrow C \cup A=L_{2}$ or $J_{2} \rightarrow C \cup B=L_{3}$ a contradiction. So the claim is proved.

Thus each basic interval of $R$ is $f$-covered by some different basic interval of $R$. By the proof of Lemma 14.1, interchanching $T$ by $R$, we can assume that $N \nrightarrow N$ for each $N \in R$. In particular $I_{2} \nrightarrow I_{2}$ and so $I_{2} \rightarrow I_{1}$. Then we consider the paths $I_{1} \rightleftarrows I_{2}$ and $I_{1} \leftarrow J_{1} \rightarrow I_{2}$. Now the proposition follows as in Subcase (i) but thinking the proof of Lemma 11.5 with $R$ instead of $T$.

Subcase (iii). Suppose that $L_{2} \in T$ and $L_{2}$ is $f$-covered by some $M \in$ $T \backslash\left\{L_{2}\right\}$. Since $J_{0} \rightarrow L_{1}$ and $f(0) \notin \operatorname{Int}\left(L_{3}\right)$, from Remark 11.9 we have that each $J \in T$ is $f$-covered by some $N \in T \backslash\{J\}$. By Lemma 14.1 we can assume that $J \nrightarrow J$ for each $J \in T$. Then we get $I_{2} \rightarrow I_{1}$. Consider the paths $I_{1} \leftarrow J_{1} \rightarrow I_{2}, I_{1} \rightleftarrows I_{2}$, and the proposition follows as above.

Case 2. $f(0) \in \operatorname{Int}(B)$ and $x_{0} \in \mathbf{O}$. By definition of $x_{0}$ in Section 6 we have that there are no fixed points of $f$ in $B \cup C$. Therefore $B \rightarrow\left[f(0), x_{b}\right] \subset B$, $C \nrightarrow C$ and so $L_{3} \nrightarrow L_{3}$. Notice that $I_{1} \cap I_{2}=\left\{x_{0}\right\}$. If there are no basic intervals of $T f$-covering $I_{1} \cup I_{2}$, then we consider the $Y$ map

$$
g: \sigma \backslash \operatorname{Int}\left(I_{1} \cup I_{2}\right) \longrightarrow \sigma \backslash \operatorname{Int}\left(I_{1} \cup I_{2}\right)
$$

defined as $f$ restricted to $\sigma \backslash \operatorname{Int}\left(I_{1} \cup I_{2}\right)$. Clearly $k \in \operatorname{Per}(g)$. Hence by the $Y$ Theorem the result follows. So we can assume that there is $J_{1} \in T \backslash\left\{I_{1}, I_{2}\right\}$ such that $I_{1} \leftarrow J_{1} \rightarrow I_{2}$. We remark that $x_{0} \notin J_{1}$ and $0 \notin I_{1} \cup I_{2}$.

Subcase (i). Suppose that $L_{1} \notin T$. Then by definition of $T$ we have that $L_{2}, L_{3} \in T$. Since $L_{3} \nrightarrow L_{3}$ we get that $L_{3}$ is $f$-covered by some different basic interval of $T$. Moreover $f(0) \notin \operatorname{Int}\left(L_{2}\right)$, so by Remark 11.9 we have that each $J \in T$ is $f$-covered by some different basic interval of $T$. From Lemma 14.1 we can assume that $J \nrightarrow J$ for each $J \in T$. Then we obtain the loop $I_{1} \rightleftarrows I_{2}$. Therefore consider the above loop together with the path $I_{1} \leftarrow J_{1} \rightarrow I_{2}$. Since $x_{0} \notin J_{1}$ and $0 \notin I_{1}$ again the proposition follows as in the Subcase (i) of Case 1.

Subcase (ii). Suppose that $L_{1} \in T$ and there are no basic intervals of $T \backslash\left\{L_{1}\right\}$ $f$-covering $L_{1}$. Since $B \rightarrow\left[f(0), x_{b}\right] \subset B$, we get that $f\left(x_{a}\right) \in \mathbf{I}$ and $A \rightarrow$ $[f(0), 0] \cup A \subset B \cup A$. The facts that $f\left(x_{a}\right) \in \mathbf{I}, f(P \cap \mathbf{I}) \nsubseteq P \cap \mathbf{I}$ and the continuity of $f$ imply that there is $J_{0} \in S \cap S^{\prime}, J_{0} \subset \mathrm{I}$ such that $J_{0} \rightarrow A$. Moreover either $J_{0} \rightarrow A \cup B=L_{1}$ or $J_{0} \rightarrow A \cup C=L_{2}$. By the hypotheses we have that $J_{0} \rightarrow L_{2}$. Therefore $L_{2} \in T$ and $L_{2}$ is $f$-covered by $J_{0} \neq L_{2}$. Furthermore $L_{2} \nrightarrow L_{1}$ and so $C \nrightarrow\left[f(0), x_{b}\right] \subset B$. Since $C \nrightarrow C$, we get $C \rightarrow[f(0), 0] \cup A \subset B \cup A$. Then $L_{3}=B \cup C \rightarrow L_{1}$, and thus $L_{3} \notin T$. Set $R=T \backslash\left\{L_{1}\right\}=S \backslash\left\{L_{1}, L_{3}\right\}$. We claim that if $L_{1} \rightarrow M$ for some $M \in R$, then there is $J_{2} \in R, J_{2} \neq M$ such that $J_{2} \rightarrow M$. Notice that $J_{0} \rightarrow L_{2}$ and $J_{0} \in S \cap S^{\prime}$. Since $f(0) \in \operatorname{Int}(B)$ and $B=L_{1} \cap L_{3}$, then we can assume that $A \rightarrow M$ or $B \rightarrow M$. The facts that $f(0) \in \operatorname{Int}(B), f\left(f^{k-1}\left(p_{e}\right)\right)=p_{e} \in \mathbf{I}$ and the continuity of $f$ imply that if $M \subset \mathbf{I}$, then $M$ is $f$-covered by some 


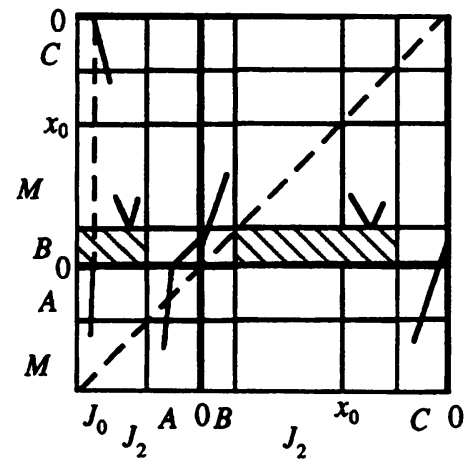

Figure 14.9. $M$ is $f$-covered by some $J_{2} \in R \backslash\{M\}$.

$J_{2} \in R, J_{2} \neq N$ (see Figure 14.9). So we can assume that $M \subset \mathbf{O}$. From the facts that $x_{b} \in P, L_{3} \notin T, L_{1}$ is not $f$-covered by any basic interval of $T \backslash\left\{L_{1}\right\}$ and by the continuity of $f$ it follows that there exists $J_{2} \in R$, $J_{2} \neq M$ such that $J_{2} \rightarrow M$ (see again Figure 14.9). Hence the claim is proved. Therefore each $J \in R$ is $f$-covered by some basic interval of $R \backslash\{J\}$. By the proof of Lemma 14.1, interchanging $T$ by $R$, we can assume that $J \nrightarrow J$ for each $J \in R$. Then we obtain $I_{1} \rightleftarrows I_{2}$. Consider the above loop and the path $I_{1} \leftarrow J_{1} \rightarrow I_{2}$. Now the proposition follows as the Subcase (ii) of Case 1.

Subcase (iii). Suppose that $L_{1} \in T$ and that $L_{1}$ is $f$-covered by some different basic interval of $T$. Since $L_{3} \nrightarrow L_{3}$ and $f(0) \notin \operatorname{Int}\left(L_{2}\right)$, from Remark 11.9 we have that each $J \in T$ is $f$-covered by some basic interval of $T \backslash\{J\}$. By Lemma 14.1 we can suppose that $J \nrightarrow J$ for each $J \in T$. Then we obtain $I_{1} \rightleftarrows I_{2}$. Consider the above loop together with the path $I_{1} \leftarrow J_{1} \rightarrow I_{2}$. This ends as in the Subcase (i).

\section{Proof of TheOREM 1.4(b)}

The goal of this section is to prove Theorem 1.4(b).

Lemma 15.1. Let $n_{s} \in \mathbb{N}_{s}, n_{g} \in \mathbb{N}_{g}$ and $n_{r} \in \mathbb{N}_{r}$. Denote by $a$ and $b$ two endpoints of $\mathbf{Y}$. Then there is an $\mathbf{Y}$ map $g$ having the branching point fixed such that $g(a)=a, g(b)=b$ and $\operatorname{Per}(g)=S\left(n_{s}\right) \cup G\left(n_{g}\right) \cup R\left(n_{r}\right)$.

Proof. Take $a_{1} \in(a, 0) \subset \mathbf{Y}$ and $b_{1} \in(b, 0) \subset \mathbf{Y}$. We consider the new topological space $\tilde{\mathbf{Y}}=\mathbf{Y} \backslash\left(\left[a, a_{1}\right) \cup\left[b, b_{1}\right)\right) \subset \mathbf{Y}$. Of course, $\tilde{\mathbf{Y}}$ is homeomorphic to $\mathbf{Y}$. Then by the $\mathbf{Y}$ Theorem there is an $\tilde{\mathbf{Y}}$ map $\tilde{g}$ having the branching point fixed and such that $\operatorname{Per}(\tilde{g})=S\left(n_{s}\right) \cup G\left(n_{g}\right) \cup R\left(n_{r}\right)$. Now we define an $Y$ map $g: \mathbf{Y} \longrightarrow \mathbf{Y}$ as follows. Let $g(x)=\tilde{g}(x)$ if $x \in \tilde{\mathbf{Y}}$. Let $g$ rectricted to $\left[a, a_{1}\right]$ be any homeomorphism between $\left[a, a_{1}\right]$ and $\left[a, \tilde{g}\left(a_{1}\right)\right]$ such that $g(a)=a$ and $g\left(a_{1}\right)=\tilde{g}\left(a_{1}\right)$. Finally, let $g$ restricted to $\left[b, b_{1}\right]$ be any homeomorphism between $\left[b, b_{1}\right]$ and $\left[b, \tilde{g}\left(b_{1}\right)\right]$ such that $g(b)=b$ and $g\left(b_{1}\right)=\tilde{g}\left(b_{1}\right)$.

By the construction of $g$ we get that $g$ has no periodic points of period $n>1$ in $\mathbf{Y} \backslash \tilde{\mathbf{Y}}$. Moreover, since $\left.g\right|_{\tilde{\mathbf{Y}}}=\tilde{g}$ it follows that $g(0)=0$ and 
$\operatorname{Per}(g)=\operatorname{Per}(\tilde{g})=S\left(n_{s}\right) \cup G\left(n_{g}\right) \cup R\left(n_{r}\right)$. Thus $g$ satisfies the statement of the lemma.

Proof of Theorem 1.4(b). Let $n_{s} \in \mathbb{N}_{s}, n_{g} \in \mathbb{N}_{g}, n_{r} \in \mathbb{N}_{r}$ and $n_{b} \in \mathbb{N}_{b}$, we need to construct a $\sigma$ map $f$ having the branching point fixed such that $\operatorname{Per}(f)=S\left(n_{s}\right) \cup G\left(n_{g}\right) \cup R\left(n_{r}\right) \cup B\left(n_{b}\right)$.

Since $Y$ is homeomorphic to $\{(x, y) \in \sigma: y \geq-1\} \subset \sigma$, in the rest of this proof we shall consider $\mathbf{Y}=\{(x, y) \in \sigma: y \geq-1\} \subset \sigma$. Let $U=(a, b)$ be the open subinterval of $\sigma$ defined as $U=\sigma \backslash \mathbf{Y} \subset \mathbf{O}$. Clearly $a$ and $b$ are two endpoints of $Y$. Let $g$ be the $Y$ map given by Lemma 15.1. We will to extend $g$ to a $\sigma$ map $f$ as follows. Let $f(x)=g(x)$ if $x \in \mathbf{Y}$. Then notice that $a, b$ and 0 are fixed points for $f$. Now we need to define $\left.f\right|_{U}$.

Set $n=n_{b}$. Let $Q=\left\{q_{1}, q_{2}, \cdots, q_{n}\right\} \subset U$ be such that $q_{i} \neq q_{j}$ for $i \neq j,\left(a, q_{1}\right) \cap Q=\varnothing,\left(q_{n}, b\right) \cap Q=\varnothing$ and for each $i \in\{1,2, \cdots, n-$ 1\} $\left(q_{i}, q_{i+1}\right) \cap Q=\varnothing$. Define $f\left(q_{i}\right)=q_{i+1}$ for $i \in\{1,2, \cdots, n-1\}$ and $f\left(q_{n}\right)=q_{1}$. For each $i \in\{1,2, \cdots, n-2\}$ let $f$ restricted to $\left[q_{i}, q_{i+1}\right]$ be any homeomorphism between $\left[q_{i}, q_{i+1}\right]$ and $\left[q_{i+1}, q_{i+2}\right]$. Let $f$ restricted to $\left[q_{n-1}, q_{n}\right]$ be any homeomorphism between $\left[q_{n-1}, q_{n}\right]$ and $\left[q_{n}, q_{1}\right]$. Let $f$ restricted to $\left[q_{n}, b\right]$ be any homeomorphism between $\left[q_{n}, b\right]$ and $\left[b, q_{1}\right]$. Finally let $f$ restricted to $\left[a, q_{1}\right]$ be any homeomorphism between $\left[a, q_{1}\right]$ and $\left[a, q_{2}\right]$.

Since $f(0)=0, f$ is a $\sigma$ map. Of course $Q$ is a periodic orbit of period $n$ for $f$. Consider the set of basic intervals associated to $Q \cup\{a\}$. Therefore we obtain a non-repetitive loop $\left[a, q_{1}\right] \rightarrow\left[q_{1}, q_{2}\right] \rightarrow\left[q_{2}, q_{3}\right] \rightarrow \cdots \rightarrow\left[q_{n-1}, q_{n}\right] \rightarrow$ $\left[a, q_{1}\right] \rightarrow\left[a, q_{1}\right] \rightarrow \cdots \rightarrow\left[a, q_{1}\right]$ of length $m$ for each $m \geq n=n_{b}$. By Lemma 3.3 there exists a fixed point $x \in\left[q_{1}, q_{2}\right]$ of $f^{m}$ for each $m \geq n_{b}$. By the definition of $f x$ has period $m$. So $B\left(n_{b}\right) \subset \operatorname{Per}(f)$. By construction of $f$ all periodic points of $f$ on $U$ have period at least $n_{b}$. Since $\left.f\right|_{\mathbf{Y}}=g$, the set of periods of periodic point of $\left.f\right|_{\mathrm{Y}}$ is $S\left(n_{s}\right) \cup G\left(n_{g}\right) \cup R\left(n_{r}\right)$. Consequently $\operatorname{Per}(f)=S\left(n_{s}\right) \cup G\left(n_{g}\right) \cup R\left(n_{r}\right) \cup B\left(n_{b}\right)$ and we are done.

\section{REFERENCES}

[ALM1] L. Alsedà, J. Llibre and M. Misiurewicz, Periodic orbits of maps of $\mathbf{Y}$, Trans. Amer. Math. Soc. 313 (1989), 475-538.

[ALM2] C_ Combinatorial dynamics in dimension one, Advanced Series in Nonlinear Dynamics, Vol. 5, World Scientific, 1993.

[Ba] S. Baldwin, An extension of Sarkovskii's Theorem to the n-od, Ergodic Theory Dynamical Systems 11 (1991), 249-271.

[BI] L. Block, Periods of periodic points of maps of the circle which have a fixed point, Proc. Amer. Math. Soc. 82 (1981), 481-486.

[BGMY] L. Block, J. Guckenheimer, M. Misiurewicz and L. S. Young, Periodic points and topological entropy of one dimensional maps, Lecture Notes in Math., vol. 819, Springer-Verlag, Heidelberg, 1980, pp. 18-34.

[LPR] J. Llibre, J. Paraños and A. Rodriguez, Sets of periods for maps on connected graphs with zero Euler characteristic having all branching ponits fixed, Preprint.

[P] X. Paraños, Estructura periodica de aplicacions continuas dun grafo non contráctil, Thesis, Universidade de Santiago de Compostela, 1993.

[R] W. Rudin, Principles of mathematical analysis, McGraw-Hill Internat. Eds. Math. Ser., McGraw-Hill, 1987. 
[Sa] A. N. Sarkovskii, Co-existence of the cycles of a continuous mapping of the line into itself, Ukrain. Math. Zh. 16 (1964), 61-71. (Russian)

[St] P. D. Straffin, Periodic points of continuous functions, Math. Mag. 51 (1978), 99-105.

Departament de Matemàtica Aplicada II, Universitat Politecnica de Catalunya, 08222 Terrassa, Barcelona, Spain

E-mail address: leseduarte@ma2.upc.es

Departament de Matemàtiques, facultat de Ciencies, Universitat autònoma de Barcelona, 08193 Bellaterra, Barcelona, Spain

$E$-mail address: imat0ecc.uab.es 